quatrième série-tome $46 \quad$ fascicule 2 mars-avril 2013

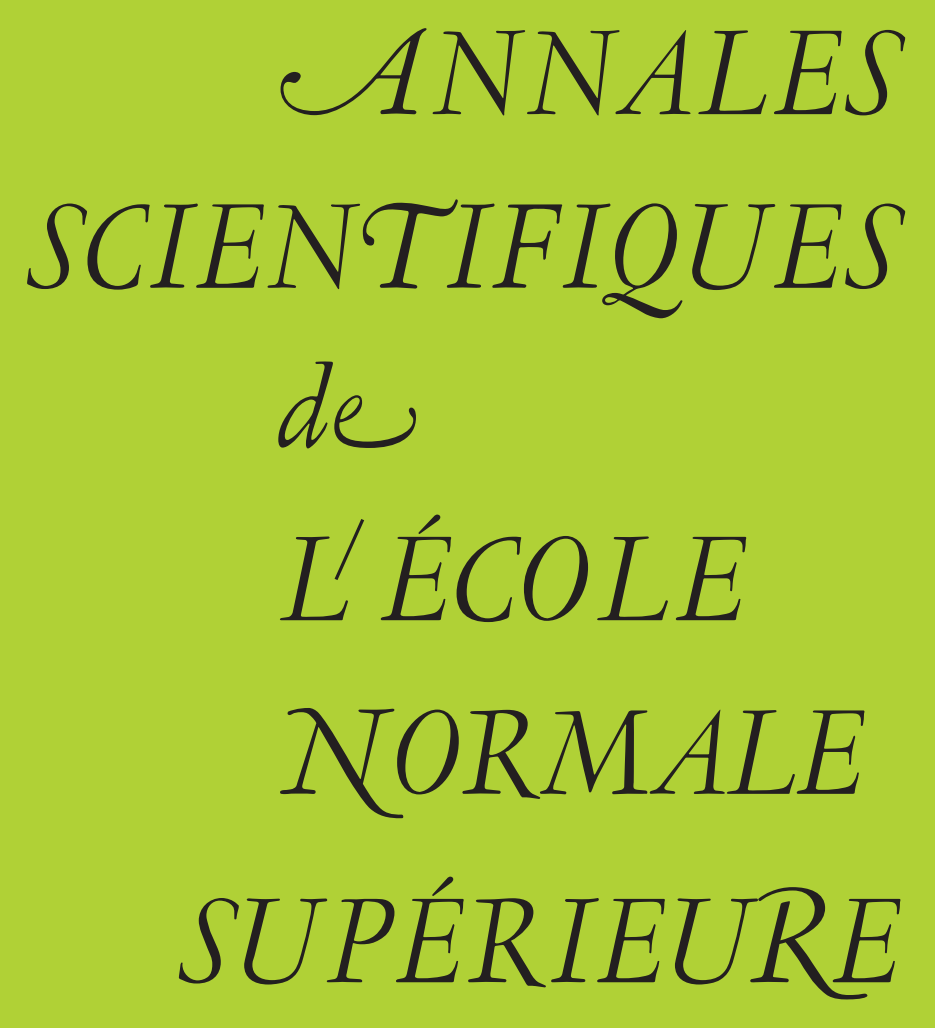

Massimiliano BERTI \& Luca BIASCO \& Michela PROCESI $K A M$ theory for the Hamiltonian derivative wave equation 


\title{
KAM THEORY FOR THE HAMILTONIAN DERIVATIVE WAVE EQUATION
}

\author{
BY Massimiliano BERTI, Luca BIASCO And Michela PROCESI
}

\begin{abstract}
We prove an infinite dimensional KAM theorem which implies the existence of Cantor families of small-amplitude, reducible, elliptic, analytic, invariant tori of Hamiltonian derivative wave equations.

RÉSUMÉ. - Nous prouvons un théorème KAM en dimension infinie, qui implique l'existence de familles de Cantor de tores invariants de petite amplitude, réductibles, elliptiques et analytiques, pour les équations des ondes hamiltoniennes avec dérivées.
\end{abstract}

\section{Introduction}

In the last years many progresses have been done concerning KAM theory for nonlinear Hamiltonian PDEs. The first existence results were given by Kuksin [20] and Wayne [32] for semilinear wave (NLW) and Schrödinger equations (NLS) in one space dimension (1d) under Dirichlet boundary conditions, see [27]-[28] and [23] for further developments. The approach of these papers consists in generating iteratively a sequence of symplectic changes of variables which bring the Hamiltonian into a constant coefficients (=reducible) normal form with an elliptic (=linearly stable) invariant torus at the origin. Such a torus is filled by quasiperiodic solutions with zero Lyapunov exponents. This procedure requires to solve, at each step, constant-coefficients linear "homological equations" by imposing the "second order Melnikov" non-resonance conditions. Unfortunately these (infinitely many) conditions are violated already for periodic boundary conditions.

In this case, existence of quasi-periodic solutions for semilinear $1 d$-NLW and NLS equations, was first proved by Bourgain [5] by extending the Newton approach introduced by Craig-Wayne [11] for periodic solutions. Its main advantage is to require only the "first order Melnikov" non-resonance conditions (the minimal assumptions) for solving the homological equations. Actually, developing this perspective, Bourgain was also able to prove in [6], [8] the existence of quasi-periodic solutions for NLW and NLS (with Fourier multipliers) in higher space dimensions; see also the recent extensions in [4], [3], [31]. The main drawback 
of this approach is that the homological equations are linear PDEs with non-constant coefficients. Translated in the KAM language this implies a non-reducible normal form around the torus and then a lack of informations about the stability of the quasi-periodic solutions.

Later on, existence of reducible elliptic tori was proved by Chierchia-You [9] for semilinear $1 d$-NLW, and, more recently, by Eliasson-Kuksin [14] for NLS (with Fourier multipliers) in any space dimension; see also Procesi-Xu [30], Geng-Xu-You [15].

An important problem concerns the study of PDEs where the nonlinearity involves derivatives. A comprehension of this situation is of major importance since most of the models coming from Physics are of this kind.

In this direction KAM theory has been extended to deal with $\mathrm{KdV}$ equations by Kuksin [21]-[22], Kappeler-Pöschel [19], and, for the 1d-derivative NLS (DNLS) and BenjiaminOno equations, by Liu-Yuan [24]. The key idea of these results is again to provide only a non-reducible normal form around the torus. However, in this case, the homological equations with non-constant coefficients are only scalar (not an infinite system as in the Craig-Wayne-Bourgain approach). We remark that the KAM proof is more delicate for DNLS and Benjiamin-Ono, because these equations are less "dispersive" than KdV, i.e., the eigenvalues of the principal part of the differential operator grow only quadratically at infinity, and not cubically as for $\mathrm{KdV}$. As a consequence of this difficulty, the quasi-periodic solutions in [21], [19] are analytic, in [24], only $C^{\infty}$. Actually, for the applicability of these KAM schemes, the more dispersive the equation is, the more derivatives in the nonlinearity can be supported. The limit case of the derivative nonlinear wave equation (DNLW) - which is not dispersive at all — is excluded by these approaches.

In the paper [5] (which proves the existence of quasi-periodic solutions for semilinear $1 d$-NLS and NLW), Bourgain claims, in the last remark, that his analysis works also for the Hamiltonian "derivation" wave equation

$$
y_{t t}-y_{x x}+g(x) y=\left(-\frac{d^{2}}{d x^{2}}\right)^{1 / 2} F(x, y)
$$

see also [7], page 81. Unfortunately no details are given. However, Bourgain [7] provided a detailed proof of the existence of periodic solutions for the non-Hamiltonian equation

$$
y_{t t}-y_{x x}+\mathrm{m} y+y_{t}^{2}=0, \quad \mathrm{~m} \neq 0 .
$$

These kinds of problems have been then reconsidered by Craig in [10] for more general Hamiltonian derivative wave equations like

$$
y_{t t}-y_{x x}+g(x) y+f\left(x, D^{\beta} y\right)=0, \quad x \in \mathbb{T},
$$

where $g(x) \geq 0$ and $D$ is the first order pseudo-differential operator $D:=\sqrt{-\partial_{x x}+g(x)}$. The perturbative analysis of Craig-Wayne [11] for the search of periodic solutions works when $\beta<1$. The main reason is that the wave equation vector field gains one derivative and then the nonlinear term $f\left(D^{\beta} u\right)$ has a strictly weaker effect on the dynamics for $\beta<1$. The case $\beta=1$ is left as an open problem. Actually, in this case, the small divisors problem for periodic solutions has the same level of difficulty of quasi-periodic solutions with 2 frequencies. 
The goal of this paper is to extend KAM theory to deal with the Hamiltonian derivative wave equation

$$
y_{t t}-y_{x x}+\mathrm{m} y+f(D y)=0, \quad \mathrm{~m}>0, \quad D:=\sqrt{-\partial_{x x}+\mathrm{m}}, \quad x \in \mathbb{T},
$$

with real analytic nonlinearities (see Remark 7.1)

$$
f(s)=a s^{3}+\sum_{k \geq 5} f_{k} s^{k}, \quad a \neq 0 .
$$

We write Equation (1.1) as the infinite dimensional Hamiltonian system

$$
u_{t}=-\mathrm{i} \partial_{\bar{u}} H, \quad \bar{u}_{t}=\mathrm{i} \partial_{u} H
$$

with Hamiltonian

$$
H(u, \bar{u}):=\int_{\mathbb{T}} \bar{u} D u+F\left(\frac{u+\bar{u}}{\sqrt{2}}\right) d x, \quad F(s):=\int_{0}^{s} f,
$$

in the complex unknown

$$
u:=\frac{1}{\sqrt{2}}\left(D y+\mathrm{i} y_{t}\right), \quad \bar{u}:=\frac{1}{\sqrt{2}}\left(D y-\mathrm{i} y_{t}\right), \quad \mathrm{i}:=\sqrt{-1} .
$$

Setting $u=\sum_{j \in \mathbb{Z}} u_{j} e^{\mathrm{i} j x}$ (similarly for $\bar{u}$ ), we obtain the Hamiltonian in infinitely many coordinates

$$
H=\sum_{j \in \mathbb{Z}} \lambda_{j} u_{j} \bar{u}_{j}+\int_{\mathbb{T}} F\left(\frac{1}{\sqrt{2}} \sum_{j \in \mathbb{Z}}\left(u_{j} e^{\mathrm{i} j x}+\bar{u}_{j} e^{-\mathrm{i} j x}\right)\right) d x
$$

where

$$
\lambda_{j}:=\sqrt{j^{2}+\mathrm{m}}
$$

are the eigenvalues of the diagonal operator $D$. Note that the nonlinearity in (1.1) is $x$-independent implying, for (1.3), the conservation of the momentum $-\mathrm{i} \int_{\mathbb{T}} \bar{u} \partial_{x} u d x$. This symmetry allows to simplify somehow the KAM proof (a similar idea was used by Geng-You [16]).

For every choice of the tangential sites $\mathcal{I}:=\left\{j_{1}, \ldots, j_{n}\right\} \subset \mathbb{Z}, n \geq 2$, the integrable Hamiltonian $\sum_{j \in \mathbb{Z}} \lambda_{j} u_{j} \bar{u}_{j}$ has the invariant tori $\left\{u_{j} \bar{u}_{j}=\xi_{j}\right.$, for $j \in \mathcal{I}, u_{j}=\bar{u}_{j}=0$ for $j \notin \mathcal{I}\}$ parametrized by the actions $\xi=\left(\xi_{j}\right)_{j \in \mathcal{I}} \in \mathbb{R}^{n}$. The next KAM result states the existence of nearby invariant tori for the complete Hamiltonian $H$ in (1.4).

Theorem 1.1. - The Equation (1.1)-(1.2) admits Cantor families of small-amplitude, analytic, quasi-periodic solutions with zero Lyapunov exponents and whose linearized equation is reducible to constant coefficients. Such Cantor families have asymptotically full measure at the origin in the set of parameters.

The proof of Theorem 1.1 is based on the abstract infinite dimensional KAM Theorem 4.1, which provides a reducible normal form (see (4.12)) around the elliptic invariant torus, and on the measure estimates Theorem 4.2. The key point in proving Theorem 4.2 is the asymptotic bound (4.9) on the perturbed normal frequencies $\Omega^{\infty}(\xi)$ after the KAM iteration. This allows to prove that the second order Melnikov non-resonance conditions (4.11) are fulfilled for an asymptotically full measure set of parameters (see (4.16)). The estimate (4.9), in turn, is achieved by exploiting the quasi-Töplitz property of the perturbation. This notion has been introduced by Procesi-Xu [30] in the context of NLS in higher space 
dimensions and it is similar, in spirit, to the Töplitz-Lipschitz property in Eliasson-Kuksin [14]. The precise formulation of quasi-Töplitz functions, adapted to the DNLW setting, is given in Definition 3.4 below.

Let us roughly explain the main ideas and techniques for proving Theorems 4.1, 4.2. These theorems concern, as usual, a parameter dependent family of analytic Hamiltonians of the form

$$
H=\omega(\xi) \cdot y+\Omega(\xi) \cdot z \bar{z}+P(x, y, z, \bar{z} ; \xi)
$$

where $(x, y) \in \mathbb{T}^{n} \times \mathbb{R}^{n}, z, \bar{z}$ are infinitely many variables, $\omega(\xi) \in \mathbb{R}^{n}, \Omega(\xi) \in \mathbb{R}^{\infty}$ and $\xi \in \mathbb{R}^{n}$. The frequencies $\Omega_{j}(\xi)$ are close to the unperturbed frequencies $\lambda_{j}$ in (1.5).

As is well known, the main difficulty of the KAM iteration which provides a reducible KAM normal form like (4.12) is to fulfill, at each iterative step, the second order Melnikov non-resonance conditions. Actually, following the formulation of the KAM theorem given in [2], it is sufficient to verify

$$
\left|\omega^{\infty}(\xi) \cdot k+\Omega_{i}^{\infty}(\xi)-\Omega_{j}^{\infty}(\xi)\right| \geq \frac{\gamma}{1+|k|^{\tau}}, \quad \gamma>0,
$$

only for the "final" frequencies $\omega^{\infty}(\xi)$ and $\Omega^{\infty}(\xi)$, see (4.11), and not along the inductive iteration.

The application of the usual KAM theory (see e.g., [20], [27]-[28]), to the DNLW equation provides only the asymptotic decay estimate

$$
\Omega_{j}^{\infty}(\xi)=j+O(1) \text { for } j \rightarrow+\infty .
$$

Such a bound is not enough: the set of parameters $\xi$ satisfying (1.7) could be empty. Note that for the semilinear NLW equation (see e.g., [27]) the frequencies decay asymptotically faster, namely like $\Omega_{j}^{\infty}(\xi)=j+O(1 / j)$.

The key idea for verifying the second order Melnikov non-resonance conditions (1.7) for DNLW is to prove the higher order asymptotic decay estimate (see (4.9), (4.2))

$$
\Omega_{j}^{\infty}(\xi)=j+a_{+}(\xi)+\frac{\mathrm{m}}{2 j}+O\left(\frac{\gamma^{2 / 3}}{j}\right) \quad \text { for } \quad j \geq O\left(\gamma^{-1 / 3}\right)
$$

where $a_{+}(\xi)$ is a constant independent of $j$ (an analogous expansion holds for $j \rightarrow-\infty$ with a possibly different limit constant $a_{-}(\xi)$ ). In this way infinitely many conditions in (1.7) are verified by imposing only first order Melnikov conditions like $\left|\omega^{\infty}(\xi) \cdot k+h\right| \geq 2 \gamma^{2 / 3} /|k|^{\tau}$, $h \in \mathbb{Z}$. Indeed, for $i>j>O\left(|k|^{\tau} \gamma^{-1 / 3}\right)$, we get

$$
\begin{aligned}
\left|\omega^{\infty}(\xi) \cdot k+\Omega_{i}^{\infty}(\xi)-\Omega_{j}^{\infty}(\xi)\right| & =\left|\omega^{\infty}(\xi) \cdot k+i-j+\frac{\mathrm{m}(i-j)}{2 i j}+O\left(\gamma^{2 / 3} / j\right)\right| \\
& \geq 2 \gamma^{2 / 3}|k|^{-\tau}-O\left(|k| / j^{2}\right)-O\left(\gamma^{2 / 3} / j\right) \geq \gamma^{2 / 3}|k|^{-\tau}
\end{aligned}
$$

noting that $i-j$ is integer and $|i-j|=O(|k|)$ (otherwise no small divisors occur). We refer to Section 6 for the precise arguments, see in particular Lemma 6.2.

The asymptotic decay (4.9) for the perturbed frequencies $\Omega^{\infty}(\xi)$ is achieved thanks to the "quasi-Töplitz" property of the perturbation (Definition 3.4). Let us roughly explain this 
notion. The new normal frequencies after each KAM step are $\Omega_{j}^{+}=\Omega_{j}+P_{j}^{0}$ where the corrections $P_{j}^{0}$ are the coefficients of the quadratic form

$$
P^{0} z \bar{z}:=\sum_{j} P_{j}^{0} z_{j} \bar{z}_{j}, \quad P_{j}^{0}:=\int_{\mathbb{T}^{n}}\left(\partial_{z_{j} \bar{z}_{j}}^{2} P\right)(x, 0,0,0 ; \xi) d x .
$$

We say that a quadratic form $P^{0}$ is quasi-Töplitz if it has the form

$$
P^{0}=T+R
$$

where $T$ is a Töplitz matrix (i.e., constant on the diagonals) and $R$ is a "small" remainder satisfying $R_{j j}=O(1 / j)$ (see Lemma 5.2). Then (1.9) follows with $a:=T_{j j}$ which is independent of $j$. The rate of decay $O(1 / j)$ is the natural one for the application to the DNLW equation, due to the asymptotic expansion $\sqrt{j^{2}+\mathrm{m}}=j+\mathrm{m} /(2 j)+O\left(1 / j^{3}\right)$ as $j \rightarrow+\infty$, see (5.12). We expect that the class of quasi-Töplitz functions defined with a weaker decay, say $O\left(1 /|j|^{\beta}\right), \beta>0$, would still be closed under Poisson brackets; see below.

Since the quadratic perturbation $P^{0}$ along the KAM iteration does not depend only on the quadratic perturbation at the previous steps, we need to extend the notion of quasi-Töplitz to general (non-quadratic) analytic functions. The preservation of the quasi-Töplitz property of the perturbations $P$ at each KAM step (with just slightly modified parameters) holds in view of the following key facts:

1. the Poisson bracket of two quasi-Töplitz functions is quasi-Töplitz (Proposition 3.1),

2. the hamiltonian flow generated by a quasi-Töplitz function preserves the quasi-Töplitz property (Proposition 3.2),

3. the solution of the homological equation with a quasi-Töplitz perturbation is quasiTöplitz (Proposition 5.1).

We note that, in [14], the analogous property 1 (and therefore 2) for Töplitz-Lipschitz functions is proved only when one of them is quadratic.

The definition of quasi-Töplitz functions heavily relies on properties of projections. However, for an analytic function in infinitely many variables, such projections may not be well defined unless the Taylor-Fourier series (see (2.28)) is absolutely convergent. For such reason, instead of the sup-norm, we use the majorant norm (see (2.12), (2.54)), for which the bounds (2.14) and (2.55) on projections hold (see also Remark 2.4).

We underline that the majorant norm of a vector field introduced in (2.54) is very different from the weighted norm introduced by Pöschel in [26]-Appendix C, which works only in finite dimension, see comments in [26] after Lemma C.2 and Remark 2.3. In Section 2 we show its properties, in particular the key estimate of the majorant norm of the commutator of two vector fields (see Lemma 2.15). A related majorant norm for functions and vector fields is introduced in Bambusi-Grébert [1] in a context of Sobolev spaces (and with tame modulus properties); see also [25].

Before concluding this introduction we also mention the recent KAM theorem of Grébert-Thomann [18] for the quantum harmonic oscillator with semilinear nonlinearity. Also here the eigenvalues grow to infinity only linearly. We quote the normal form results of Delort-Szeftel [13], Delort [12], for quasi-linear wave equations, where only finitely many steps of normal form can be performed. Finally we also mention the recent work by Gérard-Grellier [17] on Birkhoff normal form for a degenerate "half-wave" equation. 
The paper is organized as follows:

- In Section 2 we define the majorant norm of formal power series of scalar functions (Definition 2.2) and vector fields (Definition 2.6) and we investigate the relations with the notion of analiticity, see Lemmata 2.1, 2.2, 2.3, 2.11 and Corollary 2.1. Then we prove Lemma 2.15 on commutators.

- In Section 3 we define the Töplitz (Definition 3.3) and Quasi-Töplitz functions (Definition 3.4). Then we prove that this class of functions is closed under Poisson brackets (Proposition 3.1) and composition with the Hamiltonian flow (Proposition 3.2).

- In Section 4 we state the abstract KAM Theorem 4.1. The first part of Theorem 4.1 follows by the KAM Theorem 5.1 in [2]. The main novelty is part II, in particular the asymptotic estimate (4.9) of the normal frequencies.

- In Section 5 we prove the abstract KAM Theorem 4.1.

We first perform (as in Theorem 5.1 in [2]) a first normal form step, which makes Theorem 4.1 suitable for the direct application to the wave equation.

In Proposition 5.1 we prove that the solution of the homological equation with a quasi-Töplitz perturbation is quasi-Töplitz. Then the main results of the KAM step concern the asymptotic estimates of the perturbed frequencies (Section 5.2.3) and the Töplitz estimates of the new perturbation (Section 5.2.4).

- In Section 6 we prove Theorem 4.2: the second order Melnikov non-resonance conditions are fulfilled for a set of parameters with large measure, see (4.16). We use the conservation of momentum to avoid the presence of double eigenvalues.

- In Section 7 we finally apply the abstract KAM Theorem 4.1 to the DNLW Equation (1.1)-(1.2), proving Theorem 1.1. We first verify that the Hamiltonian (1.4) is quasi-Töplitz (Lemma 7.1), as well as the Birkhoff normal form Hamiltonian (7.8) of Proposition 7.1. The main technical difficulties concern the proof in Lemma 7.4 that the generating function (7.17) of the Birkhoff symplectic transformation is also quasi-Töplitz (and the small divisors Lemma 7.2). In Section 7.2 we prove that the perturbation, obtained after the introduction of the action-angle variables, is still quasi-Töplitz (Proposition 7.2). Finally in Section 7.3 we prove Theorem 1.1 applying Theorems 4.1 and 4.2.

\section{Functional setting}

Given a finite subset $\mathcal{I} \subset \mathbb{Z}$ (possibly empty), $a \geq 0, p>1 / 2$, we define the Hilbert space

$$
\ell_{\mathcal{I}}^{a, p}:=\left\{z=\left\{z_{j}\right\}_{j \in \mathbb{Z} \backslash \mathcal{I}}, z_{j} \in \mathbb{C}:\|z\|_{a, p}^{2}:=\sum_{j \in \mathbb{Z} \backslash \mathcal{I}}\left|z_{j}\right|^{2} e^{2 a|j|}\langle j\rangle^{2 p}<\infty\right\} .
$$

When $\mathcal{I}=\varnothing$ we denote $\ell^{a, p}:=\ell_{\mathcal{I}}^{a, p}$. We consider the direct product

$$
E:=\mathbb{C}^{n} \times \mathbb{C}^{n} \times \ell_{\mathcal{I}}^{a, p} \times \ell_{\mathcal{I}}^{a, p}
$$

where $n$ is the cardinality of $\mathcal{I}$. We endow the space $E$ with the $(s, r)$-weighted norm

$$
v=(x, y, z, \bar{z}) \in E, \quad\|v\|_{E}:=\|v\|_{E, s, r}=\frac{|x|_{\infty}}{s}+\frac{|y|_{1}}{r^{2}}+\frac{\|z\|_{a, p}}{r}+\frac{\|\bar{z}\|_{a, p}}{r}
$$


where, $0<s, r<1$, and $|x|_{\infty}:=\max _{h=1, \ldots, n}\left|x_{h}\right|,|y|_{1}:=\sum_{h=1}^{n}\left|y_{h}\right|$. Note that, for all $s^{\prime} \leq s, r^{\prime} \leq r$,

$$
\|v\|_{E, s^{\prime}, r^{\prime}} \leq \max \left\{s / s^{\prime},\left(r / r^{\prime}\right)^{2}\right\}\|v\|_{E, s, r} .
$$

We shall also use the notations

$$
z_{j}^{+}=z_{j}, \quad z_{j}^{-}=\bar{z}_{j} .
$$

We identify a vector $v \in E$ with the sequence $\left\{v^{(j)}\right\}_{j \in \mathcal{J}}$ with indices in

$$
\mathcal{J}:=\left\{j=\left(j_{1}, j_{2}\right), j_{1} \in\{1,2,3,4\}, j_{2} \in\left\{\begin{array}{ll}
\{1, \ldots, n\} & \text { if } j_{1}=1,2 \\
\mathbb{Z} \backslash \mathcal{I} & \text { if } j_{1}=3,4
\end{array}\right\}\right.
$$

and components

$$
v^{\left(1, j_{2}\right)}:=x_{j_{2}}, v^{\left(2, j_{2}\right)}:=y_{j_{2}}\left(1 \leq j_{2} \leq n\right), v^{\left(3, j_{2}\right)}:=z_{j_{2}}, v^{\left(4, j_{2}\right)}:=\bar{z}_{j_{2}}\left(j_{2} \in \mathbb{Z} \backslash \mathcal{I}\right),
$$

more compactly

$$
v^{(1, \cdot)}:=x, v^{(2, \cdot)}:=y,, v^{(3, \cdot)}:=z,, v^{(4, \cdot)}:=\bar{z} .
$$

We denote by $\left\{e_{j}\right\}_{j \in \mathcal{J}}$ the orthogonal basis of the Hilbert space $E$, where $e_{j}$ is the sequence with all zeros, except the $j_{2}$-th entry of its $j_{1}$-th components, which is 1 . Then every $v \in E$ writes $v=\sum_{j \in \mathcal{J}} v^{(j)} e_{j}, v^{(j)} \in \mathbb{C}$. We also define the toroidal domain

$$
D(s, r):=\mathbb{T}_{s}^{n} \times D(r):=\mathbb{T}_{s}^{n} \times B_{r^{2}} \times B_{r} \times B_{r} \subset E
$$

where $D(r):=B_{r^{2}} \times B_{r} \times B_{r}$,

$$
\mathbb{T}_{s}^{n}:=\left\{x \in \mathbb{C}^{n}: \max _{h=1, \ldots, n}\left|\operatorname{Im} x_{h}\right|<s\right\}, B_{r^{2}}:=\left\{y \in \mathbb{C}^{n}:|y|_{1}<r^{2}\right\}
$$

and $B_{r} \subset \ell_{\mathcal{I}}^{a, p}$ is the open ball of radius $r$ centered at zero. We think $\mathbb{T}^{n}$ as the $n$-dimensional torus $\mathbb{T}^{n}:=2 \pi \mathbb{R}^{n} / \mathbb{Z}^{n}$, namely $f: D(s, r) \rightarrow \mathbb{C}$ means that $f$ is $2 \pi$-periodic in each $x_{h}$-variable, $h=1, \ldots, n$.

REMARK 2.1. - If $n=0$ then $D(s, r) \equiv B_{r} \times B_{r} \subset \ell^{a, p} \times \ell^{a, p}$.

\subsection{Majorant norm}

2.1.1. Scalar functions. - We consider formal power series with infinitely many variables

$$
f(v)=f(x, y, z, \bar{z})=\sum_{(k, i, \alpha, \beta) \in \mathbb{I}} f_{k, i, \alpha, \beta} e^{\mathrm{i} k \cdot x} y^{i} z^{\alpha} \bar{z}^{\beta}
$$

with coefficients $f_{k, i, \alpha, \beta} \in \mathbb{C}$ and multi-indices in

$$
\mathbb{I}:=\mathbb{Z}^{n} \times \mathbb{N}^{n} \times \mathbb{N}^{(\mathbb{Z} \backslash \mathcal{I})} \times \mathbb{N}^{(\mathbb{Z} \backslash \mathcal{I})}
$$

where

$$
\mathbb{N}^{(\mathbb{Z} \backslash \mathcal{I})}:=\left\{\alpha:=\left(\alpha_{j}\right)_{j \in \mathbb{Z} \backslash \mathcal{I}} \in \mathbb{N}^{\mathbb{Z}} \text { with }|\alpha|:=\sum_{j \in \mathbb{Z} \backslash \mathcal{I}} \alpha_{j}<+\infty\right\} .
$$

In (2.7) we use the standard multi-indices notation $z^{\alpha} \bar{z}^{\beta}:=\Pi_{j \in \mathbb{Z} \backslash \mathcal{I}} z_{j}^{\alpha_{j}} \bar{z}_{j}^{\beta_{j}}$. We denote the monomials

$$
\mathfrak{m}_{k, i, \alpha, \beta}(v)=\mathfrak{m}_{k, i, \alpha, \beta}(x, y, z, \bar{z}):=e^{\mathrm{i} k \cdot x} y^{i} z^{\alpha} \bar{z}^{\beta}
$$


REMARK 2.2. - If $n=0$ the set II reduces to $\mathbb{N}^{\mathbb{Z}} \times \mathbb{N}^{\mathbb{Z}}$ and the formal series to $f(z, \bar{z})=$ $\sum_{(\alpha, \beta) \in \mathbb{I}} f_{\alpha, \beta} z^{\alpha} \bar{z}^{\beta}$.

We define the "majorant" of $f$ as

$$
(M f)(v)=(M f)(x, y, z, \bar{z}):=\sum_{(k, i, \alpha, \beta) \in \mathbb{I}}\left|f_{k, i, \alpha, \beta}\right| e^{\mathrm{i} k \cdot x} y^{i} z^{\alpha} \bar{z}^{\beta} .
$$

We now discuss the convergence of formal series.

Definition 2.1. - A series

$$
\sum_{(k, i, \alpha, \beta) \in \mathbb{I}} c_{k, i, \alpha, \beta}, \quad c_{k, i, \alpha, \beta} \in \mathbb{C},
$$

is absolutely convergent if the function $\mathbb{I} \ni(k, i, \alpha, \beta) \mapsto c_{k, i, \alpha, \beta} \in \mathbb{C}$ is in $L^{1}(\mathbb{I}, \mu)$ where $\mu$ is the counting measure of $\mathbb{I}$. Then we set

$$
\sum_{(k, i, \alpha, \beta) \in \mathbb{I}} c_{k, i, \alpha, \beta}:=\int_{\mathbb{I}} c_{k, i, \alpha, \beta} d \mu .
$$

By the properties of the Lebesgue integral, given any sequence $\left\{I_{l}\right\}_{l \geq 0}$ of finite subsets $I_{l} \subset \mathbb{I}$ with $I_{l} \subset I_{l+1}$ and $\cup_{l \geq 0} I_{l}=\mathbb{I}$, the absolutely convergent series

$$
\sum_{k, i, \alpha, \beta} c_{k, i, \alpha, \beta}:=\sum_{(k, i, \alpha, \beta) \in \mathbb{I}} c_{k, i, \alpha, \beta}=\lim _{l \rightarrow \infty} \sum_{(k, i, \alpha, \beta) \in I_{l}} c_{k, i, \alpha, \beta} .
$$

Definition 2.2 (Majorant-norm: scalar functions). - The majorant-norm of a formal power series (2.7) is

$$
\|f\|_{s, r}:=\sup _{(y, z, \bar{z}) \in D(r)} \sum_{k, i, \alpha, \beta}\left|f_{k, i, \alpha, \beta}\right| e^{|k| s}\left|y^{i}\right|\left|z^{\alpha}\right|\left|\bar{z}^{\beta}\right|
$$

where $|k|:=|k|_{1}:=\left|k_{1}\right|+\cdots+\left|k_{n}\right|$.

By (2.7) and (2.12) we clearly have $\|f\|_{s, r}=\|M f\|_{s, r}$.

For every subset of indices $I \subset \mathbb{I}$, we define the projection

$$
\left(\Pi_{I} f\right)(x, y, z, \bar{z}):=\sum_{(k, i, \alpha, \beta) \in I} f_{k, i, \alpha, \beta} e^{\mathrm{i} k \cdot x} y^{i} z^{\alpha} \bar{z}^{\beta}
$$

of the formal power series $f$ in (2.7). Clearly

$$
\left\|\Pi_{I} f\right\|_{s, r} \leq\|f\|_{s, r}
$$

and, for any $I, I^{\prime} \subset \mathbb{I}$, it results that

$$
\Pi_{I} \Pi_{I^{\prime}}=\Pi_{I \cap I^{\prime}}=\Pi_{I^{\prime}} \Pi_{I} .
$$

Property (2.14) is one of the main advantages of the majorant-norm with respect to the usual sup-norm

$$
|f|_{s, r}:=\sup _{v \in D(s, r)}|f(v)| .
$$

We now define useful projectors on the time Fourier indices. 
Definition 2.3. - Given $\varsigma=\left(\varsigma_{1}, \ldots, \varsigma_{n}\right) \in\{+,-\}^{n}$ we define

$$
f_{\varsigma}:=\Pi_{\varsigma} f:=\Pi_{\mathbb{Z}_{\varsigma}^{n} \times \mathbb{N}^{n} \times \mathbb{N}(\mathbb{Z} \backslash \mathcal{I}) \times \mathbb{N}(\mathbb{Z} \backslash \mathcal{I})} f=\sum_{k \in \mathbb{Z}_{\varsigma}^{n}, i, \alpha, \beta} f_{k, i, \alpha, \beta} e^{\mathrm{i} k \cdot x} y^{i} z^{\alpha} \bar{z}^{\beta}
$$

where

$$
\mathbb{Z}_{\varsigma}^{n}:=\left\{k \in \mathbb{Z}^{n} \quad \text { with } \quad\left\{\begin{array}{ll}
k_{h} \geq 0 & \text { if } \varsigma_{h}=+ \\
k_{h}<0 & \text { if } \quad \varsigma_{h}=-
\end{array} \quad \forall 1 \leq h \leq n\right\} .\right.
$$

Then any formal series $f$ can be decomposed as

$$
f=\sum_{\varsigma \in\{+,-\}^{n}} \Pi_{\varsigma} f
$$

and (2.14) implies $\left\|\Pi_{\varsigma} f\right\|_{s, r} \leq\|f\|_{s, r}$.

We now investigate the relations between formal power series with finite majorant norm and analytic functions. We recall that a function $f: D(s, r) \rightarrow \mathbb{C}$ is

- analytic, if $f \in C^{1}(D(s, r), \mathbb{C})$, namely the Fréchet differential $D(s, r) \ni v \mapsto d f(v) \in$ $\mathcal{L}(E, \mathbb{C})$ is continuous,

- weakly analytic, if $\forall v \in D(s, r), v^{\prime} \in E \backslash\{0\}$, there exists $\varepsilon>0$ such that the function

$$
\{\xi \in \mathbb{C},|\xi|<\varepsilon\} \mapsto f\left(v+\xi v^{\prime}\right) \in \mathbb{C}
$$

is analytic in the usual sense of one complex variable.

A well known result (see e.g., Theorem 1, page 133 of [29]) states that a function $f$ is

$$
\text { analytic } \Longleftrightarrow \text { weakly analytic and locally bounded . }
$$

Lemma 2.1. - Suppose that the formal power series (2.7) is absolutely convergent for all $v \in D(s, r)$. Then $f(v)$ and $M f(v)$, defined in (2.7) and (2.11), are well defined and weakly analytic in $D(s, r)$.

If, moreover, the sup-norm $|f|_{s, r}<\infty$, resp. $|M f|_{s, r}<\infty$, then $f$, resp. $M f$, is analytic in $D(s, r)$.

Proof. - Since the series (2.7) is absolutely convergent the functions $f, M f$, and, for all $\varsigma \in\{+,-\}^{n}, f_{\varsigma}:=\Pi_{\varsigma} f, M f_{\varsigma}$ (see (2.17)) are well defined (also the series in (2.17) is absolutely convergent).

We now prove that each $M f_{\varsigma}$ is weakly analytic, namely $\forall v \in D(s, r), v^{\prime} \in E \backslash\{0\}$,

$$
M f_{\varsigma}\left(v+\xi v^{\prime}\right)=\sum_{k \in \mathbb{Z}_{\varsigma}^{n}, i, \alpha, \beta}\left|f_{k, i, \alpha, \beta}\right| \mathfrak{m}_{k, i, \alpha, \beta}\left(v+\xi v^{\prime}\right)
$$

is analytic in $\{|\xi|<\varepsilon\}$, for $\varepsilon$ small enough (recall the notation (2.10)). Since each $\xi \mapsto \mathfrak{m}_{k, i, \alpha, \beta}\left(v+\xi v^{\prime}\right)$ is entire, the analyticity of $M f_{\varsigma}\left(v+\xi v^{\prime}\right)$ follows once we prove that the series (2.21) is totally convergent, namely

$$
\sum_{k \in \mathbb{Z}_{\varsigma}^{n}, i, \alpha, \beta}\left|f_{k, i, \alpha, \beta}\right| \sup _{|\xi|<\varepsilon}\left|\mathfrak{m}_{k, i, \alpha, \beta}\left(v+\xi v^{\prime}\right)\right|<+\infty .
$$

Let us prove (2.22). We claim that, for $\varepsilon$ small enough, there is $v^{\varsigma} \in D(s, r)$ such that

$$
\sup _{|\xi|<\varepsilon}\left|\mathfrak{m}_{k, i, \alpha, \beta}\left(v+\xi v^{\prime}\right)\right| \leq \mathfrak{m}_{k, i, \alpha, \beta}\left(v^{\varsigma}\right), \quad \forall k \in \mathbb{Z}_{\varsigma}^{n}, i, \alpha, \beta .
$$


Therefore (2.22) follows by

$$
\begin{aligned}
\sum_{k \in \mathbb{Z}_{\varsigma}^{n}, i, \alpha, \beta}\left|f_{k, i, \alpha, \beta}\right| \sup _{|\xi|<\varepsilon}\left|\mathfrak{m}_{k, i, \alpha, \beta}\left(v+\xi v^{\prime}\right)\right| & \leq \sum_{k \in \mathbb{Z}_{\varsigma}^{n}, i, \alpha, \beta}\left|f_{k, i, \alpha, \beta}\right| \mathfrak{m}_{k, i, \alpha, \beta}\left(v^{\varsigma}\right) \\
& =M f_{\varsigma}\left(v^{\varsigma}\right)<+\infty .
\end{aligned}
$$

Let us construct $v^{\varsigma} \in D(s, r)$ satisfying (2.23). Since $v=(x, y, z, \bar{z}) \in D(s, r)$ we have $x \in \mathbb{T}_{s}^{n}$ and, since $\mathbb{T}_{s}^{n}$ is open, there is $0<s^{\prime}<s$ such that $\left|\operatorname{Im}\left(x_{h}\right)\right|<s^{\prime}, \forall 1 \leq h \leq n$. Hence, for $\varepsilon$ small enough,

$$
\sup _{|\xi|<\varepsilon}\left|\operatorname{Im}\left(x+\xi x^{\prime}\right)_{h}\right| \leq s^{\prime}<s, \quad \forall 1 \leq h \leq n .
$$

The vector $v^{\varsigma}:=\left(x^{\varsigma}, y^{\varsigma}, z^{\varsigma}, \bar{z}^{\varsigma}\right)$ with components

$$
\begin{aligned}
& x_{h}^{\varsigma}:=-\mathrm{i} \varsigma_{h} s^{\prime}, \quad y_{h}^{\varsigma}:=\left|y_{h}\right|+\varepsilon\left|y_{h}^{\prime}\right|, \quad 1 \leq h \leq n, \\
& z_{h}^{\varsigma}:=\left|z_{h}\right|+\varepsilon\left|z_{h}^{\prime}\right|, \quad \bar{z}_{h}^{\varsigma}:=\left|\bar{z}_{h}\right|+\varepsilon\left|\bar{z}_{h}^{\prime}\right|, \quad h \in \mathbb{Z},
\end{aligned}
$$

belongs to $D(s, r)$ because $\left|\operatorname{Im} x_{h}^{\varsigma}\right|=s^{\prime}<s, \forall 1 \leq h \leq n$, and also $\left(y^{\varsigma}, z^{\varsigma}, \bar{z}^{\varsigma}\right) \in D(r)$ for $\varepsilon$ small enough, because $(y, z, \bar{z}) \in D(r)$ and $D(r)$ is open. Moreover, $\forall k \in \mathbb{Z}_{\varsigma}^{n}$, by (2.24), (2.18) and (2.25),

$$
\sup _{|\xi|<\varepsilon}\left|e^{\mathrm{i} k \cdot\left(x+\xi x^{\prime}\right)}\right| \leq e^{|k| s^{\prime}}=e^{\mathrm{i} k \cdot x^{\varsigma}} .
$$

By (2.10), (2.25), (2.26), we get (2.23). Hence each $M f_{\varsigma}$ is weakly analytic and, by the decomposition (2.19), also $f$ and $M f$ are weakly analytic. The final statement follows by (2.20).

COROLlary 2.1. - If $\|f\|_{s, r}<+\infty$ then $f$ and $M f$ are analytic and

$$
|f|_{s, r},|M f|_{s, r} \leq\|f\|_{s, r} .
$$

Proof. - For all $v=(x, y, z, \bar{z}) \in \mathbb{T}_{s}^{n} \times D(r)$, we have $\left|e^{\mathrm{i} k \cdot x}\right| \leq e^{|k| s}$ and

$$
|f(v)|,|M f(v)| \leq \sum_{k, i, \alpha, \beta}\left|f_{k, i, \alpha, \beta}\right| e^{|k| s}\left|y^{i}\right|\left|z^{\alpha}\right|\left|\bar{z}^{\beta}\right| \stackrel{(2.12)}{\leq}\|f\|_{s, r}<+\infty
$$

by assumption. Lemma 2.1 implies that $f, M f$ are analytic.

Now, we associate to any analytic function $f: D(s, r) \rightarrow \mathbb{C}$ the formal Taylor-Fourier power series

$$
\mathbf{f}(v):=\sum_{(k, i, \alpha, \beta) \in \mathbb{I}} f_{k, i, \alpha, \beta} e^{\mathrm{i} k \cdot x} y^{i} z^{\alpha} \bar{z}^{\beta}
$$

(as (2.7)) with Taylor-Fourier coefficients

$$
f_{k, i, \alpha, \beta}:=\frac{1}{(2 \pi)^{n}} \int_{\mathbb{T}^{n}} e^{-\mathrm{i} k \cdot x} \frac{1}{i ! \alpha ! \beta !}\left(\partial_{y}^{i} \partial_{z}^{\alpha} \partial_{\bar{z}}^{\beta} f\right)(x, 0,0,0) d x
$$

where $\partial_{y}^{i} \partial_{z}^{\alpha} \partial_{\bar{z}}^{\beta} f$ are the partial derivatives ${ }^{(1)}$.

(1) For a multi-index $\alpha=\sum_{1 \leq j \leq k} e_{i_{j}},|\alpha|=k$, the partial derivative is

$$
\partial_{z}^{\alpha} f(x, y, z, \bar{z}):=\frac{\partial^{k}}{\partial \tau_{1} \ldots \partial \tau_{k}}{ }_{\mid \tau=0} f\left(x, y, z+\tau_{1} e_{i_{1}}+\cdots+\tau_{k} e_{i_{k}}, \bar{z}\right) .
$$


What is the relation between $f$ and its formal Taylor-Fourier series $\mathbf{f}$ ?

Lemma 2.2. - Let $f: D(s, r) \rightarrow \mathbb{C}$ be analytic. If its associated Taylor-Fourier power series (2.28)-(2.29) is absolutely convergent in $D(s, r)$, and the sup-norm

$$
\left|\sum_{k, i, \alpha, \beta} f_{k, i, \alpha, \beta} e^{\mathrm{i} k \cdot x} y^{i} z^{\alpha} \bar{z}^{\beta}\right|_{s, r}<\infty
$$

then $f=\mathbf{f}, \forall v \in D(s, r)$.

Proof. - Since the Taylor-Fourier series (2.28)-(2.29) is absolutely convergent and (2.31) holds, by Lemma 2.1 the function $\mathrm{f}: D(s, r) \rightarrow \mathbb{C}$ is analytic. The functions $f=\mathrm{f}$ are equal if the Taylor-Fourier coefficients

$$
f_{k, i, \alpha, \beta}=\mathrm{f}_{k, i, \alpha, \beta}, \quad \forall k, i, \alpha, \beta,
$$

where the coefficients $f_{k, i, \alpha, \beta}$ are defined from $f$ as in (2.29). Let us prove (2.32). Indeed, for example,

$$
\begin{aligned}
\mathbf{f}_{0,0, e_{h}, 0} & =\frac{1}{(2 \pi)^{n}} \int_{\mathbb{T}^{n}} \frac{d}{d \xi} \sum_{\mid \xi=0} \sum_{k \in \mathbb{Z}^{n}, m \in \mathbb{N}} f_{k, 0, m e_{h}, 0} e^{\mathrm{i} k \cdot x} \xi^{m} \\
& =\sum_{k \in \mathbb{Z}^{n}, m \in \mathbb{N}} \frac{1}{(2 \pi)^{n}} \int_{\mathbb{T}^{n}} \frac{d}{d \xi} f_{k, 0, m e_{h}, 0} e^{\mathrm{i} k \cdot x} \xi^{m}=f_{0,0, e_{h}, 0},
\end{aligned}
$$

using that the above series totally converge for $r^{\prime}<r$, namely

$$
\begin{aligned}
\sum_{k \in \mathbb{Z}^{n}, m \in \mathbb{N}} \sup _{x \in \mathbb{R},|\xi| \leq r^{\prime}}\left|f_{k, 0, m e_{h}, 0} e^{\mathrm{i} k \cdot x} \xi^{m}\right| & \leq \sum_{k \in \mathbb{Z}^{n}, m \in \mathbb{N}}\left|f_{k, 0, m e_{h}, 0}\right|\left(r^{\prime}\right)^{m} \\
& \leq \sum_{k, i, \alpha, \beta}\left|f_{k, i, \alpha, \beta} \mathfrak{m}_{k, i, \alpha, \beta}\left(0,0, r^{\prime} e_{h}, 0\right)\right|<\infty
\end{aligned}
$$

recall (2.10). For the others $k, i, \alpha, \beta$ in (2.32) are analogous.

The above arguments also show the unicity of the Taylor-Fourier expansion.

Lemma 2.3. - If an analytic function $f: D(s, r) \rightarrow \mathbb{C}$ equals an absolutely convergent formal series, i.e., $f(v)=\sum_{k, i, \alpha, \beta} \tilde{f}_{k, i, \alpha, \beta} e^{\mathrm{i} k \cdot x} y^{i} z^{\alpha} \bar{z}^{\beta}$, then its Taylor-Fourier coefficients (2.29) are $f_{k, i, \alpha, \beta}=\tilde{f}_{k, i, \alpha, \beta}$.

The majorant norm of $f$ is equivalent to the sup-norm of its majorant $M f$.

LEMMA 2.4. -

$$
|M f|_{s, r} \leq\|f\|_{s, r} \leq 2^{n}|M f|_{s, r} .
$$

Proof. - The first inequality in (2.34) is (2.27). The second one follows by

$$
\left\|\Pi_{\varsigma} f\right\|_{s, r} \leq|M f|_{s, r}, \quad \forall \varsigma \in\{+,-\}^{n},
$$

where $\Pi_{\varsigma} f$ is defined in (2.17). Let us prove (2.35). Let

$$
D^{+}(r):=\left\{(y, z, \bar{z}) \in D(r): y_{h} \geq 0, \forall 1 \leq h \leq n, z_{l}, \bar{z}_{l} \geq 0, \forall l \in \mathbb{Z} \backslash \mathcal{I}\right\} .
$$


For any $0 \leq \sigma<s$, we have

$$
\begin{aligned}
|M f|_{s, r} & =\sup _{(x, y, z, \bar{z}) \in D(s, r)}\left|\sum_{k, i, \alpha, \beta}\right| f_{k, i, \alpha, \beta}\left|e^{\mathrm{i} k \cdot x} y^{i} z^{\alpha} \bar{z}^{\beta}\right| \\
& \geq \sup _{x_{1}=-\mathrm{i} \varsigma_{1} \sigma, \ldots, x_{n}=-\mathrm{i} \varsigma_{n} \sigma,(y, z, \bar{z}) \in D^{+}(r)}\left|\sum_{k, i, \alpha, \beta}\right| f_{k, i, \alpha, \beta}\left|e^{\mathrm{i} k \cdot x} y^{i} z^{\alpha} \bar{z}^{\beta}\right| \\
& \stackrel{(2.18)}{\geq} \sup _{(y, z, \bar{z}) \in D^{+}(r)} \sum_{k \in \mathbb{Z}_{\varsigma}^{n}, i, \alpha, \beta}\left|f_{k, i, \alpha, \beta}\right| e^{|k| \sigma}\left|y^{i}\right|\left|z^{\alpha}\right|\left|\bar{z}^{\beta}\right| \\
& =\sup _{(y, z, \bar{z}) \in D(r)} \sum_{k \in \mathbb{Z}_{\varsigma}^{n}, i, \alpha, \beta}\left|f_{k, i, \alpha, \beta}\right| e^{|k| \sigma}\left|y^{i}\right|\left|z^{\alpha}\right|\left|\bar{z}^{\beta}\right|=\left\|\Pi_{\varsigma} f\right\|_{\sigma, r} .
\end{aligned}
$$

Then (2.35) follows since for every function $g$ we have $\sup _{0 \leq \sigma<s}\|g\|_{\sigma, r}=\|g\|_{s, r}$.

Definition 2.4 (Order relation: scalar functions). - Given formal power series

$$
f=\sum_{k, i, \alpha, \beta} f_{k, i, \alpha, \beta} e^{\mathrm{i} k \cdot x} y^{i} z^{\alpha} \bar{z}^{\beta}, g=\sum_{k, i, \alpha, \beta} g_{k, i, \alpha, \beta} e^{\mathrm{i} k \cdot x} y^{i} z^{\alpha} \bar{z}^{\beta},
$$

with $g_{k, i, \alpha, \beta} \in \mathbb{R}^{+}$, we say that

$$
f \prec g \quad \text { if } \quad\left|f_{k, i, \alpha, \beta}\right| \leq g_{k, i, \alpha, \beta}, \forall k, i, \alpha, \beta .
$$

Note that, by the Definition (2.11) of majorant series,

$$
f \prec g \quad \Longleftrightarrow \quad f \prec M f \prec g .
$$

Moreover, if $\|g\|_{s, r}<+\infty$, then $f \prec g \Longrightarrow\|f\|_{s, r} \leq\|g\|_{s, r}$.

For any $\varsigma \in\{+,-\}^{n}$ define $q_{\varsigma}:=\left(q_{\varsigma}^{(j)}\right)_{j \in \mathcal{J}}$ as

$$
q_{\varsigma}^{(j)}:= \begin{cases}-\varsigma_{h} \mathrm{i} & \text { if } j=(1, h), 1 \leq h \leq n, \\ 1 & \text { otherwise }\end{cases}
$$

Lemma 2.5. - Assume $\|f\|_{s, r},\|g\|_{s, r}<+\infty$. Then

$$
f+g \prec M f+M g, \quad f \cdot g \prec M f \cdot M g
$$

and

$$
M\left(\partial_{j}\left(\Pi_{\varsigma} f\right)\right)=q_{\varsigma}^{(j)} \partial_{j}\left(M\left(\Pi_{\varsigma} f\right)\right), \quad j \in \mathcal{J},
$$

where $\partial_{j}$ is short for $\partial_{v^{(j)}}$ and $q_{\varsigma}^{(j)}$ are defined in (2.38).

Proof. - Since the series which define $f$ and $g$ are absolutely convergent, the bounds (2.39) follow by summing and multiplying the series term by term. Next (2.40) follows by differentiating the series term by term.

An immediate consequence of (2.39) is

$$
\|f+g\|_{s, r} \leq\|f\|_{s, r}+\|g\|_{s, r}, \quad\|f g\|_{s, r} \leq\|f\|_{s, r}\|g\|_{s, r} .
$$

The next lemma extends property (2.39) for infinite series.

Lemma 2.6. - Assume that $f^{(j)}, g^{(j)}$ are formal power series satisfying

1. $f^{(j)} \prec g^{(j)}, \forall j \in \mathcal{J}$,

2. $\left\|g^{(j)}\right\|_{s, r}<\infty, \forall j \in \mathcal{J}$,

$4^{\text {e }}$ SÉRIE - TOME 46 - 2013 - No 2 
3. $\sum_{j \in \mathcal{J}}\left|g^{(j)}(v)\right|<\infty, \forall v \in D(s, r)$,

4. $g(v):=\sum_{j \in \mathcal{J}} g^{(j)}(v)$ is bounded in $D(s, r)$, namely $|g|_{s, r}<\infty$.

Then the function $g: D(s, r) \rightarrow \mathbb{C}$ is analytic, its Taylor-Fourier coefficients (defined as in (2.29)) are

$$
g_{k, i, \alpha, \beta}=\sum_{j \in \mathcal{J}} g_{k, i, \alpha, \beta}^{(j)} \geq 0, \quad \forall(k, i, \alpha, \beta) \in \mathbb{I},
$$

and $\|g\|_{s, r}<\infty$. Moreover

1. $\sum_{j \in \mathcal{J}}\left|f^{(j)}(v)\right|<\infty, \forall v \in D(s, r)$,

2. $f(v):=\sum_{j \in \mathcal{J}} f^{(j)}(v)$ is analytic in $D(s, r)$,

3. $f \prec g$ and $\|f\|_{s, r} \leq\|g\|_{s, r}<\infty$.

Proof. - For each monomial $\mathfrak{m}_{k, i, \alpha, \beta}(v)$ (see (2.10)) and $v=(x, y, z, \bar{z}) \in D(s, r)$, we have

$$
\left|\mathfrak{m}_{k, i, \alpha, \beta}(v)\right|=\mathfrak{m}_{k, i, \alpha, \beta}\left(v_{+}\right),
$$

where $v_{+}:=(\mathrm{i} \operatorname{Im} x,|y|,|z|,|\bar{z}|) \in D(s, r)$ with $|y|:=\left(\left|y_{1}\right|, \ldots,\left|y_{n}\right|\right)$ and $|z|,|\bar{z}|$ are similarly defined.

Since $\left\|g^{(j)}\right\|_{s, r}<\infty$ (and $\left.f^{(j)} \prec g^{(j)}\right)$ the series

$$
g^{(j)}(v):=\sum_{k, i, \alpha, \beta} g_{k, i, \alpha, \beta}^{(j)} \mathfrak{m}_{k, i, \alpha, \beta}(v), \quad g_{k, i, \alpha, \beta}^{(j)} \geq 0
$$

is absolutely convergent. For all $v \in D(s, r)$ we prove that

$$
\begin{aligned}
\sum_{j \in \mathcal{J}} \sum_{k, i, \alpha, \beta}\left|g_{k, i, \alpha, \beta}^{(j)} \mathfrak{m}_{k, i, \alpha, \beta}(v)\right| & \stackrel{(2.44),(2.43)}{=} \sum_{j \in \mathcal{J}} \sum_{k, i, \alpha, \beta} g_{k, i, \alpha, \beta}^{(j)} \mathfrak{m}_{k, i, \alpha, \beta}\left(v_{+}\right) \\
\stackrel{(2.44)}{=} & \sum_{j \in \mathcal{J}} g^{(j)}\left(v_{+}\right)=g\left(v_{+}\right)<\infty
\end{aligned}
$$

by assumption 3. Therefore, by Fubini's theorem, we exchange the order of the series

$$
g(v)=\sum_{j \in \mathcal{J}} \sum_{k, i, \alpha, \beta} g_{k, i, \alpha, \beta}^{(j)} \mathfrak{m}_{k, i, \alpha, \beta}(v)=\sum_{k, i, \alpha, \beta}\left(\sum_{j \in \mathcal{J}} g_{k, i, \alpha, \beta}^{(j)}\right) \mathfrak{m}_{k, i, \alpha, \beta}(v)
$$

proving that $g$ is equal to an absolutely convergent series. Lemma 2.1 and the assumption $|g|_{s, r}<\infty$ imply that $g$ is analytic in $D(s, r)$. Moreover (2.46) and Lemma 2.3 imply (2.42). The $g_{k, i, \alpha, \beta} \geq 0$ because $g_{k, i, \alpha, \beta}^{(j)} \geq 0$, see (2.44). Therefore $M g=g$, and, by (2.34) and the assumption $|g|_{s, r}<\infty$, we deduce $\|g\|_{s, r}<\infty$.

Concerning $f$ we have

$$
\sum_{j \in \mathcal{J}}\left|f^{(j)}(v)\right| \leq \sum_{j \in \mathcal{J}} \sum_{k, i, \alpha, \beta}\left|f_{k, i, \alpha, \beta}^{(j)} \mathfrak{m}_{k, i, \alpha, \beta}(v)\right| \leq \sum_{j \in \mathcal{J}} \sum_{k, i, \alpha, \beta} g_{k, i, \alpha, \beta}^{(j)}\left|\mathfrak{m}_{k, i, \alpha, \beta}(v)\right| \stackrel{(2.45)}{<} \infty
$$

and, arguing as for $g$, its Taylor-Fourier coefficients are

$$
f_{k, i, \alpha, \beta}=\sum_{j \in \mathcal{J}} f_{k, i, \alpha, \beta}^{(j)}, \forall(k, i, \alpha, \beta) \in \mathbb{I} .
$$

Then

$$
\left|f_{k, i, \alpha, \beta}\right| \leq \sum_{j \in \mathcal{J}}\left|f_{k, i, \alpha, \beta}^{(j)}\right| \leq \sum_{j \in \mathcal{J}} g_{k, i, \alpha, \beta}^{(j)} \stackrel{(2.42)}{=} g_{k, i, \alpha, \beta}
$$


Hence $f \prec g$ and $\|f\|_{s, r} \leq\|g\|_{s, r}<\infty$. Finally $f$ is analytic by Lemma 2.1.

Lemma 2.7. - Let $\|f\|_{s, r}<\infty$. Then, $\forall 0<s^{\prime}<s, 0<r^{\prime}<r$, we have $\left\|\partial_{j} f\right\|_{s^{\prime}, r^{\prime}}<\infty$.

Proof. - It is enough to prove the lemma for each $f_{\varsigma}=\Pi_{\varsigma} f$ defined in (2.17). By $\|f\|_{s, r}<\infty$ and Corollary 2.1 the functions $f_{\varsigma}, M f_{\varsigma}$ are analytic and

$$
\left\|\partial_{j} f_{\varsigma}\right\|_{s^{\prime}, r^{\prime}} \stackrel{(2.34)}{\leq} 2^{n}\left|M\left(\partial_{j} f_{\varsigma}\right)\right|_{s^{\prime}, r^{\prime}} \stackrel{(2.40)}{=} 2^{n}\left|\partial_{j}\left(M f_{\varsigma}\right)\right|_{s^{\prime}, r^{\prime}} \leq c\left|M f_{\varsigma}\right|_{s, r} \stackrel{(2.34)}{\leq} c\left\|f_{\varsigma}\right\|_{s, r}
$$

for a suitable $c:=c\left(n, s, s^{\prime}, r, r^{\prime}\right)$, having used the Cauchy estimate (in one variable).

We conclude this subsection with a simple result on representation of differentials.

Lemma 2.8. - Let $f: D(s, r) \rightarrow \mathbb{C}$ be Fréchet differentiable at $v_{0}$. Then

$$
d f\left(v_{0}\right)[v]=\sum_{j \in \mathcal{J}} \partial_{j} f\left(v_{0}\right) v^{(j)}, \quad \forall v=\sum_{j \in \mathcal{J}} v^{(j)} e_{j} \in E
$$

and

$$
\sum_{j \in \mathcal{J}}\left|\partial_{j} f\left(v_{0}\right) v^{(j)}\right| \leq\left\|d f\left(v_{0}\right)\right\|_{\mathcal{L}(E, \mathbb{C})}\|v\|_{E}
$$

Proof. - (2.47) follows by the continuity of the differential $d f\left(v_{0}\right) \in \mathcal{L}(E, \mathbb{C})$. Next, consider a vector $\tilde{v}=\left(\tilde{v}^{(j)}\right)_{j \in \mathcal{J}} \in E$ such that $\left|\tilde{v}_{j}\right|=\left|v_{j}\right|$ and

$$
\tilde{v}^{(j)}\left(\partial_{j} f\right)\left(v_{0}\right)=\left|\left(\partial_{j} f\right)\left(v_{0}\right) v^{(j)}\right|, \quad \forall j \in \mathcal{J} .
$$

Hence $d f\left(v_{0}\right)[\tilde{v}]=\sum_{j \in \mathcal{J}} \tilde{v}^{(j)}\left(\partial_{j} f\right)\left(v_{0}\right)=\sum_{j \in \mathcal{J}}\left|\left(\partial_{j} f\right)\left(v_{0}\right) v^{(j)}\right|$ which gives (2.48) because $\|\tilde{v}\|_{E}=\|v\|_{E}$.

2.1.2. Vector fields. - We now consider a formal vector field

$$
X(v):=\left(X^{(j)}(v)\right)_{j \in \mathcal{J}}
$$

where each component $X^{(j)}$ is a formal power series

$$
X^{(j)}(v)=X^{(j)}(x, y, z, \bar{z})=\sum_{k, i, \alpha, \beta} X_{k, i, \alpha, \beta}^{(j)} e^{\mathrm{i} k \cdot x} y^{i} z^{\alpha} \bar{z}^{\beta}
$$

as in (2.7). We define its "majorant" vector field componentwise, namely

$$
M X(v):=\left((M X)^{(j)}(v)\right)_{j \in \mathcal{J}}:=\left(M X^{(j)}(v)\right)_{j \in \mathcal{J}} .
$$

We consider vector fields $X: D(s, r) \subset E \rightarrow E$, see (2.1).

Definition 2.5. - The vector field $X$ is absolutely convergent at $v$ if every component $X^{(j)}(v), j \in \mathcal{J}$, is absolutely convergent (see Definition 2.1) and

$$
\left\|\left(X^{(j)}(v)\right)_{j \in \mathcal{J}}\right\|_{E}<+\infty .
$$

The properties of the space $E$ in (2.1) (as target space), that we will use are:

1. $E$ is a separable Hilbert space times a finite dimensional space,

2. the "monotonicity property" of the norm

$$
v_{0}, v_{1} \in E \quad \text { with }\left|v_{0}^{(j)}\right| \leq\left|v_{1}^{(j)}\right|, \forall j \in \mathcal{J} \quad \Longrightarrow \quad\left\|v_{0}\right\|_{E} \leq\left\|v_{1}\right\|_{E} .
$$


For $X: D(s, r) \rightarrow E$ we define the sup-norm

$$
|X|_{s, r}:=\sup _{v \in D(s, r)}\|X(v)\|_{E, s, r} .
$$

Definition 2.6 (Majorant-norm: vector field). - The majorant norm of a formal vector field $X$ as in (2.49) is

$$
\begin{aligned}
\|X\|_{s, r} & :=\sup _{(y, z, \bar{z}) \in D(r)}\left\|\left(\sum_{k, i, \alpha, \beta}\left|X_{k, i, \alpha, \beta}^{(j)}\right| e^{|k| s}\left|y^{i}\right|\left|z^{\alpha}\right|\left|\bar{z}^{\beta}\right|\right)_{j \in \mathcal{J}}\right\|_{E, s, r} \\
& =\sup _{(y, z, \bar{z}) \in D(r)}\left\|\sum _ { k , i , \alpha , \beta } | X _ { k , i , \alpha , \beta } | e ^ { | k | s } \left|y^{i}\left\|z^{\alpha}|| \bar{z}^{\beta} \mid\right\|_{E, s, r}\right.\right.
\end{aligned}
$$

where

$$
X_{k, i, \alpha, \beta}:=\left(X_{k, i, \alpha, \beta}^{(j)}\right)_{j \in \mathcal{J}} \quad \text { and } \quad\left|X_{k, i, \alpha, \beta}\right|:=\left(\left|X_{k, i, \alpha, \beta}^{(j)}\right|\right)_{j \in \mathcal{J}} .
$$

REMARK 2.3. - The stronger norm (see [27])

$$
\mid X\left\|_{s, r}:=\right\|\left(\sup _{(y, z, \bar{z}) \in D(r)} \sum_{k, i, \alpha, \beta}\left|X_{k, i, \alpha, \beta}^{(j)}\right| e^{|k| s}\left|y^{i} \| z^{\alpha}\right|\left|\bar{z}^{\beta}\right|\right)_{j \in \mathcal{J}} \|_{E, s, r}
$$

is not suited for infinite dimensional systems: for $X=I d$ we have $\|\left. X\right|_{s, r}=+\infty$.

By (2.54) and (2.51) we get $\|X\|_{s, r}=\|M X\|_{s, r}$. For a subset of indices $I \subset \mathbb{I}$ we define the projection

$$
\left(\Pi_{I} X\right)(x, y, z, \bar{z}):=\sum_{(k, i, \alpha, \beta) \in I} X_{k, i, \alpha, \beta} e^{\mathrm{i} k \cdot x} y^{i} z^{\alpha} \bar{z}^{\beta}
$$

Lemma 2.9 (Projection). $-\forall I \subset \mathbb{I}$,

$$
\left\|\Pi_{I} X\right\|_{s, r} \leq\|X\|_{s, r} .
$$

Proof. - See (2.54).

REMARK 2.4. - The estimate (2.55) may fail for the sup-norm ||$_{s, r}$ and suitable I.

Let us define the "ultraviolet" resp. infrared projections

$$
\left(\Pi_{|k| \geq K} X\right)(x, y, z, \bar{z}):=\sum_{|k| \geq K, i, \alpha, \beta} X_{k, i, \alpha, \beta} e^{\mathrm{i} k \cdot x} y^{i} z^{\alpha} \bar{z}^{\beta}, \quad \Pi_{|k|<K}:=I d-\Pi_{|k| \geq K}
$$

Lemma 2.10 (Smoothing). $-\forall 0<s^{\prime}<s$,

$$
\left\|\Pi_{|k| \geq K} X\right\|_{s^{\prime}, r} \leq \frac{s}{s^{\prime}} e^{-K\left(s-s^{\prime}\right)}\|X\|_{s, r} .
$$

Proof. - Recall (2.54) and use $e^{|k| s^{\prime}} \leq e^{|k| s} e^{-K\left(s-s^{\prime}\right)}, \forall|k| \geq K$. 
We decompose each formal vector field

$$
X=\sum_{\varsigma \in\{+,-\}^{n}} \Pi_{\varsigma} X
$$

applying (2.19) componentwise

$$
X_{\varsigma}:=\Pi_{\varsigma} X:=\left(\Pi_{\varsigma} X^{(j)}\right)_{j \in \mathcal{J}}
$$

recall (2.17). Clearly (2.55) implies

$$
\left\|X_{\varsigma}\right\|_{s, r} \leq\|X\|_{s, r} .
$$

In the next lemma we prove that, if $X$ has finite majorant norm, then it is analytic.

Lemma 2.11. - Assume

$$
\|X\|_{s, r}<+\infty .
$$

Then the series in (2.49)-(2.50), resp. (2.51), absolutely converge to the analytic vector field $X(v)$, resp. $M X(v)$, for every $v \in D(s, r)$. Moreover the sup-norm defined in (2.53) satisfies

$$
|X|_{s, r},|M X|_{s, r} \leq\|X\|_{s, r} .
$$

Proof. - By (2.61) and Definition 2.6, for each $j \in \mathcal{J}$, we have

$$
\sup _{(y, z, \bar{z}) \in D(r)} \sum_{k, i, \alpha, \beta}\left|X_{k, i, \alpha, \beta}^{(j)}\right| e^{|k| s}\left|y^{i}\right|\left|z^{\alpha}\right|\left|\bar{z}^{\beta}\right|<+\infty
$$

and Lemma 2.1 (and Corollary 2.1) implies that each coordinate function $X^{(j)}$, $(M X)^{(j)}: D(s, r) \rightarrow \mathbb{C}$ is analytic. Moreover (2.62) follows applying (2.27) componentwise. By (2.61) the maps

$$
X, M X: D(s, r) \rightarrow E
$$

are bounded. Since $E$ is a separable Hilbert space (times a finite dimensional space), Theorem 3-Appendix A in [29], implies that $X, M X: D(s, r) \rightarrow E$ are analytic.

Viceversa, we associate to an analytic vector field $X: D(s, r) \rightarrow E$ a formal TaylorFourier vector field (2.49)-(2.50) developing each component $X^{(j)}$ as in (2.28)-(2.29).

Definition 2.7 (Order relation: vector fields). - Given formal vector fields $X, Y$, we say that

$$
X \prec Y
$$

if each coordinate $X^{(j)} \prec Y^{(j)}, j \in \mathcal{J}$, according to Definition 2.4.

If $\|Y\|_{s, r}<+\infty$ and

$$
X \prec Y \quad \Longrightarrow \quad\|X\|_{s, r} \leq\|Y\|_{s, r} .
$$

Applying Lemma 2.5 componentwise we get

Lemma 2.12. - If $\|X\|_{s, r},\|Y\|_{s, r}<\infty$ then $X+Y \prec M X+M Y$ and $\|X+Y\|_{s, r} \leq$ $\|X\|_{s, r}+\|Y\|_{s, r}$.

LEMMA 2.13. -

$$
|M X|_{s, r} \leq\|X\|_{s, r} \leq 2^{n}|M X|_{s, r} .
$$

$4^{\mathrm{e}}$ SÉRIE - TOME $46-2013$ - No 2 

(2.52).

Proof. - As for Lemma 2.4 with $f \rightsquigarrow X,\left|\sum_{k, i, \alpha, \beta}\right| \rightsquigarrow\left\|\sum_{k, i, \alpha, \beta}\right\|_{E}$ and using

We define the space of analytic vector fields

$$
\mathcal{V}_{s, r}:=\mathcal{V}_{s, r, E}:=\left\{X: D(s, r) \rightarrow E \text { with norm }\|X\|_{s, r}<+\infty\right\} .
$$

By Lemma 2.11 if $X \in \mathcal{V}_{s, r}$ then $X$ is analytic, namely the Fréchet differential $D(s, r) \ni v \mapsto d X(v) \in \mathcal{L}(E, E)$ is continuous. The next lemma bounds its operator norm from $(E, s, r):=\left(E,\|\|_{E, s, r}\right)$ to $\left(E, s^{\prime}, r^{\prime}\right)$, see (2.2).

Lemma 2.14 (Cauchy estimate). - Let $X \in \mathcal{V}_{s, r}$. Then, for $s / 2 \leq s^{\prime}<s, r / 2 \leq r^{\prime}<r$,

$$
\sup _{v \in D\left(s^{\prime}, r^{\prime}\right)}\|d X(v)\|_{\mathcal{L}\left((E, s, r),\left(E, s^{\prime}, r^{\prime}\right)\right)} \leq 4 \delta^{-1}|X|_{s, r}
$$

where the sup-norm $|X|_{s, r}$ is defined in (2.53) and

$$
\delta:=\min \left\{1-\frac{s^{\prime}}{s}, 1-\frac{r^{\prime}}{r}\right\} .
$$

Proof. - In the appendix.

The commutator of two vector fields $X, Y: D(s, r) \rightarrow E$ is

$$
[X, Y](v):=d X(v)[Y(v)]-d Y(v)[X(v)], \quad \forall v \in D(s, r) .
$$

The next lemma is the fundamental result of this section.

Lemma 2.15 (Commutator). - Let $X, Y \in \mathcal{V}_{s, r}$. Then, for $r / 2 \leq r^{\prime}<r, s / 2 \leq s^{\prime}<s$,

$$
\|[X, Y]\|_{s^{\prime}, r^{\prime}} \leq 2^{2 n+3} \delta^{-1}\|X\|_{s, r}\|Y\|_{s, r}
$$

where $\delta$ is defined in (2.66).

Proof. - The lemma follows by

$$
\|d X[Y]\|_{s^{\prime}, r^{\prime}} \leq 4^{n+2} \delta^{-1}\|X\|_{s, r}\|Y\|_{s, r},
$$

the analogous estimate for $d Y[X]$ and (2.67).

We claim that, for each $\varsigma \in\{+,-\}^{n}$, the vector field $X_{\varsigma}$ defined in (2.59) satisfies

$$
\left\|d X_{\varsigma}[Y]\right\|_{s^{\prime}, r^{\prime}} \leq 2^{n+2} \delta^{-1}\left\|X_{\varsigma}\right\|_{s, r}\|Y\|_{s, r}
$$

which implies (2.69) because

$$
\begin{aligned}
\|d X[Y]\|_{s^{\prime}, r^{\prime}} & \stackrel{(2.58)}{\leq} \sum_{\varsigma \in\{+,-\}^{n}}\left\|d X_{\varsigma}[Y]\right\|_{s^{\prime}, r^{\prime}} \stackrel{(2.70)}{\leq} \sum_{\varsigma \in\{+,-\}^{n}} 2^{n+2} \delta^{-1}\left\|X_{\varsigma}\right\|_{s, r}\|Y\|_{s, r} \\
& \stackrel{(2.60)}{\leq} \sum_{\varsigma \in\{+,-\}^{n}} 2^{n+2} \delta^{-1}\|X\|_{s, r}\|Y\|_{s, r} \leq 4^{n+2} \delta^{-1}\|X\|_{s, r}\|Y\|_{s, r} .
\end{aligned}
$$

Let us prove (2.70). First note that, since $\left\|X_{\varsigma}\right\|_{s, r} \stackrel{(2.60)}{\leq}\|X\|_{s, r}<+\infty$ and $\|Y\|_{s, r}<+\infty$ by assumption, Lemma 2.11 implies that the vector fields

$$
X_{\varsigma}, M X_{\varsigma}, Y, M Y: D(s, r) \rightarrow E, \quad \forall \varsigma \in\{+,-\}^{n},
$$

are analytic, as well as each component $X_{\varsigma}^{(i)}, M X_{\varsigma}^{(i)}, Y^{(i)}, M Y^{(i)}: D(s, r) \rightarrow \mathbb{C}, i \in \mathcal{J}$. 
The key for proving the lemma is the following chain of inequalities:

$$
\begin{aligned}
d X_{\varsigma}[Y]^{(i)} \prec M\left(d X_{\varsigma}[Y]\right)^{(i)} \stackrel{(2.47)}{=} M\left(\sum_{j \in \mathcal{J}}\left(\partial_{j} X_{\varsigma}^{(i)}\right) Y^{(j)}\right) \\
\stackrel{\text { Lemma }^{2}}{2.6} \sum_{j \in \mathcal{J}} M\left(\partial_{j} X_{\varsigma}^{(i)}\right) M Y^{(j)} \\
\stackrel{(2.40)}{=} \sum_{j \in \mathcal{J}} q_{\varsigma}^{(j)} \partial_{j}\left(M X_{\varsigma}^{(i)}\right) M Y^{(j) \stackrel{(2.47)}{=}} d\left(M X_{\varsigma}^{(i)}\right)\left[\tilde{Y}_{q}\right]
\end{aligned}
$$

where

$$
\tilde{Y}_{q}:=\left(\tilde{Y}_{q}^{(j)}\right)_{j \in \mathcal{J}}:=\left(q_{\varsigma}^{(j)} M Y^{(j)}\right)_{j \in \mathcal{J}} \in E
$$

Actually, since $\left|q_{\varsigma}^{(j)}\right|=1$ (see (2.38)), then

$$
\left\|\tilde{Y}_{q}(v)\right\|_{E}=\|M Y(v)\|_{E} \stackrel{(2.71)}{<}+\infty, \quad \forall v \in D(s, r) .
$$

In (2.72) above we applied Lemma 2.6 with

$$
s \rightsquigarrow s^{\prime}, r \rightsquigarrow r^{\prime}, f^{(j)} \rightsquigarrow\left(\partial_{j} X_{\varsigma}^{(i)}\right) Y^{(j)}, g^{(j)} \rightsquigarrow M\left(\partial_{j} X_{\varsigma}^{(i)}\right) M Y^{(j)} .
$$

Let us verify that the hypotheses of Lemma 2.6 hold:

1. $f^{(j)} \prec g^{(j)}$ follows by (2.39) and since $\left\|f^{(j)}\right\|_{s^{\prime}, r^{\prime}},\left\|g^{(j)}\right\|_{s^{\prime}, r^{\prime}}<+\infty$ because $\left\|X_{\varsigma}^{(i)}\right\|_{s, r} \leq\|X\|_{s, r}<+\infty,\left\|Y^{(j)}\right\|_{s, r} \leq\|Y\|_{s, r}<+\infty$, and Lemma 2.7 .

2. $\left\|g^{(j)}\right\|_{s^{\prime}, r^{\prime}}<\infty$ is proved above.

3. We have $\sum_{j \in \mathcal{J}}\left|g^{(j)}(v)\right|<\infty$, for all $v \in D\left(s^{\prime}, r^{\prime}\right)$, because

$$
\begin{aligned}
\sum_{j \in \mathcal{J}}\left|g^{(j)}(v)\right| & \stackrel{(2.75)}{=} \sum_{j \in \mathcal{J}}\left|M\left(\partial_{j} X_{\varsigma}^{(i)}\right)(v) M Y^{(j)}(v)\right| \stackrel{(2.40)}{=} \sum_{j \in \mathcal{J}}\left|q_{\varsigma}^{(j)} \partial_{j}\left(M X_{\varsigma}^{(i)}\right)(v) M Y^{(j)}(v)\right| \\
\stackrel{(2.38)}{=} & \sum_{j \in \mathcal{J}}\left|\partial_{j}\left(M X_{\varsigma}^{(i)}\right)(v) M Y^{(j)}(v)\right| \stackrel{(2.48)}{\leq}\left\|d M X_{\varsigma}^{(i)}(v)\right\|_{\mathcal{L}(E, \mathbb{C})}\|M Y(v)\|_{E}<+\infty
\end{aligned}
$$

by (2.71), (2.74). Actually we also proved that $g^{(j)}=q_{\varsigma}^{(j)} \partial_{j}\left(M X_{\varsigma}^{(i)}\right) M Y^{(j)}$.

4. The function

$$
g(v):=\sum_{j \in \mathcal{J}} g^{(j)}(v)=\sum_{j \in \mathcal{J}} q_{\varsigma}^{(j)} \partial_{j}\left(M X_{\varsigma}^{(i)}\right) M Y^{(j)} \stackrel{(2.47)}{=} d\left(M X_{\varsigma}^{(i)}\right)\left[\tilde{Y}_{q}\right]
$$

since $M X_{\varsigma}^{(i)}$ is differentiable (see (2.71)) and $\tilde{Y}_{q} \in E$ (see (2.74)).

Moreover the bound $|g|_{s^{\prime}, r^{\prime}}<\infty$ follows by

$$
|g|_{s^{\prime}, r^{\prime}}=\left|d\left(M X_{\varsigma}^{(i)}\right)\left[\tilde{Y}_{q}\right]\right|_{s^{\prime}, r^{\prime}} \leq\left|d\left(M X_{\varsigma}\right)\left[\tilde{Y}_{q}\right]\right|_{s^{\prime}, r^{\prime}}
$$


and

$$
\begin{aligned}
&\left|d\left(M X_{\varsigma}\right)\left[\tilde{Y}_{q}\right]\right|_{s^{\prime}, r^{\prime}} \stackrel{(2.53)}{=} \sup _{v \in D\left(s^{\prime}, r^{\prime}\right)}\left\|d\left(M X_{\varsigma}\right)(v)\left[\tilde{Y}_{q}(v)\right]\right\|_{E, s^{\prime}, r^{\prime}} \\
& \leq \sup _{v \in D\left(s^{\prime}, r^{\prime}\right)}\left\|d\left(M X_{\varsigma}\right)(v)\right\|_{\mathcal{L}\left((E, s, r),\left(E, s^{\prime}, r^{\prime}\right)\right)}\left\|\tilde{Y}_{q}(v)\right\|_{E, s, r} \\
& \stackrel{(2.65)}{\leq} 4 \delta^{-1}\left|M X_{\varsigma}\right|_{s, r} \sup _{v \in D\left(s^{\prime}, r^{\prime}\right)}\left\|\tilde{Y}_{q}(v)\right\|_{E, s, r} \\
& \stackrel{(2.62),(2.74)}{\leq} 4 \delta^{-1}\left\|X_{\varsigma}\right\|_{s, r} \sup _{v \in D\left(s^{\prime}, r^{\prime}\right)}\|(M Y)(v)\|_{E, s, r} \\
& \quad \stackrel{(2.53)}{\leq} 4 \delta^{-1}\left\|X_{\varsigma}\right\|_{s, r}|M Y|_{s, r} \stackrel{(2.64)}{\leq} 4 \delta^{-1}\left\|X_{\varsigma}\right\|_{s, r}\|Y\|_{s, r}<+\infty
\end{aligned}
$$

because $\|Y\|_{s, r}<+\infty$ and $\left\|X_{\varsigma}\right\|_{s, r} \leq\|X\|_{s, r}<+\infty$ by assumption.

Hence Lemma 2.6 implies

$$
d X_{\varsigma}^{(i)}[Y] \stackrel{(2.47)}{=} \sum_{j}\left(\partial_{j} X_{\varsigma}^{(i)}\right) Y^{(j)}=: f \stackrel{\text { Lemma } 2.6}{\prec} g:=d\left(M X_{\varsigma}^{(i)}\right)\left[\tilde{Y}_{q}\right], \quad \forall i \in \mathcal{J},
$$

namely, by (2.37) and Definition 2.7,

$$
d X_{\varsigma}[Y] \prec M\left(d X_{\varsigma}[Y]\right) \prec d\left(M X_{\varsigma}\right)\left[\tilde{Y}_{q}\right] .
$$

Hence (2.73) is fully justified. By (2.77) and (2.63) we get

$$
\begin{aligned}
\left\|d X_{\varsigma}[Y]\right\|_{s^{\prime}, r^{\prime}} \leq\left\|d\left(M X_{\varsigma}\right)\left[\tilde{Y}_{q}\right]\right\|_{s^{\prime}, r^{\prime}} & \stackrel{(2.64)}{\leq} 2^{n}\left|M\left(d\left(M X_{\varsigma}\right)\left[\tilde{Y}_{q}\right]\right)\right|_{s^{\prime}, r^{\prime}} \\
& =2^{n}\left|d\left(M X_{\varsigma}\right)\left[\tilde{Y}_{q}\right]\right|_{s^{\prime}, r^{\prime}}
\end{aligned}
$$

because $d\left(M X_{\varsigma}\right)\left[\tilde{Y}_{q}\right]$ coincides with its majorant by (2.77). Finally (2.70) follows by (2.78), (2.76).

\subsection{Hamiltonian formalism}

Given a function $H: D(s, r) \subset E \rightarrow \mathbb{C}$ we define the associated Hamiltonian vector field

$$
X_{H}:=\left(\partial_{y} H,-\partial_{x} H,-\mathrm{i} \partial_{\bar{z}} H, \mathrm{i} \partial_{z} H\right)
$$

where the partial derivatives are defined as in (2.30).

For a subset of indices $I \subset \mathbb{I}$, the bound (2.55) implies

$$
\left\|X_{\Pi_{I} H}\right\|_{s, r} \leq\left\|X_{H}\right\|_{s, r} .
$$

The Poisson brackets are defined by

$$
\begin{aligned}
\{H, K\} & :=\{H, K\}^{x, y}+\{H, K\}^{z, \bar{z}} \\
& :=\left(\partial_{x} H \cdot \partial_{y} K-\partial_{x} K \cdot \partial_{y} H\right)+\mathrm{i}\left(\partial_{z} H \cdot \partial_{\bar{z}} K-\partial_{\bar{z}} H \cdot \partial_{z} K\right) \\
& =\partial_{x} H \cdot \partial_{y} K-\partial_{x} K \cdot \partial_{y} H+\mathrm{i} \partial_{z^{+}} H \cdot \partial_{z^{-}} K-\mathrm{i} \partial_{z^{-}} H \cdot \partial_{z^{+}} K \\
& =\partial_{x} H \cdot \partial_{y} K-\partial_{x} K \cdot \partial_{y} H+\mathrm{i} \sum_{\sigma= \pm, j \in \mathbb{Z} \backslash \mathcal{I}} \sigma \partial_{z_{j}^{\sigma}} H \partial_{z_{j}^{-\sigma}} K
\end{aligned}
$$


where "." denotes the standard pairing $a \cdot b:=\sum_{j} a_{j} b_{j}$. We recall the Jacobi identity

$$
\{\{K, G\}, H\}+\{\{G, H\}, K\}+\{\{H, K\}, G\}=0 .
$$

Along this paper we shall use the Lie algebra notations

$$
\operatorname{ad}_{F}:=\{, F\}, \quad e^{\operatorname{ad}_{F}}:=\sum_{k=0}^{\infty} \frac{\operatorname{ad}_{F}^{k}}{k !} .
$$

Given a set of indices

$$
\mathcal{I}:=\left\{\mathrm{j}_{1}, \ldots, \mathrm{j}_{n}\right\} \subset \mathbb{Z}
$$

we define the momentum

$$
\mathcal{M}:=\mathcal{M}_{\mathcal{I}}:=\sum_{l=1}^{n} \mathrm{j}_{l} y_{l}+\sum_{j \in \mathbb{Z} \backslash \mathcal{I}} j z_{j} \bar{z}_{j}=\sum_{l=1}^{n} \mathrm{j}_{l} y_{l}+\sum_{j \in \mathbb{Z} \backslash \mathcal{I}} j z_{j}^{+} z_{j}^{-} .
$$

We say that a function $H$ satisfies momentum conservation if $\{H, \mathcal{M}\}=0$.

By (2.81), any monomial $e^{\mathrm{i} k \cdot x} y^{i} z^{\alpha} \bar{z}^{\beta}$ is an eigenvector of the operator ad ${ }_{\mathcal{M}}$, namely

$$
\left\{e^{\mathrm{i} k \cdot x} y^{i} z^{\alpha} \bar{z}^{\beta}, \mathcal{M}\right\}=\pi(k, \alpha, \beta) e^{\mathrm{i} k \cdot x} y^{i} z^{\alpha} \bar{z}^{\beta}
$$

where

$$
\pi(k, \alpha, \beta):=\sum_{l=1}^{n} j_{l} k_{l}+\sum_{j \in \mathbb{Z} \backslash \mathcal{I}} j\left(\alpha_{j}-\beta_{j}\right) .
$$

We refer to $\pi(k, \alpha, \beta)$ as the momentum of the monomial $e^{\mathrm{i} k \cdot x} y^{i} z^{\alpha} \bar{z}^{\beta}$. A monomial satisfies momentum conservation if and only if $\pi(k, \alpha, \beta)=0$. Moreover, a power series (2.7) with $\|f\|_{s, r}<+\infty$ satisfies momentum conservation if and only if all its monomials have zero momentum.

Let $\mathcal{O} \subset \mathbb{R}^{n}$ be a subset of parameters, and

$$
f: D(s, r) \times \mathcal{O} \rightarrow \mathbb{C} \quad \text { with } \quad X_{f}: D(s, r) \times \mathcal{O} \rightarrow E .
$$

For $\lambda>0$, we consider

$$
\begin{aligned}
\left|X_{f}\right|_{s, r, \mathcal{O}}^{\lambda}:=\left|X_{f}\right|_{s, r}^{\lambda} & :=\sup _{\mathcal{O}}\left|X_{f}\right|_{s, r}+\lambda\left|X_{f}\right|_{s, r}^{\operatorname{lip}} \\
& :=\sup _{\xi \in \mathcal{O}}\left|X_{f}(\xi)\right|_{s, r}+\lambda \sup _{\xi, \eta \in \mathcal{O}, \xi \neq \eta} \frac{\left|X_{f}(\xi)-X_{f}(\eta)\right|_{s, r}}{|\xi-\eta|} .
\end{aligned}
$$

Note that $|\cdot|_{s, r}^{\lambda}$ is only a semi-norm on spaces of functions $f$ because the Hamiltonian vector field $X_{f}=0$ when $f$ is constant.

Definition 2.8. - A function $f$ as in (2.87) is called

- regular, if the sup-norm $\left|X_{f}\right|_{s, r, \mathcal{O}}:=\sup _{\mathcal{O}}\left|X_{f}\right|_{s, r}<\infty$, see (2.53).

- M-regular, if the majorant norm $\left\|X_{f}\right\|_{s, r, \mathcal{O}}:=\sup _{\mathcal{O}}\left\|X_{f}\right\|_{s, r}<\infty$, see (2.54).

- $\lambda$-regular, if the Lipschitz semi-norm $\left|X_{f}\right|_{s, r, \mathcal{O}}^{\lambda}<\infty$, see (2.88). 
We denote by $\mathcal{H}_{s, r}$ the space of $M$-regular Hamiltonians and by $\mathcal{H}_{s, r}^{\text {null }}$ its subspace of functions satisfying momentum conservation.

When $\mathcal{I}=\varnothing$ (namely there are no $(x, y)$-variables) we denote the space of M-regular functions simply by $\mathcal{H}_{r}$, similarly $\mathcal{H}_{r}^{\text {null }}$, and we drop s form the norms, i.e., $|\cdot|_{r},\|\cdot\|_{r},|\cdot|_{r, \mathcal{O}}$, etc.

Note that, by (2.62) and (2.88), we have

$$
\mathrm{M}-\text { regular } \Longrightarrow \text { regular } \Longleftarrow \lambda \text {-regular . }
$$

If $H, F$ satisfy momentum conservation, the same holds for $\{H, K\}$. Indeed by the Jacobi identity (2.82),

$$
\{\mathcal{M}, H\}=0 \text { and }\{\mathcal{M}, K\}=0 \quad \Longrightarrow \quad\{\mathcal{M},\{H, K\}\}=0 .
$$

For $H, K \in \mathcal{H}_{s, r}$ we have

$$
X_{\{H, K\}}=d X_{H}\left[X_{K}\right]-d X_{K}\left[X_{H}\right]=\left[X_{H}, X_{K}\right]
$$

and the commutator Lemma 2.15 implies the fundamental lemma below.

Lemma 2.16. - Let $H, K \in \mathcal{H}_{s, r}$. Then, for all $r / 2 \leq r^{\prime}<r, s / 2 \leq s^{\prime}<s$

$$
\left\|X_{\{H, K\}}\right\|_{s^{\prime}, r^{\prime}}=\left\|\left[X_{H}, X_{K}\right]\right\|_{s^{\prime}, r^{\prime}} \leq 2^{2 n+3} \delta^{-1}\left\|X_{H}\right\|_{s, r}\left\|X_{K}\right\|_{s, r}
$$

where $\delta$ is defined in (2.66).

Unlike the sup-norm, the majorant norm of a function is very sensitive to coordinate transformations. For our purposes, we only need to consider close to identity canonical transformations that are generated by an $M$-regular Hamiltonian flow. We show below that the $M$-regular functions are closed under this group and we estimate the majorant norm of the transformed Hamiltonian vector field.

Lemma 2.17 (Hamiltonian flow). - Let $r / 2 \leq r^{\prime}<r, s / 2 \leq s^{\prime}<s$, and $F \in \mathcal{H}_{s, r}$ with

$$
\left\|X_{F}\right\|_{s, r}<\eta:=\delta /\left(2^{2 n+5} e\right)
$$

with $\delta$ defined in (2.66). Then the time 1-hamiltonian flow

$$
\Phi_{F}^{1}: D\left(s^{\prime}, r^{\prime}\right) \rightarrow D(s, r)
$$

is well defined, analytic, symplectic, and, $\forall H \in \mathcal{H}_{s, r}$, we have $H \circ \Phi_{F}^{1} \in \mathcal{H}_{s^{\prime}, r^{\prime}}$ and

$$
\left\|X_{H \circ \Phi_{F}^{1}}\right\|_{s^{\prime}, r^{\prime}} \leq \frac{\left\|X_{H}\right\|_{s, r}}{1-\eta^{-1}\left\|X_{F}\right\|_{s, r}} .
$$

Finally if $F, H \in \mathcal{H}_{s, r}^{\text {null }}$ then $H \circ \Phi_{F}^{1} \in \mathcal{H}_{s^{\prime}, r^{\prime}}^{\text {null }}$.

Proof. - We estimate by Lie series the Hamiltonian vector field of

$$
H^{\prime}=H \circ \Phi_{F}^{1}=e^{\operatorname{ad}_{\mathrm{F}}} H=\sum_{k=0}^{\infty} \frac{\operatorname{ad}_{F}^{k} H}{k !}=\sum_{k=0}^{\infty} \frac{H^{(k)}}{k !}, \quad \text { i.e., } X_{H^{\prime}}=\sum_{k=0}^{\infty} \frac{X_{H^{(k)}}}{k !},
$$

where $H^{(i)}:=\operatorname{ad}_{F}^{i}(H)=\operatorname{ad}_{F}\left(H^{(i-1)}\right), H^{(0)}:=H$.

For each $k \geq 0$, divide the intervals $\left[s^{\prime}, s\right]$ and $\left[r^{\prime}, r\right]$ into $k$ equal segments and set

$$
s_{i}:=s-i \frac{s-s^{\prime}}{k}, \quad r_{i}:=r-i \frac{r-r^{\prime}}{k}, \quad i=0, \ldots, k .
$$


By (2.92) we have

$$
\left\|X_{H^{(i)}}\right\|_{s_{i}, r_{i}}=\left\|\left[X_{F}, X_{H^{(i-1)}}\right]\right\|_{s_{i}, r_{i}} \leq 2^{2 n+3} \delta_{i}^{-1}\left\|X_{H^{(i-1)}}\right\|_{s_{i-1}, r_{i-1}}\left\|X_{F}\right\|_{s_{i-1}, r_{i-1}}
$$

where

$$
\delta_{i}:=\min \left\{1-\frac{s_{i}}{s_{i-1}}, 1-\frac{r_{i}}{r_{i-1}}\right\} \geq \frac{\delta}{k} .
$$

By (2.96)-(2.97) we deduce

$$
\left\|X_{H^{(i)}}\right\|_{s_{i}, r_{i}} \leq 2^{2 n+3} k \delta^{-1}\left\|X_{H^{(i-1)}}\right\|_{s_{i-1}, r_{i-1}}\left\|X_{F}\right\|_{s_{i-1}, r_{i-1}}, \quad i=1, \ldots, k .
$$

Iterating $k$-times, and using $\left\|X_{F}\right\|_{s_{i-1}, r_{i-1}} \leq 4\left\|X_{F}\right\|_{s, r}$ (see (2.3))

$$
\left\|X_{H^{(k)}}\right\|_{s^{\prime}, r^{\prime}} \leq\left(2^{2 n+5} k \delta^{-1}\right)^{k}\left\|X_{H}\right\|_{s, r}\left\|X_{F}\right\|_{s, r}^{k} .
$$

By (2.95), using $k^{k} \leq e^{k} k$ ! and recalling the definition of $\eta$ in (2.93), we estimate

$$
\begin{aligned}
\left\|X_{H^{\prime}}\right\|_{s^{\prime}, r^{\prime}} & \stackrel{(2.95)}{\leq} \sum_{k=0}^{\infty} \frac{\left\|X_{H^{(k)}}\right\|_{s^{\prime}, r^{\prime}}}{k !} \stackrel{(2.98)}{\leq}\left\|X_{H}\right\|_{s, r} \sum_{k=0}^{\infty} \frac{\left(2^{2 n+5} k \delta^{-1}\left\|X_{F}\right\|_{s, r}\right)^{k}}{k !} \\
& \leq\left\|X_{H}\right\|_{s, r} \sum_{k=0}^{\infty}\left(\eta^{-1}\left\|X_{F}\right\|_{s, r}\right)^{k} \stackrel{(2.93)}{=} \frac{\left\|X_{H}\right\|_{s, r}}{1-\eta^{-1}\left\|X_{F}\right\|_{s, r}}
\end{aligned}
$$

proving (2.94).

Finally, if $F$ and $H$ satisfy momentum conservation then each $\operatorname{ad}_{F}^{k} H, k \geq 1$, satisfies momentum conservation. For $k=1$ it is proved in (2.90) and, for $k>1$, it follows by induction and the Jacobi identity (2.82). By (2.95) we conclude that also $H \circ \Phi_{F}^{1}$ satisfies momentum conservation.

We conclude this section with two simple lemmata.

Lemma 2.18. - Let $P=\sum_{|k| \leq K, i, \alpha, \beta} P_{k, i, \alpha, \beta} e^{\mathrm{i} k \cdot x} y^{i} z^{\alpha} \bar{z}^{\beta}$ and $\left|\Delta_{k, i, \alpha, \beta}\right| \geq \gamma\langle k\rangle^{-\tau}$, $\forall|k| \leq K, i, \alpha, \beta$. Then

$$
F:=\sum_{|k| \leq K, i, \alpha, \beta} \frac{P_{k, i, \alpha, \beta}}{\Delta_{k, i, \alpha, \beta}} e^{\mathrm{i} k \cdot x} y^{i} z^{\alpha} \bar{z}^{\beta} \quad \text { satisfies } \quad\left\|X_{F}\right\|_{s, r} \leq \gamma^{-1} K^{\tau}\left\|X_{P}\right\|_{s, r} .
$$

Proof. - By Definition 2.6 and $\left|\Delta_{k, i, \alpha, \beta}\right| \geq \gamma K^{-\tau}$ for all $|k| \leq K$.

Lemma 2.19. - Let $P=\sum_{j \in \mathbb{Z} \backslash \mathcal{I}} P_{j} z_{j} \bar{z}_{j}$ with $\left\|X_{P}\right\|_{r}<\infty$. Then $\left|P_{j}\right| \leq\left\|X_{P}\right\|_{r}$.

Proof. - By (2.79) and Definition 2.6 we have

$$
\left\|X_{P}\right\|_{r}^{2}=2 \sup _{\|z\|_{a, p}<r} \sum_{h \in \mathbb{Z} \backslash \mathcal{I}}\left|P_{h}\right|^{2} \frac{\left|z_{h}\right|^{2}}{r^{2}} e^{2 a|h|}\langle h\rangle^{2 p} \geq\left|P_{j}\right|^{2}
$$

by evaluating at $z_{h}^{(j)}:=\delta_{j h} e^{-a|j|}\langle j\rangle^{p} r / \sqrt{2}$. 


\section{Quasi-Töplitz functions}

Let $N_{0} \in \mathbb{N}, \theta, \mu \in \mathbb{R}$ be parameters such that

$$
1<\theta, \mu<6, \quad 12 N_{0}^{L-1}+2 \kappa N_{0}^{b-1}<1, \quad \kappa:=\max _{1 \leq l \leq n}\left|j_{l}\right|,
$$

(the $j_{l}$ are defined in (2.84)) where

$$
0<b<L<1
$$

For $N \geq N_{0}$, we decompose

$$
\ell_{\mathcal{I}}^{a, p} \times \ell_{\mathcal{I}}^{a, p}=\ell_{L}^{a, p} \oplus \ell_{R}^{a, p} \oplus \ell_{H}^{a, p}
$$

where

$$
\begin{gathered}
\ell_{L}^{a, p}:=\ell_{L}^{a, p}(N):=\left\{w=\left(z^{+}, z^{-}\right) \in \ell_{\mathcal{I}}^{a, p} \times \ell_{\mathcal{I}}^{a, p}: z_{j}^{\sigma}=0, \sigma= \pm, \forall|j| \geq 6 N^{L}\right\} \\
\ell_{R}^{a, p}:=\ell_{R}^{a, p}(N):=\left\{w=\left(z^{+}, z^{-}\right) \in \ell_{\mathcal{I}}^{a, p} \times \ell_{\mathcal{I}}^{a, p}: z_{j}^{\sigma}=0, \sigma= \pm, \text { unless } 6 N^{L}<|j|<N\right\} \\
\ell_{H}^{a, p}:=\ell_{H}^{a, p}(N):=\left\{w=\left(z^{+}, z^{-}\right) \in \ell_{\mathcal{I}}^{a, p} \times \ell_{\mathcal{I}}^{a, p}: z_{j}^{\sigma}=0, \sigma= \pm, \forall|j| \leq N\right\} .
\end{gathered}
$$

Note that by (3.1)-(3.2) the subspaces $\ell_{L}^{a, p} \cap \ell_{H}^{a, p}=0$ and $\ell_{R}^{a, p} \neq 0$. Accordingly we decompose any

$$
w \in \ell^{a, p} \times \ell^{a, p} \quad \text { as } \quad w=w_{L}+w_{R}+w_{H}
$$

and we call $w_{L} \in \ell_{L}^{a, p}$ the "low momentum variables" and $w_{H} \in \ell_{H}^{a, p}$ the "high momentum variables".

We split the Poisson brackets in (2.81) as

$$
\{\cdot, \cdot\}=\{\cdot, \cdot\}^{x, y}+\{\cdot, \cdot\}^{L}+\{\cdot, \cdot\}^{R}+\{\cdot, \cdot\}^{H}
$$

where

$$
\{H, K\}^{H}:=\mathrm{i} \sum_{\sigma= \pm,|j|>c N} \sigma \partial_{z_{j}^{\sigma}} H \partial_{z_{j}^{-\sigma}} K
$$

The other Poisson brackets $\{\cdot, \cdot\}^{L},\{\cdot, \cdot\}^{R}$ are defined analogously with respect to the splitting (3.3).

\section{Lemma 3.1. - Consider two monomials}

$$
\mathfrak{m}=c_{k, i, \alpha, \beta} e^{\mathrm{i} k \cdot x} y^{i} z^{\alpha} \bar{z}^{\beta} \text { and } \mathfrak{m}^{\prime}=c_{k^{\prime}, i^{\prime}, \alpha^{\prime}, \beta^{\prime}}^{\prime} e^{\mathrm{i} k^{\prime} \cdot x} y^{i^{\prime}} z^{\alpha^{\prime}} \bar{z}^{\beta^{\prime}}
$$

The momentum of $\mathfrak{m m}^{\prime},\left\{\mathfrak{m}, \mathfrak{m}^{\prime}\right\},\left\{\mathfrak{m}, \mathfrak{m}^{\prime}\right\}^{x, y},\left\{\mathfrak{m}, \mathfrak{m}^{\prime}\right\}^{L},\left\{\mathfrak{m}, \mathfrak{m}^{\prime}\right\}^{R},\left\{\mathfrak{m}, \mathfrak{m}^{\prime}\right\}^{H}$, equals the sum of the momenta of each monomial $\mathfrak{m}, \mathfrak{m}^{\prime}$.

Proof. - By (2.86), (2.81), and $\pi\left(k+k^{\prime}, \alpha+\alpha^{\prime}, \beta+\beta^{\prime}\right)=\pi(k, \alpha, \beta)+\pi\left(k^{\prime}, \alpha^{\prime}, \beta^{\prime}\right)=\pi\left(k, \alpha-e_{j}, \beta\right)+\pi\left(k^{\prime}, \alpha^{\prime}, \beta^{\prime}-e_{j}\right)$, for any $j \in \mathbb{Z}$.

We now define subspaces of $\mathcal{H}_{s, r}$ (recall Definition 2.8). 
Definition 3.1. - Low-momentum A monomial $e^{\mathrm{i} k \cdot x} y^{i} z^{\alpha} \bar{z}^{\beta}$ is $(N, \mu)$-low momentum if

$$
\sum_{j \in \mathbb{Z} \backslash \mathcal{I}}|j|\left(\alpha_{j}+\beta_{j}\right)<\mu N^{L}, \quad|k|<N^{b} .
$$

We denote by

$$
\mathcal{L}_{s, r}(N, \mu) \subset \mathcal{H}_{s, r}
$$

the subspace of functions

$$
g=\sum g_{k, i, \alpha, \beta} e^{\mathrm{i} k \cdot x} y^{i} z^{\alpha} \bar{z}^{\beta} \in \mathcal{H}_{s, r}
$$

whose monomials are $(N, \mu)$-low momentum. The corresponding projection

$$
\Pi_{N, \mu}^{L}: \mathcal{H}_{s, r} \rightarrow \mathcal{L}_{s, r}(N, \mu)
$$

is defined as $\Pi_{N, \mu}^{L}:=\Pi_{I}$ (see (2.13)) where $I$ is the subset of $\mathbb{I}$ (see (2.8)) satisfying (3.5). Finally, given $h \in \mathbb{Z}$, we denote by

$$
\mathcal{L}_{s, r}(N, \mu, h) \subset \mathcal{L}_{s, r}(N, \mu)
$$

the subspace of functions whose monomials satisfy

$$
\pi(k, \alpha, \beta)+h=0 .
$$

By (3.5), (3.1)-(3.2), any function in $\mathcal{L}_{s, r}(N, \mu), 1<\mu<6$, only depends on $x, y, w_{L}$ and therefore

$$
g, g^{\prime} \in \mathcal{L}_{s, r}(N, \mu) \Longrightarrow g g^{\prime},\left\{g, g^{\prime}\right\}^{x, y},\left\{g, g^{\prime}\right\}^{L} \text { do not depend on } w_{H}
$$

Moreover, by (2.86), (3.1), (3.5), if

$$
|h| \geq \mu N^{L}+\kappa N^{b} \quad \Longrightarrow \quad \mathcal{L}_{s, r}(N, \mu, h)=\varnothing .
$$

Definition $3.2((N, \theta, \mu)$-bilinear $)$. - We denote by

$$
\mathcal{B}_{s, r}(N, \theta, \mu) \subset \mathcal{H}_{s, r}^{\text {null }}
$$

the subspace of the $(N, \theta, \mu)$-bilinear functions defined as

(3.11) $f:=\sum_{|m|,|n|>\theta N, \sigma, \sigma^{\prime}= \pm} f_{m, n}^{\sigma, \sigma^{\prime}}\left(x, y, w_{L}\right) z_{m}^{\sigma} z_{n}^{\sigma^{\prime}} \quad$ with $\quad f_{m, n}^{\sigma, \sigma^{\prime}} \in \mathcal{L}_{s, r}\left(N, \mu, \sigma m+\sigma^{\prime} n\right)$

and we denote the projection

$$
\Pi_{N, \theta, \mu}: \mathcal{H}_{s, r} \rightarrow \mathcal{B}_{s, r}(N, \theta, \mu) .
$$

Explicitely, for $g \in \mathcal{H}_{s, r}$ as in (3.6), the coefficients in (3.11) of $f:=\Pi_{N, \theta, \mu} g$ are

$$
f_{m, n}^{\sigma, \sigma^{\prime}}\left(x, y, w^{L}\right):=\sum_{\substack{(k, i, \alpha, \beta) \text { s.t. (3.5) holds } \\ \text { and } \pi(k, \alpha, \beta)=-\sigma m-\sigma^{\prime} n}} f_{k, i, \alpha, \beta, m, n}^{\sigma, \sigma^{\prime}} e^{\mathrm{i} k \cdot x} y^{i} z^{\alpha} \bar{z}^{\beta}
$$

where

$$
\begin{array}{ll}
f_{k, i, \alpha, \beta, m, n}^{+,+}:=\left(2-\delta_{m n}\right)^{-1} g_{k, i, \alpha+e_{m}+e_{n}, \beta}, & f_{k, i, \alpha, \beta, m, n}^{+,-}:=g_{k, i, \alpha+e_{m}, \beta+e_{n}}, \\
f_{k, i, \alpha, \beta, m, n}^{-,-}:=\left(2-\delta_{m n}\right)^{-1} g_{k, i, \alpha, \beta+e_{m}+e_{n}}, & f_{k, i, \alpha, \beta, m, n}^{-,+}:=g_{k, i, \alpha+e_{n}, \beta+e_{m}} .
\end{array}
$$

For parameters $1<\theta<\theta^{\prime}, 6>\mu>\mu^{\prime}$, we have

$$
\mathcal{B}_{s, r}\left(N, \theta^{\prime}, \mu^{\prime}\right) \subset \mathcal{B}_{s, r}(N, \theta, \mu) .
$$


Remark 3.1. - The projection $\Pi_{N, \theta, \mu}$ can be written in the form $\Pi_{I}$, see (2.13), for a suitable $I \subset \mathbb{I}$. The representation in (3.11) is not unique. It becomes unique if we impose the "symmetric" conditions

$$
f_{m, n}^{\sigma, \sigma^{\prime}}=f_{n, m}^{\sigma^{\prime}, \sigma}
$$

Note that the coefficients in (3.12)-(3.13) satisfy (3.14).

\subsection{Töplitz functions}

Let $N \geq N_{0}$.

Definition 3.3 (Töplitz). - A function $f \in \mathcal{B}_{s, r}(N, \theta, \mu)$ is $(N, \theta, \mu)$-Töplitz if the coefficients in (3.11) have the form

$$
f_{m, n}^{\sigma, \sigma^{\prime}}=f^{\sigma, \sigma^{\prime}}\left(\mathbf{s}(m), \sigma m+\sigma^{\prime} n\right) \quad \text { for some } f^{\sigma, \sigma^{\prime}}(\varsigma, h) \in \mathcal{L}_{s, r}(N, \mu, h),
$$

with $\mathbf{s}(m):=\operatorname{sign}(m), \varsigma=+,-$ and $h \in \mathbb{Z}$. We denote by

$$
\mathcal{T}_{s, r}:=\mathcal{T}_{s, r}(N, \theta, \mu) \subset \mathcal{B}_{s, r}(N, \theta, \mu)
$$

the space of the $(N, \theta, \mu)$-Töplitz functions.

For parameters $N^{\prime} \geq N, \theta^{\prime} \geq \theta, \mu^{\prime} \leq \mu, r^{\prime} \leq r, s^{\prime} \leq s$ we have

$$
\mathcal{T}_{s, r}(N, \theta, \mu) \subseteq \mathcal{T}_{s^{\prime}, r^{\prime}}\left(N^{\prime}, \theta^{\prime}, \mu^{\prime}\right) .
$$

Lemma 3.2. - Consider $f, g \in \mathcal{T}_{s, r}(N, \theta, \mu)$ and $p \in \mathcal{L}_{s, r}\left(N, \mu_{1}, 0\right)$ with $1<\mu, \mu_{1}<6$. For all $0<s^{\prime}<s, 0<r^{\prime}<r$ and $\theta^{\prime} \geq \theta, \mu^{\prime} \leq \mu$ one has

$$
\Pi_{N, \theta^{\prime}, \mu^{\prime}}\{f, p\}^{L}, \Pi_{N, \theta^{\prime}, \mu^{\prime}}\{f, p\}^{x, y} \in \mathcal{T}_{s^{\prime}, r^{\prime}}\left(N, \theta^{\prime}, \mu^{\prime}\right) .
$$

If moreover

$$
\mu N^{L}+\kappa N^{b}<\left(\theta^{\prime}-\theta\right) N
$$

then

$$
\Pi_{N, \theta^{\prime}, \mu^{\prime}}\{f, g\}^{H} \in \mathcal{T}_{s^{\prime}, r^{\prime}}\left(N, \theta^{\prime}, \mu^{\prime}\right) .
$$

Proof. - Write $f \in \mathcal{T}_{s, r}(N, \theta, \mu)$ as in (3.11) where $f_{m, n}^{\sigma, \sigma^{\prime}}$ satisfy (3.15) and (3.14), namely

$$
f_{m, n}^{\sigma, \sigma^{\prime}}=f_{n, m}^{\sigma^{\prime}, \sigma}=f^{\sigma, \sigma^{\prime}}\left(\mathbf{s}(m), \sigma m+\sigma^{\prime} n\right) \in \mathcal{L}_{s, r}\left(N, \mu, \sigma m+\sigma^{\prime} n\right),
$$

similarly for $g$.

Proof of (3.17). - Since the variables $z_{m}^{\sigma}, z_{n}^{\sigma^{\prime}},|m|,|n|>\theta N$, are high momentum,

$$
\left\{f_{m, n}^{\sigma, \sigma^{\prime}} z_{m}^{\sigma} z_{n}^{\sigma^{\prime}}, p\right\}^{L}=\left\{f_{m, n}^{\sigma, \sigma^{\prime}}, p\right\}^{L} z_{m}^{\sigma} z_{n}^{\sigma^{\prime}}
$$

and $\left\{f_{m, n}^{\sigma, \sigma^{\prime}}, p\right\}^{L}$ does not depend on $w_{H}$ by (3.9) (recall that $f_{m, n}^{\sigma, \sigma^{\prime}}, p \in \mathcal{L}_{s, r}(N, \mu)$ ). The coefficient of $z_{m}^{\sigma} z_{n}^{\sigma^{\prime}}$ in $\Pi_{N, \theta^{\prime}, \mu^{\prime}}\{f, p\}^{L}$ is

$$
\Pi_{N, \mu^{\prime}}^{L}\left\{f_{m, n}^{\sigma, \sigma^{\prime}}, p\right\}^{L} \stackrel{(3.20)}{=} \Pi_{N, \mu^{\prime}}^{L}\left\{f^{\sigma, \sigma^{\prime}}\left(\mathbf{s}(m), \sigma m+\sigma^{\prime} n\right), p\right\}^{L} \in \mathcal{L}_{s^{\prime}, r^{\prime}}\left(N, \mu^{\prime}, \sigma m+\sigma^{\prime} n\right)
$$

using Lemma 3.1 (recall that $p$ has zero momentum). The proof that $\Pi_{N, \theta^{\prime}, \mu^{\prime}}\{f, p\}^{x, y} \in$ $\mathcal{T}_{s^{\prime}, r^{\prime}}\left(N, \theta^{\prime}, \mu^{\prime}\right)$ is analogous. 
Proof of (3.19). - A direct computation, using (3.4), gives

$$
\{f, g\}^{H}=\sum_{|m|,|n|>\theta N, \sigma, \sigma^{\prime}= \pm} p_{m, n}^{\sigma, \sigma^{\prime}} z_{m}^{\sigma} z_{n}^{\sigma^{\prime}}
$$

with

$$
p_{m, n}^{\sigma, \sigma^{\prime}}=2 \mathrm{i} \sum_{|l|>\theta N, \sigma_{1}= \pm} \sigma_{1}\left(f_{m, l}^{\sigma, \sigma_{1}} g_{l, n}^{-\sigma_{1}, \sigma^{\prime}}+f_{n, l}^{\sigma^{\prime}, \sigma_{1}} g_{l, m}^{-\sigma_{1}, \sigma}\right) .
$$

By (3.9) the coefficient $p_{m, n}^{\sigma, \sigma^{\prime}}$ does not depend on $w_{H}$. Therefore

$$
\Pi_{N, \theta^{\prime}, \mu^{\prime}}\{f, g\}^{H}=\sum_{|m|,|n|>\theta^{\prime} N, \sigma, \sigma^{\prime}= \pm} q_{m, n}^{\sigma, \sigma^{\prime}} z_{m}^{\sigma} z_{n}^{\sigma^{\prime}} \quad \text { with } \quad q_{m, n}^{\sigma, \sigma^{\prime}}:=\Pi_{N, \mu^{\prime}}^{L} p_{m, n}^{\sigma, \sigma^{\prime}}
$$

(recall (3.7)). It results $q_{m, n}^{\sigma, \sigma^{\prime}} \in \mathcal{L}_{s^{\prime}, r^{\prime}}\left(N, \mu^{\prime}, \sigma m+\sigma^{\prime} n\right)$ by (3.22), (3.21), and Lemma 3.1 since, i.e.,

$$
f_{m, l}^{\sigma, \sigma_{1}} \in \mathcal{L}_{s, r}\left(N, \mu, \sigma m+\sigma_{1} l\right) \quad \text { and } \quad g_{l, n}^{-\sigma_{1}, \sigma^{\prime}} \in \mathcal{L}_{s, r}\left(N, \mu,-\sigma_{1} l+\sigma^{\prime} n\right) .
$$

Hence the $\left(N, \theta^{\prime}, \mu^{\prime}\right)$-bilinear function $\Pi_{N, \theta^{\prime}, \mu^{\prime}}\{f, g\}^{H}$ in (3.22) is written in the form (3.11). It remains to prove that it is $\left(N, \theta^{\prime}, \mu^{\prime}\right)$-Töplitz, namely that for all $|m|,|n|>\theta^{\prime} N, \sigma, \sigma^{\prime}= \pm$,

$$
q_{m, n}^{\sigma, \sigma^{\prime}}=q^{\sigma, \sigma^{\prime}}\left(\mathrm{s}(m), \sigma m+\sigma^{\prime} n\right) \quad \text { for some } \quad q^{\sigma, \sigma^{\prime}}(\varsigma, h) \in \mathcal{L}_{s, r}\left(N, \mu^{\prime}, h\right) .
$$

Let us consider in (3.21)-(3.22) the term (with $m, n, \sigma, \sigma^{\prime}, \sigma_{1}$ fixed)

$$
\Pi_{N, \mu^{\prime}}^{L} \sum_{|l|>\theta N} f_{m, l}^{\sigma, \sigma_{1}} g_{l, n}^{-\sigma_{1}, \sigma^{\prime}}
$$

(the other is analogous). Since $f, g \in \mathcal{T}_{s, r}(N, \theta, \mu)$ we have

$$
\begin{gathered}
f_{m, l}^{\sigma, \sigma_{1}}=f^{\sigma, \sigma_{1}}\left(\mathrm{~s}(m), \sigma m+\sigma_{1} l\right) \in \mathcal{L}_{s, r}\left(N, \mu, \sigma m+\sigma_{1} l\right) \\
g_{l, n}^{-\sigma_{1}, \sigma^{\prime}}=g^{-\sigma_{1}, \sigma^{\prime}}\left(\mathbf{s}(l),-\sigma_{1} l+\sigma^{\prime} n\right) \in \mathcal{L}_{s, r}\left(N, \mu,-\sigma_{1} l+\sigma^{\prime} n\right) .
\end{gathered}
$$

By (3.10), (3.25), (3.26), if the coefficients $f_{m, l}^{\sigma, \sigma_{1}}, g_{l, n}^{-\sigma_{1}, \sigma^{\prime}}$ are not zero then

$$
\left|\sigma m+\sigma_{1} l\right|,\left|-\sigma_{1} l+\sigma^{\prime} n\right|<\mu N^{L}+\kappa N^{b} .
$$

By (3.27), (3.1), we get $c N>\left|\sigma m+\sigma_{1} l\right|=\left|\sigma \sigma_{1} \mathbf{s}(m)\right| m|+\mathbf{s}(l)| l||$, which implies, since $|m|>\theta^{\prime} N>N$ (see (3.22)), that the sign

$$
\mathbf{s}(l)=-\sigma \sigma_{1} \mathbf{s}(m) .
$$

Moreover

$$
|l| \geq|m|-\left|\sigma m+\sigma_{1} l\right| \stackrel{(3.27)}{>} \theta^{\prime} N-\mu N^{L}-\kappa N^{b} \stackrel{(3.18)}{>} \theta N .
$$

This shows that the restriction $|l|>\theta N$ in the sum (3.24) is automatically met. Then

$$
\begin{aligned}
\Pi_{N, \mu^{\prime}}^{L} \sum_{|l|>\theta N} f_{m, l}^{\sigma, \sigma_{1}} g_{l, n}^{-\sigma_{1}, \sigma^{\prime}} \stackrel{\stackrel{(3.26)}{=}}{=} \Pi_{N, \mu^{\prime}}^{L} \sum_{l \in \mathbb{Z}} f^{\sigma, \sigma_{1}}\left(\mathbf{s}(m), \sigma m+\sigma_{1} l\right) g^{-\sigma_{1}, \sigma^{\prime}}\left(\mathbf{s}(l),-\sigma_{1} l+\sigma^{\prime} n\right) \\
=\Pi_{N, \mu^{\prime}}^{L} \sum_{j \in \mathbb{Z}} f^{\sigma, \sigma_{1}}(\mathbf{s}(m), j) g^{-\sigma_{1}, \sigma^{\prime}}\left(\mathbf{s}(l), \sigma m+\sigma^{\prime} n-j\right) \\
\stackrel{(3.28)}{=} \Pi_{N, \mu^{\prime}}^{L} \sum_{j \in \mathbb{Z}} f^{\sigma, \sigma_{1}}(\mathbf{s}(m), j) g^{-\sigma_{1}, \sigma^{\prime}}\left(-\sigma \sigma_{1} \mathbf{s}(m), \sigma m+\sigma^{\prime} n-j\right)
\end{aligned}
$$

depends only on $\mathbf{s}(m)$ and $\sigma m+\sigma^{\prime} n$, i.e., (3.23). 


\subsection{Quasi-Töplitz functions}

Given $f \in \mathcal{H}_{s, r}$ and $\tilde{f} \in \mathcal{T}_{s, r}(N, \theta, \mu)$ we set

$$
\hat{f}:=N\left(\Pi_{N, \theta, \mu} f-\tilde{f}\right) .
$$

All the functions $f \in \mathcal{H}_{s, r}$ below possibly depend on parameters $\xi \in \mathcal{O}$, see (2.87). For simplicity we shall often omit this dependence and denote \|\|$_{s, r, \mathcal{O}}=\|\|_{s, r}$.

Definition 3.4 (Quasi-Töplitz). - A function $f \in \mathcal{H}_{s, r}^{\text {null }}$ is called $\left(N_{0}, \theta, \mu\right)$-quasiTöplitz if the quasi-Töplitz semi-norm

$$
\|f\|_{s, r}^{T}:=\|f\|_{s, r, N_{0}, \theta, \mu}^{T}:=\sup _{N \geq N_{0}}\left[\inf _{\tilde{f} \in \mathcal{T}_{s, r}(N, \theta, \mu)}\left(\max \left\{\left\|X_{f}\right\|_{s, r},\left\|X_{\tilde{f}}\right\|_{s, r},\left\|X_{\hat{f}}\right\|_{s, r}\right\}\right)\right]
$$

is finite. We define

$$
\mathcal{Q}_{s, r}^{T}:=\mathcal{Q}_{s, r}^{T}\left(N_{0}, \theta, \mu\right):=\left\{f \in \mathcal{H}_{s, r}^{\text {null }}:\|f\|_{s, r, N_{0}, \theta, \mu}^{T}<\infty\right\} .
$$

In other words, a function $f$ is $\left(N_{0}, \theta, \mu\right)$-quasi-Töplitz with semi-norm $\|f\|_{s, r}^{T}$ if, for all $N \geq N_{0}, \forall \varepsilon>0$, there is $\tilde{f} \in \mathcal{T}_{s, r}(N, \theta, \mu)$ such that

$$
\Pi_{N, \theta, \mu} f=\tilde{f}+N^{-1} \hat{f} \quad \text { and } \quad\left\|X_{f}\right\|_{s, r},\left\|X_{\tilde{f}}\right\|_{s, r},\left\|X_{\hat{f}}\right\|_{s, r} \leq\|f\|_{s, r}^{T}+\varepsilon .
$$

We call $\tilde{f} \in \mathcal{T}_{s, r}(N, \theta, \mu)$ a "Töplitz approximation" of $f$ and $\hat{f}$ the "Töplitz-defect". Note that, by Definition 3.3 and (3.29)

$$
\Pi_{N, \theta, \mu} \tilde{f}=\tilde{f}, \quad \Pi_{N, \theta, \mu} \hat{f}=\hat{f} .
$$

By the Definition (3.30) we get

$$
\left\|X_{f}\right\|_{s, r} \leq\|f\|_{s, r}^{T}
$$

and we complete (2.89) noting that

$$
\text { quasi-Töplitz } \Longrightarrow \text { M-regular } \Longrightarrow \text { regular } \Longleftarrow \lambda \text {-regular . }
$$

Clearly, if $f$ is $\left(N_{0}, \theta, \mu\right)$-Töplitz then $f$ is $\left(N_{0}, \theta, \mu\right)$-quasi-Töplitz and

$$
\|f\|_{s, r, N_{0}, \theta, \mu}^{T}=\left\|X_{f}\right\|_{s, r} .
$$

Then we have the following inclusions

$$
\mathcal{T}_{s, r} \subset \mathcal{Q}_{s, r}^{T}, \mathcal{B}_{s, r} \subset \mathcal{H}_{s, r}^{\text {null }} \subset \mathcal{H}_{s, r} .
$$

Note that neither $\mathcal{B}_{s, r} \subseteq \mathcal{Q}_{s, r}^{T}$ nor $\mathcal{B}_{s, r} \supseteq \mathcal{Q}_{s, r}^{T}$.

Lemma 3.3. - For parameters $N_{1} \geq N_{0}, \mu_{1} \leq \mu, \theta_{1} \geq \theta, r_{1} \leq r, s_{1} \leq s$, we have

$$
\mathcal{Q}_{s, r}^{T}\left(N_{0}, \theta, \mu\right) \subset \mathcal{Q}_{s_{1}, r_{1}}^{T}\left(N_{1}, \theta_{1}, \mu_{1}\right)
$$

and

$$
\|f\|_{s_{1}, r_{1}, N_{1}, \theta_{1}, \mu_{1}}^{T} \leq \max \left\{s / s_{1},\left(r / r_{1}\right)^{2}\right\}\|f\|_{s, r, N_{0}, \theta, \mu}^{T} .
$$


Proof. - By (3.31), for all $N \geq N_{1} \geq N_{0}$ (since $\theta_{1} \geq \theta, \mu_{1} \leq \mu$ )

$$
\Pi_{N, \theta_{1}, \mu_{1}} f=\Pi_{N, \theta_{1}, \mu_{1}} \Pi_{N, \theta, \mu} f=\Pi_{N, \theta_{1}, \mu_{1}} \tilde{f}+N^{-1} \Pi_{N, \theta_{1}, \mu_{1}} \hat{f} .
$$

The function $\Pi_{N, \theta_{1}, \mu_{1}} \tilde{f} \in \mathcal{T}_{s_{1}, r_{1}}\left(N, \theta_{1}, \mu_{1}\right)$ and

Hence, $\forall N \geq N_{1}$,

$$
\begin{gathered}
\left\|X_{\Pi_{N, \theta_{1}, \mu_{1}} \tilde{f}}\right\|_{s_{1}, r_{1}} \stackrel{(2.80)}{\leq}\left\|X_{\tilde{f}}\right\|_{s_{1}, r_{1}} \stackrel{(3.31)}{\leq}\|f\|_{s_{1}, r_{1}}^{T}+\varepsilon, \\
\left\|X_{\Pi_{N, \theta_{1}, \mu_{1}} \hat{f}}\right\|_{s_{1}, r_{1}} \stackrel{(2.80)}{\leq}\left\|X_{\hat{f}}\right\|_{s_{1}, r_{1}} \stackrel{(3.31)}{\leq}\|f\|_{s_{1}, r_{1}}^{T}+\varepsilon .
\end{gathered}
$$

$$
\inf _{\tilde{f} \in \mathcal{T}_{s_{1}, r_{1}}\left(N, \theta_{1}, \mu_{1}\right)}\left(\max \left\{\left\|X_{f}\right\|_{s_{1}, r_{1}},\left\|X_{\tilde{f}}\right\|_{s_{1}, r_{1}},\left\|X_{\hat{f}}\right\|_{s_{1}, r_{1}}\right\}\right) \leq\|f\|_{s_{1}, r_{1}}^{T}+\varepsilon,
$$

applying (2.3) we have (3.35), because $\varepsilon>0$ is arbitrary.

For $f \in \mathcal{H}_{s, r}$ we define its homogeneous component of degree $l \in \mathbb{N}$,

$$
f^{(l)}:=\Pi^{(l)} f:=\sum_{k \in \mathbb{Z}^{n}, 2|i|+|\alpha|+|\beta|=l} f_{k, i, \alpha, \beta} e^{\mathrm{i} k \cdot x} y^{i} z^{\alpha} \bar{z}^{\beta},
$$

and the projections

$$
f_{K}:=\Pi_{|k| \leq K} f:=\sum_{|k| \leq K, i, \alpha, \beta} f_{k, i, \alpha, \beta} e^{\mathrm{i} k \cdot x} y^{i} z^{\alpha} \bar{z}^{\beta}, \quad \Pi_{>K} f:=f-\Pi_{|k| \leq K} f .
$$

We also set

$$
f_{K}^{\leq 2}:=\Pi_{|k| \leq K} f^{\leq 2}, \quad f^{\leq 2}:=f^{(0)}+f^{(1)}+f^{(2)} .
$$

The above projectors $\Pi^{(l)}, \Pi_{|k| \leq K}, \Pi_{>K}$ have the form $\Pi_{I}$, see (2.13), for suitable subsets $I \subset \mathbb{I}$.

Lemma 3.4 (Projections). - Let $f \in \mathcal{Q}_{s, r}^{T}\left(N_{0}, \theta, \mu\right)$. Then, for all $l \in \mathbb{N}, K \in \mathbb{N}$,

$$
\begin{gathered}
\left\|\Pi^{(l)} f\right\|_{s, r, N_{0}, \theta, \mu}^{T} \leq\|f\|_{s, r, N_{0}, \theta, \mu}^{T} \\
\|f\|_{s, r, N_{0}, \theta, \mu},\left\|f-f_{K}^{\leq 2}\right\|_{s, r, N_{0}, \theta, \mu}^{T} \leq\|f\|_{s, r, N_{0}, \theta, \mu}^{T} \\
\left\|\Pi_{|k| \leq K} f\right\|_{s, r, N_{0}, \theta, \mu}^{T} \leq\|f\|_{s, r, N_{0}, \theta, \mu}^{T} \\
\left\|\Pi_{k=0} \Pi_{|\alpha|=|\beta|=1} \Pi^{(2)} f\right\|_{r, N_{0}, \theta, \mu}^{T} \leq\left\|\Pi^{(2)} f\right\|_{s, r, N_{0}, \theta, \mu}^{T}
\end{gathered}
$$

and, $\forall 0<s^{\prime}<s$,

$$
\left\|\Pi_{>K} f\right\|_{s^{\prime}, r, N_{0}, \theta, \mu}^{T} \leq e^{-K\left(s-s^{\prime}\right)} \frac{s}{s^{\prime}}\|f\|_{s, r, N_{0}, \theta, \mu}^{T} .
$$

Proof. - We first note that by (2.15) (recall also Remark 3.1) we have

$$
\Pi^{(l)} \Pi_{N, \theta, \mu} g=\Pi_{N, \theta, \mu} \Pi^{(l)} g, \quad \forall g \in \mathcal{H}_{s, r} .
$$

Then, applying $\Pi^{(l)}$ in (3.31), we deduce that, $\forall N \geq N_{0}, \forall \varepsilon>0$, there is $\tilde{f} \in \mathcal{T}_{s, r}(N, \theta, \mu)$ such that

$$
\Pi^{(l)} \Pi_{N, \theta, \mu} f=\Pi_{N, \theta, \mu} \Pi^{(l)} f=\Pi^{(l)} \tilde{f}+N^{-1} \Pi^{(l)} \hat{f}
$$

and, by (2.80), (3.31),

$$
\left\|X_{\Pi^{(l)} f}\right\|_{s, r},\left\|X_{\Pi^{(l)} \tilde{f}}\right\|_{s, r},\left\|X_{\Pi^{(l)} \hat{f}}\right\|_{s, r} \leq\|f\|_{s, r}^{T}+\varepsilon .
$$


We claim that $\Pi^{(l)} \tilde{f} \in \mathcal{T}_{s, r}(N, \theta, \mu), \forall l \geq 0$. Hence (3.45)-(3.46) imply $\Pi^{(l)} f \in \mathcal{Q}_{s, r}^{T}\left(N_{0}, \theta, \mu\right)$ and

$$
\left\|\Pi^{(l)} f\right\|_{s, r}^{T} \leq\|f\|_{s, r}^{T}+\varepsilon,
$$

i.e., (3.39). Let us prove our claim. For $l=0,1$ the projection $\Pi^{(l)} \tilde{f}=0$ because $\tilde{f} \in \mathcal{T}_{s, r}(N, \theta, \mu)$ is bilinear. For $l \geq 2$, write $\tilde{f}$ in the form (3.11) with coefficients $\tilde{f}_{m, n}^{\sigma, \sigma^{\prime}}$ satisfying (3.15). Then also $g:=\Pi^{(l)} \tilde{f}$ has the form (3.11) with coefficients

$$
g_{m, n}^{\sigma, \sigma^{\prime}}=\Pi^{(l-2)} \tilde{f}_{m, n}^{\sigma, \sigma^{\prime}}
$$

which satisfy (3.15) noting that $\Pi^{(l)} \mathcal{L}_{s, r}(N, \mu, h) \subset \mathcal{L}_{s, r}(N, \mu, h)$. Hence $g \in \mathcal{T}_{s, r}(N, \theta, \mu)$, $\forall l \geq 0$, proving the claim. The proof of (3.40), (3.41), (3.42), and (3.43) are similar (use also (2.57)).

Lemma 3.5. - Assume that, $\forall N \geq N_{*}$, we have the decomposition

$$
G=G_{N}^{\prime}+G_{N}^{\prime \prime} \quad \text { with } \quad\left\|G_{N}^{\prime}\right\|_{s, r, N, \theta, \mu}^{T} \leq K_{1}, \quad N\left\|X_{\Pi_{N, \theta, \mu} G_{N}^{\prime \prime}}\right\|_{s, r} \leq K_{2} .
$$

Then $\|G\|_{s, r, N_{*}, \theta, \mu}^{T} \leq \max \left\{\left\|X_{G}\right\|_{s, r}, K_{1}+K_{2}\right\}$.

Proof. - By assumption, $\forall N \geq N_{*}$, we have $\left\|G_{N}^{\prime}\right\|_{s, r, N, \theta, \mu}^{T} \leq K_{1}$. Then, $\forall \varepsilon>0$, there exist $\tilde{G}_{N}^{\prime} \in \mathcal{T}_{s, r}(N, \theta, \mu), \hat{G}_{N}^{\prime}$, such that

$$
\Pi_{N, \theta, \mu} G_{N}^{\prime}=\tilde{G}_{N}^{\prime}+N^{-1} \hat{G}_{N}^{\prime} \quad \text { and } \quad\left\|X_{\tilde{G}_{N}^{\prime}}\right\|_{s, r},\left\|X_{\hat{G}_{N}^{\prime}}\right\|_{s, r} \leq K_{1}+\varepsilon .
$$

Therefore, $\forall N \geq N_{*}$,

$$
\Pi_{N, \theta, \mu} G=\tilde{G}_{N}+N^{-1} \hat{G}_{N}, \quad \tilde{G}_{N}:=\tilde{G}_{N}^{\prime}, \quad \hat{G}_{N}:=\hat{G}_{N}^{\prime}+N \Pi_{N, \theta, \mu} G_{N}^{\prime \prime}
$$

where $\tilde{G}_{N} \in \mathcal{T}_{s, r}(N, \theta, \mu)$ and

$$
\begin{gathered}
\left\|X_{\tilde{G}_{N}}\right\|_{s, r}=\left\|X_{\tilde{G}_{N}^{\prime}}\right\|_{s, r} \stackrel{(3.48)}{\leq} K_{1}+\varepsilon, \\
\left\|X_{\hat{G}_{N}}\right\|_{s, r} \leq\left\|X_{\hat{G}_{N}^{\prime}}\right\|_{s, r}+N\left\|X_{\Pi_{N, \theta, \mu} G_{N}^{\prime \prime}}\right\|_{s, r} \stackrel{(3.48),(3.47)}{\leq} K_{1}+\varepsilon+K_{2} .
\end{gathered}
$$

Then $G \in \mathcal{Q}_{s, r, N_{*}, \theta, \mu}^{T}$ and

$$
\begin{gathered}
\|G\|_{s, r, N_{*}, \theta, \mu}^{T} \leq \sup _{N \geq N_{*}} \max \left\{\left\|X_{G}\right\|_{s, r},\left\|X_{\tilde{G}_{N}}\right\|_{s, r},\left\|X_{\hat{G}_{N}}\right\|_{s, r}\right\} \\
\stackrel{(3.49),(3.50)}{\leq} \max \left\{\left\|X_{G}\right\|_{s, r}, K_{1}+K_{2}+\varepsilon\right\} .
\end{gathered}
$$

Since $\varepsilon>0$ is arbitrary the lemma follows.

The Poisson bracket of two quasi-Töplitz functions is quasi-Töplitz.

Proposition 3.1 (Poisson bracket). - Assume that $f^{(1)}, f^{(2)} \in \mathcal{Q}_{s, r}^{T}\left(N_{0}, \theta, \mu\right)$ and $N_{1} \geq N_{0}, \mu_{1} \leq \mu, \theta_{1} \geq \theta, s / 2 \leq s_{1}<s, r / 2 \leq r_{1}<r$ satisfy

(3.51) $\kappa N_{1}^{b-L}<\mu-\mu_{1}, \mu N_{1}^{L-1}+\kappa N_{1}^{b-1}<\theta_{1}-\theta, 2 N_{1} e^{-N_{1}^{b} \frac{s-s_{1}}{2}}<1, b\left(s-s_{1}\right) N_{1}^{b}>2$.

Then

and

$$
\left\{f^{(1)}, f^{(2)}\right\} \in \mathcal{Q}_{s_{1}, r_{1}}^{T}\left(N_{1}, \theta_{1}, \mu_{1}\right)
$$


where $C(n) \geq 1$ and

$$
\delta:=\min \left\{1-\frac{s_{1}}{s}, 1-\frac{r_{1}}{r}\right\} .
$$

The proof is based on the following splitting lemma for the Poisson brackets.

Lemma 3.6 (Splitting lemma). - Let $f^{(1)}, f^{(2)} \in \mathcal{Q}_{s, r}^{T}\left(N_{0}, \theta, \mu\right)$ and (3.51) hold. Then, for all $N \geq N_{1}$,

$$
\begin{aligned}
\Pi_{N, \theta_{1}, \mu_{1}}\left\{f^{(1)}, f^{(2)}\right\}= & \Pi_{N, \theta_{1}, \mu_{1}}\left(\left\{\Pi_{N, \theta, \mu} f^{(1)}, \Pi_{N, \theta, \mu} f^{(2)}\right\}^{H}\right. \\
& +\left\{\Pi_{N, \theta, \mu} f^{(1)}, \Pi_{N, 2 \mu}^{L} f^{(2)}\right\}^{L}+\left\{\Pi_{N, 2 \mu}^{L} f^{(1)}, \Pi_{N, \theta, \mu} f^{(2)}\right\}^{L} \\
& +\left\{\Pi_{N, \theta, \mu} f^{(1)}, \Pi_{N, \mu}^{L} f^{(2)}\right\}^{x, y}+\left\{\Pi_{N, \mu}^{L} f^{(1)}, \Pi_{N, \theta, \mu} f^{(2)}\right\}^{x, y} \\
& \left.+\left\{\Pi_{|k| \geq N^{b}} f^{(1)}, f^{(2)}\right\}+\left\{\Pi_{|k|<N^{b}} f^{(1)}, \Pi_{|k| \geq N^{b}} f^{(2)}\right\}\right) .
\end{aligned}
$$

Proof. - We have

$$
\begin{aligned}
\left\{f^{(1)}, f^{(2)}\right\}= & \left\{\Pi_{|k|<N^{b}} f^{(1)}, \Pi_{|k|<N^{b}} f^{(2)}\right\} \\
& +\left\{\Pi_{|k| \geq N^{b}} f^{(1)}, f^{(2)}\right\}+\left\{\Pi_{|k|<N^{b}} f^{(1)}, \Pi_{|k| \geq N^{b}} f^{(2)}\right\} .
\end{aligned}
$$

The last two terms correspond to the last line in (3.54). We now study the first term in the right hand side of (3.55). We replace each $f^{(i)}, i=1,2$, with single monomials (with zero momentum) and we analyze under which conditions the projection

$$
\Pi_{N, \theta_{1}, \mu_{1}}\left\{e^{\mathrm{i} k^{(1)} \cdot x} y^{i^{(1)}} z^{\alpha^{(1)}} \bar{z}^{\beta^{(1)}}, e^{\mathrm{i} k^{(2)} \cdot x} y^{i^{(2)}} z^{\alpha^{(2)}} \bar{z}^{\beta^{(2)}}\right\}, \quad\left|k^{(1)}\right|,\left|k^{(2)}\right|<N^{b},
$$

is not zero. By direct inspection, recalling the Definition 3.2 of $\Pi_{N, \theta_{1}, \mu_{1}}$ and the expression (2.81) of the Poisson brackets $\{\}=,\{,\}^{x, y}+\{,\}^{z, \bar{z}}$, one of the following situations (apart from a trivial permutation of the indices 1,2$)$ must hold:

1. one has $z^{\alpha^{(1)}} \bar{z}^{\beta^{(1)}}=z^{\tilde{\alpha}^{(1)}} \bar{z}^{\tilde{\beta}^{(1)}} z_{m}^{\sigma} z_{j}^{\sigma_{1}}$ and $z^{\alpha^{(2)}} \bar{z}^{\beta^{(2)}}=z^{\tilde{\alpha}^{(2)}} \bar{z}^{\tilde{\beta}^{(2)}} z_{n}^{\sigma^{\prime}} z_{j}^{-\sigma_{1}}$ where $|m|,|n| \geq \theta_{1} N, \sigma, \sigma_{1}, \sigma^{\prime}= \pm$, and $z^{\tilde{\alpha}^{(1)}} \bar{z}^{\tilde{\beta}^{(1)}} z^{\tilde{\alpha}^{(2)}} \bar{z}^{\tilde{\beta}^{(2)}}$ is of $\left(N, \mu_{1}\right)$-low momentum. We consider the Poisson bracket $\{,\}^{z, \bar{z}}$ (in the variables $\left(z_{j}^{+}, z_{j}^{-}\right)$) of the monomials.

2. one has $z^{\alpha^{(1)}} \bar{z}^{\beta^{(1)}}=z^{\tilde{\alpha}^{(1)}} \bar{z}^{\tilde{\beta}^{(1)}} z_{m}^{\sigma} z_{n}^{\sigma^{\prime}} z_{j}^{\sigma_{1}}$ and $z^{\alpha^{(2)}} \bar{z}^{\beta^{(2)}}=z^{\tilde{\alpha}^{(2)}} \bar{z}^{\tilde{\beta}^{(2)}} z_{j}^{-\sigma_{1}}$ where $|m|,|n| \geq \theta_{1} N$ and $z^{\tilde{\alpha}^{(1)}} \bar{z}^{\tilde{\beta}^{(1)}} z^{\tilde{\alpha}^{(2)}} \bar{z}^{\tilde{\beta}^{(2)}}$ is of $\left(N, \mu_{1}\right)$-low momentum. We consider the Poisson bracket $\{,\}^{z, \bar{z}}$.

3. one has $z^{\alpha^{(1)}} \bar{z}^{\beta^{(1)}}=z^{\tilde{\alpha}^{(1)}} \bar{z}^{\tilde{\beta}^{(1)}} z_{m}^{\sigma} z_{n}^{\sigma^{\prime}}$ and $z^{\alpha^{(2)}} \bar{z}^{\beta^{(2)}}=z^{\tilde{\alpha}^{(2)}} \bar{z}^{\tilde{\beta}^{(2)}}$, where $|m|,|n| \geq \theta_{1} N$ and $z^{\tilde{\alpha}^{(1)}} \bar{z}^{\tilde{\beta}^{(1)}} z^{\tilde{\alpha}^{(2)}} \bar{z}^{\tilde{\beta}^{(2)}}$ is of $\left(N, \mu_{1}\right)$-low momentum. We consider the Poisson bracket $\{,\}^{x, y}$, i.e., in the variables $(x, y)$.

Note that when we consider the $\{,\}^{x, y}$ Poisson bracket, the case

$$
z^{\alpha^{(1)}} \bar{z}^{\beta^{(1)}}=z^{\tilde{\alpha}^{(1)}} \bar{z}^{\tilde{\beta}^{(1)}} z_{m}^{\sigma} \quad \text { and } \quad z^{\alpha^{(2)}} \bar{z}^{\beta^{(2)}}=z^{\tilde{\alpha}^{(2)}} \bar{z}^{\tilde{\beta}^{(2)}} z_{n}^{\sigma^{\prime}},|m|,|n| \geq \theta_{1} N,
$$

and $z^{\tilde{\alpha}^{(1)}} \bar{z}^{\tilde{\beta}^{(1)}} z^{\tilde{\alpha}^{(2)}} \bar{z}^{\tilde{\beta}^{(2)}}$ is of $\left(N, \mu_{1}\right)$-low momentum, does not appear. Indeed, the momentum conservation $-\sigma m=\pi\left(\tilde{\alpha}^{(1)}, \tilde{\beta}^{(1)}, k^{(1)}\right),(2.86)$ and $\left|k^{(1)}\right|<N^{b}$, give

$$
\theta_{1} N<|m| \leq \sum_{l \in \mathbb{Z} \backslash \mathcal{I}}|l|\left(\left|\tilde{\alpha}_{l}^{(1)}\right|+\left|\tilde{\beta}_{l}^{(1)}\right|\right)+\kappa N^{b} \leq \mu_{1} N^{L}+\kappa N^{b},
$$


which contradicts (3.1).

CASE 1. The momentum conservation of each monomial gives

$$
\sigma_{1} j=-\sigma m-\pi\left(\tilde{\alpha}^{(1)}, \tilde{\beta}^{(1)}, k^{(1)}\right)=\sigma^{\prime} n+\pi\left(\tilde{\alpha}^{(2)}, \tilde{\beta}^{(2)}, k^{(2)}\right) .
$$

Since $z^{\tilde{\alpha}^{(1)}} \bar{z}^{\tilde{\beta}^{(1)}} z^{\tilde{\alpha}^{(2)}} \bar{z}^{\tilde{\beta}^{(2)}}$ is of $\left(N, \mu_{1}\right)$-low momentum (Definition 3.1),

$$
\sum_{l \in \mathbb{Z} \backslash \mathcal{I}}|l|\left(\tilde{\alpha}_{l}^{(1)}+\tilde{\beta}_{l}^{(1)}+\tilde{\alpha}_{l}^{(2)}+\tilde{\beta}_{l}^{(2)}\right) \leq \mu_{1} N^{L} \Longrightarrow \sum_{l \in \mathbb{Z} \backslash \mathcal{I}}|l|\left(\tilde{\alpha}_{l}^{(i)}+\tilde{\beta}_{l}^{(i)}\right) \leq \mu_{1} N^{L}, i=1,2,
$$

which implies, by (3.56), (2.86), $\left|k^{(1)}\right|<N^{b},|j| \geq \theta_{1} N-\mu_{1} N^{L}-\kappa N^{b}>\theta N$ by (3.51). Hence $|m|,|n|,|j|>\theta N$. Then $e^{\mathrm{i} k^{(h)} \cdot x} y^{i^{(h)}} z^{\alpha^{(h)}} \bar{z}^{\beta^{(h)}}, h=1,2$, are $(N, \theta, \mu)$-bilinear. Moreover the $\left(z_{j}, \bar{z}_{j}\right)$ are high momentum variables, namely $\{,\}^{z, \bar{z}}=\{,\}^{H}$, see (3.4). As $m, n$ run over all $\mathbb{Z} \backslash \mathcal{I}$ with $|m|,|n| \geq \theta_{1} N$, we obtain the first term in Formula (3.54).

CASE 2. The momentum conservation of the second monomial reads

$$
-\sigma_{1} j=-\pi\left(\tilde{\alpha}^{(2)}, \tilde{\beta}^{(2)}, k^{(2)}\right) .
$$

Then, using also (2.86), $\left|k^{(2)}\right|<N^{b}$, that $z^{\tilde{\alpha}^{(1)}} \bar{z}^{\tilde{\beta}^{(1)}} z^{\tilde{\alpha}^{(2)}} \bar{z}^{\tilde{\beta}^{(2)}}$ is of $\left(N, \mu_{1}\right)$-low momentum,

$$
\begin{aligned}
|j|+\sum_{l \in \mathbb{Z} \backslash \mathcal{I}}|l|\left(\tilde{\alpha}_{l}^{(1)}+\tilde{\beta}_{l}^{(1)}\right) \stackrel{(3.57)}{=}\left|\pi\left(\tilde{\alpha}^{(2)}, \tilde{\beta}^{(2)}, k^{(2)}\right)\right|+\sum_{l \in \mathbb{Z} \backslash \mathcal{I}}|l|\left(\tilde{\alpha}_{l}^{(1)}+\tilde{\beta}_{l}^{(1)}\right) \\
\quad \leq \sum_{l \in \mathbb{Z} \backslash \mathcal{I}}|l|\left(\tilde{\alpha}_{l}^{(1)}+\tilde{\beta}_{l}^{(1)}+\tilde{\alpha}_{l}^{(2)}+\tilde{\beta}_{l}^{(2)}\right)+\kappa N^{b} \leq \mu_{1} N^{L}+\kappa N^{b} \stackrel{(3.51)}{<} \mu N^{L} .
\end{aligned}
$$

Then $z^{\tilde{\alpha}^{(1)}} \bar{z}^{\tilde{\beta}^{(1)}} z_{j}^{\sigma_{1}}$ is of $\left(N, \mu_{1}\right)$-low momentum and the first monomial

$$
e^{\mathrm{i} k^{(1)} \cdot x} y^{i^{(1)}} z^{\alpha^{(1)}} \bar{z}^{\beta^{(1)}}=e^{\mathrm{i} k^{(1)} \cdot x} y^{i^{(1)}} z^{\tilde{\alpha}^{(1)}} \bar{z}^{\tilde{\beta}^{(1)}} z_{j}^{\sigma_{1}} z_{m}^{\sigma} z_{n}^{\sigma^{\prime}}
$$

is $(N, \theta, \mu)$-bilinear $\left(\mu_{1} \leq \mu\right)$. The second monomial

$$
e^{\mathrm{i} k^{(2)} \cdot x} y^{i^{(2)}} z^{\alpha^{(2)}} \bar{z}^{\beta^{(2)}}=e^{\mathrm{i} k^{(2)} \cdot x} y^{i^{(2)}} z^{\tilde{\alpha}^{(2)}} \bar{z}^{\tilde{\beta}^{(2)}} z_{j}^{-\sigma_{1}}
$$

is $(N, 2 \mu)$-low-momentum because, arguing as above,

$$
\begin{aligned}
|j|+\sum_{l}|l|\left(\tilde{\alpha}_{l}^{(2)}+\tilde{\beta}_{l}^{(2)}\right) \stackrel{(3.57)}{=}\left|\pi\left(\tilde{\alpha}^{(2)}, \tilde{\beta}^{(2)}, k^{(2)}\right)\right|+\sum_{l}|l|\left(\tilde{\alpha}_{l}^{(2)}+\tilde{\beta}_{l}^{(2)}\right) \\
\leq 2 \mu_{1} N^{L}+\kappa N^{b} \stackrel{(3.51)}{<} 2 \mu N^{L} .
\end{aligned}
$$

The $\left(z_{j}, \bar{z}_{j}\right)$ are low momentum variables, namely $\{,\}^{z, \bar{z}}=\{,\}^{L}$, and we obtain the second and third contribution in Formula (3.54).

CASE 3. We have, for $i=1,2$, that

$$
\sum_{l}|l|\left(\tilde{\alpha}_{l}^{(i)}+\tilde{\beta}_{l}^{(i)}\right) \leq \sum_{l}|l|\left(\tilde{\alpha}_{l}^{(1)}+\tilde{\beta}_{l}^{(1)}+\tilde{\alpha}_{l}^{(2)}+\tilde{\beta}_{l}^{(2)}\right) \leq \mu_{1} N^{L} \leq \mu N^{L}
$$

Then $e^{\mathrm{i} k^{(1)} \cdot x} y^{i^{(1)}} z^{\alpha^{(1)}} \bar{z}^{\beta^{(1)}}$ is $(N, \theta, \mu)$-bilinear and $e^{\mathrm{i} k^{(2)} \cdot x} y^{i^{(2)}} z^{\alpha^{(2)}} \bar{z}^{\beta^{(2)}}$ is $(N, \mu)$-lowmomentum. We obtain the fourth and fifth contribution in Formula (3.54). 
Proof of Proposition 3.1. - Since $f^{(i)} \in \mathcal{Q}_{s, r}^{T}\left(N_{0}, \theta, \mu\right), i=1,2$, for all $N \geq N_{1} \geq N_{0}$ there exist $\tilde{f}^{(i)} \in \mathcal{T}_{s, r}(N, \theta, \mu)$ and $\hat{f}^{(i)}$ such that (see (3.31))

$$
\Pi_{N, \theta, \mu} f^{(i)}=\tilde{f}^{(i)}+N^{-1} \hat{f}^{(i)}, \quad i=1,2,
$$

and

$$
\left\|X_{f^{(i)}}\right\|_{s, r},\left\|X_{\tilde{f}^{(i)}}\right\|_{s, r},\left\|X_{\hat{f}^{(i)}}\right\|_{s, r} \leq 2\left\|f^{(i)}\right\|_{s, r}^{T} .
$$

In order to show that $\left\{f^{(1)}, f^{(2)}\right\} \in \mathcal{Q}_{s_{1}, r_{1}}^{T}\left(N_{1}, \theta_{1}, \mu_{1}\right)$ and prove (3.52) we have to provide a decomposition

$$
\Pi_{N, \theta_{1}, \mu_{1}}\left\{f^{(1)}, f^{(2)}\right\}=\tilde{f}^{(1,2)}+N^{-1} \hat{f}^{(1,2)}, \quad \forall N \geq N_{1},
$$

so that $\tilde{f}^{(1,2)} \in \mathcal{T}_{s_{1}, r_{1}}\left(N, \theta_{1}, \mu_{1}\right)$ and

$$
\left\|X_{\left\{f^{(1)}, f^{(2)}\right\}}\right\|_{s_{1}, r_{1}},\left\|X_{\tilde{f}^{(1,2)}}\right\|_{s_{1}, r_{1}},\left\|X_{\hat{f}^{(1,2)}}\right\|_{s_{1}, r_{1}}<C(n) \delta^{-1}\left\|f^{(1)}\right\|_{s, r}^{T}\left\|f^{(2)}\right\|_{s, r}^{T}
$$

(for brevity we omit the indices $N_{1}, \theta_{1}, \mu_{1}, N_{0}, \theta, \mu$ ). By (2.92) we have ( $\delta$ is defined in (3.53))

$$
\left\|X_{\left\{f^{(1)}, f^{(2)}\right\}}\right\|_{s_{1}, r_{1}} \leq 2^{2 n+3} \delta^{-1}\left\|X_{f^{(1)}}\right\|_{s, r}\left\|X_{f^{(2)}}\right\|_{s, r} .
$$

Considering (3.58) and (3.54), we define the candidate Töplitz approximation

$$
\begin{aligned}
\tilde{f}^{(1,2)}:=\Pi_{N, \theta_{1}, \mu_{1}}\left(\left\{\tilde{f}^{(1)}, \tilde{f}^{(2)}\right\}^{H}+\left\{\tilde{f}^{(1)}, \Pi_{N, 2 \mu}^{L} f^{(2)}\right\}^{L}+\left\{\Pi_{N, 2 \mu}^{L} f^{(1)}, \tilde{f}^{(2)}\right\}^{L}\right. \\
+\left\{\tilde{f}^{(1)}, \Pi_{N, \mu}^{L} f^{(2)}\right\}^{x, y}+\left\{\Pi_{N, \mu}^{L} f^{(1)}, \tilde{f}^{(2)}\right\}^{x, y}
\end{aligned}
$$

and Töplitz-defect

$$
\hat{f}^{(1,2)}:=N\left(\Pi_{N, \theta_{1}, \mu_{1}}\left\{f^{(1)}, f^{(2)}\right\}-\tilde{f}^{(1,2)}\right) .
$$

Lemma 3.2 and (3.51) imply that $\tilde{f}^{(1,2)} \in \mathcal{T}_{s_{1}, r_{1}}\left(N, \theta_{1}, \mu_{1}\right)$. The estimate (3.60) for $\tilde{f}^{(1,2)}$ follows by (3.61), (2.92), (2.80), (3.59). Next

$$
\begin{aligned}
\hat{f}^{(1,2)}= & \Pi_{N, \theta_{1}, \mu_{1}}\left(\left\{\tilde{f}^{(1)}, \hat{f}^{(2)}\right\}^{H}+\left\{\hat{f}^{(1)}, \tilde{f}^{(2)}\right\}^{H}+N^{-1}\left\{\hat{f}^{(1)}, \hat{f}^{(2)}\right\}^{H}\right. \\
& +\left\{\hat{f}^{(1)}, \Pi_{N, 2 \mu}^{L} f^{(2)}\right\}^{L}+\left\{\Pi_{N, 2 \mu}^{L} f^{(1)}, \hat{f}^{(2)}\right\}^{L} \\
& +\left\{\hat{f}^{(1)}, \Pi_{N, \mu}^{L} f^{(2)}\right\}^{x, y}+\left\{\Pi_{N, \mu}^{L} f^{(1)}, \hat{f}^{(2)}\right\}^{x, y} \\
& \left.+N\left\{\Pi_{|k| \geq N^{b}} f^{(1)}, f^{(2)}\right\}+N\left\{\Pi_{|k|<N^{b}} f^{(1)}, \Pi_{|k| \geq N^{b}} f^{(2)}\right\}\right)
\end{aligned}
$$

and the bound (3.60) follows again by (2.92), (2.80), (3.59), (2.57), (3.51). Let us consider only the term $N\left\{\Pi_{|k| \geq N^{b}} f^{(1)}, f^{(2)}\right\}=: g$, the last one being analogous. We first use Lemma 2.16 with $r^{\prime} \rightsquigarrow r_{1}, r \rightsquigarrow r, s^{\prime} \rightsquigarrow s_{1}$ and $s \rightsquigarrow s_{1}+\sigma / 2$, where $\sigma:=s-s_{1}$. Since $\left(1-\frac{s_{1}}{s_{1}+\sigma / 2}\right)^{-1} \leq$ $2\left(1-\frac{s_{1}}{s}\right)^{-1} \leq 2 \delta^{-1}$ with the $\delta$ in (3.53), by (2.92) we get

$$
\begin{aligned}
\left\|X_{g}\right\|_{s_{1}, r_{1}} & \leq C(n) \delta^{-1} N\left\|X_{\Pi_{|k| \geq N^{b}} f^{(1)}}\right\|_{s_{1}+\sigma / 2, r}\left\|X_{f^{(2)}}\right\|_{s, r} \\
& \stackrel{(2.57)}{\leq} C(n) \delta^{-1} N \frac{s}{s_{1}} e^{-N^{b}\left(s-s_{1}\right) / 2}\left\|X_{f^{(1)}}\right\|_{s, r}\left\|X_{f^{(2)}}\right\|_{s, r} \\
& \stackrel{(3.51)}{\leq} C(n) \delta^{-1}\left\|X_{f^{(1)}}\right\|_{s, r}\left\|X_{f^{(2)}}\right\|_{s, r},
\end{aligned}
$$

$4^{\text {e }}$ SÉRIE - TOME $46-2013$ - No 2 
for every $N \geq N_{1}$. The proof of Proposition 3.1 is complete.

The quasi-Töplitz character of a function is preserved under the flow generated by a quasiTöplitz Hamiltonian.

Proposition 3.2 (Lie transform). - Let $f, g \in \mathcal{Q}_{s, r}^{T}\left(N_{0}, \theta, \mu\right)$ and let $s / 2 \leq s^{\prime}<s$, $r / 2 \leq r^{\prime}<r$. There is $c(n)>0$ such that, if

$$
\|f\|_{s, r, N_{0}, \theta, \mu}^{T} \leq c(n) \delta
$$

with $\delta$ defined in (2.66), then the hamiltonian flow of $f$ at time $t=1, \Phi_{f}^{1}: D\left(s^{\prime}, r^{\prime}\right) \rightarrow D(s, r)$ is well defined, analytic and symplectic, and, for

$$
N_{0}^{\prime} \geq \max \left\{N_{0}, \bar{N}\right\}, \quad \bar{N}:=\exp \left(\max \left\{\frac{2}{b}, \frac{1}{L-b}, \frac{1}{1-L}, 8\right\}\right),
$$

(recall (3.2)), $\mu^{\prime}<\mu, \theta^{\prime}>\theta$, satisfying

$$
\kappa\left(N_{0}^{\prime}\right)^{b-L} \ln N_{0}^{\prime} \leq \mu-\mu^{\prime},(6+\kappa)\left(N_{0}^{\prime}\right)^{L-1} \ln N_{0}^{\prime} \leq \theta^{\prime}-\theta, 2\left(N_{0}^{\prime}\right)^{-b} \ln ^{2} N_{0}^{\prime} \leq b\left(s-s^{\prime}\right),
$$

we have $e^{\operatorname{ad}_{f}} g \in \mathcal{Q}_{s^{\prime}, r^{\prime}}^{T}\left(N_{0}^{\prime}, \theta^{\prime}, \mu^{\prime}\right)$ and

$$
\left\|e^{\operatorname{ad}_{f}} g\right\|_{s^{\prime}, r^{\prime}, N_{0}^{\prime}, \theta^{\prime}, \mu^{\prime}}^{T} \leq 2\|g\|_{s, r, N_{0}, \theta, \mu}^{T} .
$$

Moreover, for $h=0,1,2$, and coefficients $0 \leq b_{j} \leq 1 / j !, j \in \mathbb{N}$,

$$
\left\|\sum_{j \geq h} b_{j} \operatorname{ad}_{f}^{j}(g)\right\|_{s^{\prime}, r^{\prime}, N_{0}^{\prime}, \theta^{\prime}, \mu^{\prime}}^{T} \leq 2\left(C \delta^{-1}\|f\|_{s, r, N_{0}, \theta, \mu}^{T}\right)^{h}\|g\|_{s, r, N_{0}, \theta, \mu}^{T} .
$$

Note that (3.66) is (3.67) with $h=0, b_{j}:=1 / j$ !

Proof. - Let us prove (3.67). We define

$$
G^{(0)}:=g, \quad G^{(j)}:=\operatorname{ad}_{f}^{j}(g):=\operatorname{ad}_{f}\left(G^{(j-1)}\right)=\left\{f, G^{(j-1)}\right\}, j \geq 1,
$$

and we split, for $h=0,1,2$,

$$
G^{\geq h}:=\sum_{j \geq h} b_{j} G^{(j)}=\sum_{j=h}^{J-1} b_{j} G^{(j)}+\sum_{j \geq J} b_{j} G^{(j)}=: G_{<J}^{\geq h}+G_{\geq J} .
$$

As in (2.98) we deduce

$$
\left\|X_{G^{(j)}}\right\|_{s^{\prime}, r^{\prime}} \leq\left(C(n) j \delta^{-1}\right)^{j}\left\|X_{f}\right\|_{s, r}^{j}\left\|X_{g}\right\|_{s, r}, \quad \forall j \geq 0
$$

where $\delta$ is defined in (2.66). Let

$$
\eta:=C(n) e \delta^{-1}\left\|X_{f}\right\|_{s, r}<1 /(2 e)
$$

(namely take $c(n)$ small in (3.63)). By 3.69, using $j^{j} b_{j} \leq j^{j} / j !<e^{j}$, we get

$$
\left\|X_{G_{\geq J}}\right\|_{s^{\prime}, r^{\prime}} \leq \sum_{j \geq J} b_{j}\left(C(n) j \delta^{-1}\left\|X_{f}\right\|_{s, r}\right)^{j}\left\|X_{g}\right\|_{s, r} \leq 2 \eta^{J}\left\|X_{g}\right\|_{s, r} .
$$

In particular, for $J=h=0,1,2$, we get

$$
\left\|X_{G \geq h}\right\|_{s^{\prime}, r^{\prime}} \leq 2 \eta^{h}\left\|X_{g}\right\|_{s, r} .
$$

For any $N \geq N_{0}^{\prime}$ we choose

$$
J:=J(N):=\ln N
$$


and we set

$$
G_{N}^{\prime}:=G_{<J}^{\geq h}, \quad G_{N}^{\prime \prime}:=G_{\geq J}, \quad G^{\geq h}=G_{N}^{\prime}+G_{N}^{\prime \prime} .
$$

Then (3.67) follows by Lemma 3.5 (with $N_{*} \rightsquigarrow N_{0}^{\prime}, s \rightsquigarrow s^{\prime}, r \rightsquigarrow r^{\prime}, \theta \rightsquigarrow \theta^{\prime}, \mu \rightsquigarrow \mu^{\prime}$ ) and (3.72), once we show that

$$
\left\|G_{N}^{\prime}\right\|_{s^{\prime}, r^{\prime}, N, \theta^{\prime}, \mu^{\prime}}^{T} \leq \frac{3}{2} \eta^{h}\|g\|_{s, r}^{T}, \quad N\left\|X_{G_{N}^{\prime \prime}}\right\|_{s^{\prime}, r^{\prime}} \leq \frac{1}{2} \eta^{h}\|g\|_{s, r}^{T}
$$

with $h=0,1,2$ (for simplicity $\|g\|_{s, r}^{T}:=\|g\|_{s, r, N_{0}, \theta, \mu}^{T}$ ).

For all $N \geq N_{0}^{\prime} \geq e^{8}$ (recall (3.64)),

$$
\begin{aligned}
N\left\|X_{G_{\geq J}}\right\|_{s^{\prime}, r^{\prime}} & \stackrel{(3.71)}{\leq} N 2 \eta^{J}\left\|X_{g}\right\|_{s, r} \leq \eta^{h}\left(N 2 \eta^{J-h}\right)\|g\|_{s, r}^{T} \\
& \stackrel{(3.70)}{\leq} \eta^{h} 2^{-J+h+1} e^{h} N e^{-J}\|g\|_{s, r}^{T} \leq \frac{\eta^{h}}{2}\|g\|_{s, r}^{T},
\end{aligned}
$$

proving the second inequality in (3.74). Let us prove the first inequality in (3.74).

Claim: $\forall j=1, \ldots, J-1$, we have $G^{(j)} \in \mathcal{Q}_{s^{\prime}, r^{\prime}}^{T}\left(N, \theta^{\prime}, \mu^{\prime}\right)$ and

$$
\left\|G^{(j)}\right\|_{r^{\prime}, s^{\prime}, N, \theta^{\prime}, \mu^{\prime}}^{T} \leq\|g\|_{s, r}^{T}\left(C^{\prime} j \delta^{-1}\|f\|_{s, r}^{T}\right)^{j}
$$

(for simplicity $\|f\|_{s, r}^{T}:=\|f\|_{s, r, N_{0}, \theta, \mu}^{T}$ ). This claim implies (using $j^{j} b_{j}<e^{j}$ )

$\left\|\sum_{j=h}^{J-1} b_{j} G^{(j)}\right\|_{s^{\prime}, r^{\prime}, N, \theta^{\prime}, \mu^{\prime}}^{T} \stackrel{(3.76)}{\leq} \sum_{j=h}^{J-1} b_{j}\|g\|_{s, r}^{T}\left(C^{\prime} j \delta^{-1}\|f\|_{s, r}^{T}\right)^{j} \stackrel{(3.70)}{\leq}\|g\|_{s, r}^{T} \sum_{j=h}^{+\infty} \eta^{j} \leq \frac{3}{2} \eta^{h}\|g\|_{s, r}^{T}$

for $c$ small enough in (3.63). This proves the first inequality in (3.74).

Let us prove the claim. Fix $0 \leq j \leq J-1$. We define, $\forall i=0, \ldots, j$,

$$
\mu_{i}:=\mu-i \frac{\mu-\mu^{\prime}}{j}, \theta_{i}:=\theta+i \frac{\theta^{\prime}-\theta}{j}, r_{i}:=r-i \frac{r-r^{\prime}}{j}, s_{i}:=s-i \frac{s-s^{\prime}}{j},
$$

and we prove inductively that, for all $i=0, \ldots, j$,

$$
\left\|\operatorname{ad}_{f}^{i}(g)\right\|_{s_{i}, r_{i}, N, \theta_{i}, \mu_{i}}^{T} \leq\left(C^{\prime} j \delta^{-1}\|f\|_{s, r}^{T}\right)^{i}\|g\|_{s, r}^{T},
$$

which, for $i=j$, gives (3.76). For $i=0$, Formula (3.78) follows because $g \in \mathcal{Q}_{s, r}^{T}\left(N_{0}, \theta, \mu\right)$ and Lemma 3.3.

Now assume that (3.78) holds for $i$ and prove it for $i+1$. We want to apply Proposition 3.1 to the functions $f$ and $\operatorname{ad}_{f}^{i}(g)$ with $N_{1} \rightsquigarrow N, s \rightsquigarrow s_{i}, s_{1} \rightsquigarrow s_{i+1}, \theta \rightsquigarrow \theta_{i}, \theta_{1} \rightsquigarrow \theta_{i+1}$, etc. We have to verify conditions (3.51) that reads

$$
\begin{aligned}
& \kappa N^{b-L}<\mu_{i}-\mu_{i+1}, \quad \mu_{i} N^{L-1}+\kappa N^{b-1}<\theta_{i+1}-\theta_{i}, \\
& 2 N e^{-N^{b} \frac{s_{i}-s_{i+1}}{2}<1,} \quad b\left(s_{i}-s_{i+1}\right) N^{b}>2 .
\end{aligned}
$$

Since, by (3.77),

$$
\mu_{i}-\mu_{i+1}=\frac{\mu-\mu^{\prime}}{j}, \quad \theta_{i+1}-\theta_{i}=\frac{\theta-\theta^{\prime}}{j}, \quad s_{i}-s_{i+1}=\frac{s-s^{\prime}}{j}
$$

and $j<J=\ln N$ (see (3.73)), $0<b<L<1$ (recall (3.2)), $\mu^{\prime} \leq \mu \leq 6$, the above conditions (3.79)-(3.80) are implied by

$$
\begin{gathered}
\kappa N^{b-L} \ln N<\mu-\mu^{\prime}, \quad(6+\kappa) N^{L-1} \ln N<\theta^{\prime}-\theta, \\
2 N e^{-N^{b}\left(s-s^{\prime}\right) / 2 \ln N}<1, \quad b\left(s-s^{\prime}\right) N^{b}>2 \ln N .
\end{gathered}
$$


The last two conditions (3.81) are implied by $b\left(s-s^{\prime}\right) N^{b}>2 \ln ^{2} N$ and since $N \geq e^{1 / 1-b}$ (recall (3.64)). Recollecting we have to verify

$$
\kappa N^{b-L} \ln N \leq \mu-\mu^{\prime},(6+\kappa) N^{L-1} \ln N \leq \theta^{\prime}-\theta, 2 N^{-b} \ln ^{2} N \leq b\left(s-s^{\prime}\right) .
$$

Since the function $N \mapsto N^{-\gamma} \ln N$ is decreasing for $N \geq e^{1 / \gamma}$, we have that (3.82) follows by (3.64)-(3.65). Therefore Proposition 3.1 implies that $\operatorname{ad}_{f}^{i+1}(g) \in \mathcal{Q}_{s_{i+1}, r_{i+1}}^{T}\left(N, \theta_{i+1}, \mu_{i+1}\right)$ and, by (3.52), (3.35), we get

$$
\left\|\operatorname{ad}_{f}^{i+1}(g)\right\|_{s_{i+1}, r_{i+1}, N, \theta_{i+1}, \mu_{i+1}}^{T} \leq C^{\prime} \delta_{i}^{-1}\|f\|_{s, r}^{T}\left\|\operatorname{ad}_{f}^{i}(g)\right\|_{s_{i}, r_{i}, N, \theta_{i}, \mu_{i}}^{T}
$$

where

$$
\delta_{i}:=\min \left\{1-\frac{s_{i+1}}{s_{i}}, 1-\frac{r_{i+1}}{r_{i}}\right\} \geq \frac{\delta}{j}
$$

and $\delta$ is defined in (2.66). Then

$$
\begin{aligned}
\left\|\operatorname{ad}_{f}^{i+1}(g)\right\|_{s_{i+1}, r_{i+1}, N, \theta_{i+1}, \mu_{i+1}}^{T} \stackrel{(3.83),(3.84)}{\leq} C^{\prime} j \delta^{-1}\|f\|_{s, r, N_{0}, \theta, \mu}^{T}\left\|\operatorname{ad}_{f}^{i}(g)\right\|_{s_{i}, r_{i}, N, \theta_{i}, \mu_{i}}^{T} \\
\stackrel{(3.78)}{\leq}\left(C^{\prime} j \delta^{-1}\|f\|_{s, r}^{T}\right)^{i+1}\|g\|_{s, r}^{T}
\end{aligned}
$$

proving (3.78) by induction.

\section{An abstract KAM theorem}

We consider a family of integrable Hamiltonians

$$
\mathcal{N}:=\mathcal{N}(x, y, z, \bar{z} ; \xi):=e(\xi)+\omega(\xi) \cdot y+\Omega(\xi) \cdot z \bar{z}
$$

defined on $\mathbb{T}_{s}^{n} \times \mathbb{C}^{n} \times \ell_{\mathcal{I}}^{a, p} \times \ell_{\mathcal{I}}^{a, p}$, where $\mathcal{I}$ is defined in (2.84), the tangential frequencies $\omega:=\left(\omega_{1}, \ldots, \omega_{n}\right)$ and the normal frequencies $\Omega:=\left(\Omega_{j}\right)_{j \in \mathbb{Z} \backslash \mathcal{I}}$ depend on $n$-parameters

$$
\xi \in \mathcal{O} \subset \mathbb{R}^{n} .
$$

For each $\xi$ there is an invariant $n$-torus $\mathcal{T}_{0}=\mathbb{T}^{n} \times\{0\} \times\{0\} \times\{0\}$ with frequency $\omega(\xi)$. In its normal space, the origin $(z, \bar{z})=0$ is an elliptic fixed point with proper frequencies $\Omega(\xi)$. The aim is to prove the persistence of a large portion of this family of linearly stable tori under small analytic perturbations $H=\mathcal{N}+P$.

- (A1) Parameter dependence. The map $\omega: \mathcal{O} \rightarrow \mathbb{R}^{n}, \xi \mapsto \omega(\xi)$, is Lipschitz continuous. With in mind the application to NLW we assume

- (A2) Frequency asymptotics. We have

$$
\Omega_{j}(\xi)=\sqrt{j^{2}+\mathrm{m}}+a(\xi) \in \mathbb{R}, \quad j \in \mathbb{Z} \backslash \mathcal{I},
$$

for some Lipschitz continuous functions $a(\xi) \in \mathbb{R}$.

By (A1) and (A2), the Lipschitz semi-norms of the frequency maps satisfy, for some $1 \leq M_{0}<\infty$,

$$
|\omega|^{\text {lip }},|\Omega|_{\infty}^{\text {lip }} \leq M_{0}
$$

where the Lipschitz semi-norm is

$$
|\Omega|_{\infty}^{\operatorname{lip}}:=|\Omega|_{\infty, \mathcal{O}}^{\operatorname{lip}}:=\sup _{\xi, \eta \in \mathcal{O}, \xi \neq \eta} \frac{|\Omega(\xi)-\Omega(\eta)|_{\infty}}{|\xi-\eta|}
$$


and $|z|_{\infty}:=\sup _{j \in \mathbb{Z} \backslash \mathcal{I}}\left|z_{j}\right|$. Note that by the Kirszbraun theorem (see e.g., [23]) applied componentwise we can extend $\omega, \Omega$ on the whole $\mathbb{R}^{n}$ with the same bound (4.3).

- (A3) Regularity. The perturbation $P: D(s, r) \times \mathcal{O} \rightarrow \mathbb{C}$ is $\lambda$-regular (see Definition 2.8).

In order to obtain the asymptotic expansion (4.9) for the perturbed frequencies we also assume

- (A4) Quasi-Töplitz. The perturbation $P$ (preserves momentum and) is quasi-Töplitz (see Definition 3.4).

Thanks to the conservation of momentum we restrict to the set of indices

$$
\begin{aligned}
\mathbf{I}:=\{ & (k, l) \in \mathbb{Z}^{n} \times \mathbb{Z}^{\infty},(k, l) \neq(0,0),|l| \leq 2, \text { where } \\
& \text { or } l=0, k \cdot \mathrm{j}=0, \\
& \text { or } l=\sigma e_{m}, m \in \mathbb{Z} \backslash \mathcal{I}, k \cdot \mathrm{j}+\sigma m=0, \\
& \text { or } \left.l=\sigma e_{m}+\sigma^{\prime} e_{n}, m, n \in \mathbb{Z} \backslash \mathcal{I}, k \cdot \mathrm{j}+\sigma m+\sigma^{\prime} n=0\right\} .
\end{aligned}
$$

Let

$$
P=P_{00}(x)+\bar{P}(x, y, z, \bar{z}) \quad \text { where } \quad \bar{P}(x, 0,0,0)=0 .
$$

Theorem 4.1 (KAM theorem). - Suppose that $H=\mathcal{N}+P$ satisfies (A1)-(A4) with $s, r>0,1<\theta, \mu<6, N>0$. Let $\gamma>0$ be a small parameter and set

$\varepsilon:=\max \left\{\gamma^{-2 / 3}\left|X_{P_{00}}\right|_{s, r}^{\lambda}, \gamma^{-2 / 3}\left\|X_{P_{00}}\right\|_{s, r}, \gamma^{-1}\left|X_{\bar{P}}\right|_{s, r}^{\lambda}, \gamma^{-1}\|\bar{P}\|_{s, r, N, \theta, \mu}^{T}\right\}, \quad \lambda:=\gamma / M_{0}$.

If $\varepsilon$ is small enough, then there exist:

- (Frequencies) Lipschitz functions $\omega^{\infty}: \mathbb{R}^{n} \rightarrow \mathbb{R}^{n}, \Omega^{\infty}: \mathbb{R}^{n} \rightarrow \ell_{\infty}, a_{ \pm}^{\infty}: \mathbb{R}^{n} \rightarrow \mathbb{R}$, such that

$$
\begin{gathered}
\left|\omega^{\infty}-\omega\right|+\lambda\left|\omega^{\infty}-\omega\right|^{\text {lip }}, \quad\left|\Omega^{\infty}-\Omega\right|_{\infty}+\lambda\left|\Omega^{\infty}-\Omega\right|_{\infty}^{\text {lip }} \leq C \gamma \varepsilon, \quad\left|a_{ \pm}^{\infty}\right| \leq C \gamma \varepsilon, \\
\sup _{\xi \in \mathbb{R}^{n}}\left|\Omega_{j}^{\infty}(\xi)-\Omega_{j}(\xi)-a_{\mathbf{s}(j)}^{\infty}(\xi)\right| \leq \gamma^{2 / 3} \varepsilon \frac{C}{|j|}, \quad \forall|j| \geq C_{\star} \gamma^{-1 / 3} .
\end{gathered}
$$

- (KAM normal form) A Lipschitz family of analytic symplectic maps

$$
\Phi: D(s / 4, r / 4) \times \mathcal{O}_{\infty} \ni\left(x_{\infty}, y_{\infty}, w_{\infty} ; \xi\right) \mapsto(x, y, w) \in D(s, r)
$$

close to the identity where

$$
\begin{aligned}
& \mathcal{O}_{\infty}:=\left\{\xi \in \mathcal{O}:\left|\omega^{\infty}(\xi) \cdot k+\Omega^{\infty}(\xi) \cdot l\right| \geq \frac{2 \gamma}{1+|k|^{\tau}}, \forall(k, l) \in \mathbf{I}\right. \text { defined in (4.5), } \\
&\left|\omega^{\infty}(\xi) \cdot k+p\right| \geq \frac{2 \gamma^{2 / 3}}{1+|k|^{\tau}}, \forall k \in \mathbb{Z}^{n}, p \in \mathbb{Z},(k, p) \neq(0,0), \tau>1 / b \text { see (3.2), } \\
&\left.\quad|\omega(\xi) \cdot k| \geq \frac{2 \gamma^{2 / 3}}{1+|k|^{n}}, \forall 0<|k|<\gamma^{-1 /(7 n)}\right\}
\end{aligned}
$$

such that, $\forall \xi \in \mathcal{O}_{\infty}$ :

$$
H^{\infty}(\cdot ; \xi):=H \circ \Phi(\cdot ; \xi)=\omega^{\infty}(\xi) \cdot y_{\infty}+\Omega^{\infty}(\xi) \cdot z_{\infty} \bar{z}_{\infty}+P^{\infty} \quad \text { has } \quad P_{\leq 2}^{\infty}=0 .
$$


Then, $\forall \xi \in \mathcal{O}_{\infty}$, the map $x_{\infty} \mapsto \Phi\left(x_{\infty}, 0,0 ; \xi\right)$ is a real analytic embedding of an elliptic, $n$-dimensional torus with frequency $\omega^{\infty}(\xi)$ for the system with Hamiltonian $H$.

The main novelty of Theorem 4.1 is the asymptotic decay (4.9) of the perturbed frequencies. In order to prove (4.9) we use the quasi-Töplitz property (A4) of the perturbation. The reason for introducing in (4.7) conditions for both the Lipschitz-sup and the Töplitz-norms is the following. For the measure estimates, we need the usual Lipschitz dependence of the perturbed frequencies with respect to the parameters, see (4.8). This is derived as in [27] and [2]. On the other hand, we do not need (in Section 6) a Lipschitz estimate on $a_{ \pm}^{\infty}$ (that, in any case, could be obtained). For this reason, we do not introduce the Lipschitz dependence in the Töplitz norm.

In the next Theorem 4.2 we verify the second order Melnikov non-resonance conditions thanks to

1. the asymptotic decay (4.9) of the perturbed frequencies,

2. the restriction to indices $(k, l) \in \mathbf{I}$ in (4.11) which follows by momentum conservation, see (A4).

As in [2], the Cantor set of "good" parameters $\mathcal{O}_{\infty}$ in (4.11), is expressed in terms of the final frequencies $\omega^{\infty}(\xi), \Omega^{\infty}(\xi)$ (and of the initial tangential frequencies $\omega(\xi)$ ) and not inductively as, for example, in [27]. This simplifies the measure estimates.

Theorem 4.2 (Measure estimate). - Let $\mathcal{O}:=[\rho / 2, \rho]^{n}, \rho>0$. Suppose

(4.13) $\omega(\xi)=\bar{\omega}+A \xi, \bar{\omega} \in \mathbb{R}^{n}, A \in \operatorname{Mat}(n \times n), \quad \Omega_{j}(\xi)=\sqrt{j^{2}+\mathrm{m}}+\vec{a} \cdot \xi, a \in \mathbb{R}^{n}$

and assume the non-degeneracy condition:

$$
A \text { invertible and } 2\left(A^{-1}\right)^{T} \vec{a} \notin \mathbb{Z}^{n} \backslash\{0\} .
$$

Then, the Cantor like set $\mathcal{O}_{\infty}$ defined in (4.11), with exponent

$$
\tau>\max \{2 n+1,1 / b\}
$$

(b is fixed in (3.2)), satisfies

$$
\left|\mathcal{O} \backslash \mathcal{O}_{\infty}\right| \leq C(\tau) \rho^{n-1} \gamma^{2 / 3}
$$

Theorem 4.2 is proved in Section 6. The asymptotic estimate (4.9) is used for the key inclusion (6.12).

\section{Proof of the KAM Theorem 4.1}

In the following by $a \lessdot b$ we mean that there exists $c>0$ depending only on $n, \mathrm{~m}, \kappa$ such that $a \leq c b$. 


\subsection{First step}

We perform a preliminary change of variables to improve the smallness conditions. For all $\xi$ in

$$
\mathcal{O}_{*}:=\left\{\xi \in \mathcal{O}:|\omega(\xi) \cdot k| \geq \frac{\gamma^{2 / 3}}{1+|k|^{n}}, \forall 0<|k|<\gamma^{-1 /(7 n)}\right\}
$$

we consider the solution

$$
F_{00}(x):=\sum_{0<|k|<\gamma^{-1 /(7 n)}} \frac{P_{00, k}}{\mathrm{i} \omega(\xi) \cdot k} e^{\mathrm{i} k \cdot x}
$$

of the homological equation

$$
-\operatorname{ad}_{\mathcal{N}} F_{00}+\Pi_{|k|<\gamma^{-1 /(7 n)}} P_{00}(x)=\left\langle P_{00}\right\rangle .
$$

Here $P_{00}$ is defined in (4.6) and $\langle\cdot\rangle$ denotes the mean value on the angles. Note that for any function $F_{00}(x)$ we have $\left\|F_{00}\right\|_{s, r}^{T}=\left\|X_{F_{00}}\right\|_{s, r}$, see Definition 3.4. We want to apply Proposition 3.2 with $s, r, s^{\prime}, r^{\prime} \rightsquigarrow 3 s / 4,3 r / 4, s / 2, r / 2$. The condition (3.63) is verified because

$$
\left\|F_{00}\right\|_{3 s / 4, r}^{T}=\left\|X_{F_{00}}\right\|_{3 s / 4, r} \stackrel{(5.2),(5.1),(2.55)}{\leq} C(n, s) \gamma^{-2 / 3}\left\|X_{P_{00}}\right\|_{s, r} \stackrel{(4.7)}{\leq} C(n, s) \varepsilon
$$

and $\varepsilon$ is sufficiently small. Hence the time-one flow

$$
\Phi_{00}:=\Phi_{F_{00}}^{1}: D\left(s_{0}, r_{0}\right) \times \mathcal{O}_{*} \rightarrow D(s, r) \quad \text { with } \quad s_{0}:=s / 2, r_{0}:=r / 2,
$$

is well defined, analytic, symplectic. Let $\mu_{0}<\mu, \theta_{0}>\theta, N_{0}>N$ large enough, so that (3.65) is satisfied with $s, r, N_{0}, \theta, \mu, \rightsquigarrow s, r, N, \theta, \mu$ and $s^{\prime}, r^{\prime}, N_{0}^{\prime}, \theta^{\prime}, \mu^{\prime} \rightsquigarrow s_{0}, r_{0}, N_{0}, \theta_{0}, \mu_{0}$. Note that here $N_{0}$ is independent of $\gamma$. Hence (3.66) implies

$$
\left\|e^{\operatorname{ad}_{F_{00}} \bar{P}}\right\|_{s_{0}, r_{0}, N_{0}, \theta_{0}, \mu_{0}}^{T} \leq 2\|\bar{P}\|_{s, r, N, \theta, \mu}^{T} .
$$

Noting that $e^{\operatorname{ad}_{F_{00}}} P_{00}=P_{00}$ and $e^{\operatorname{ad}_{F_{00}}} \mathcal{N}=\mathcal{N}+\operatorname{ad}_{F_{00}} \mathcal{N}$ the new Hamiltonian is

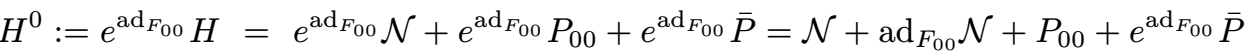

$$
\begin{aligned}
& \stackrel{(5.3)}{=}\left(\left\langle P_{00}\right\rangle+\mathcal{N}\right)+\left(\Pi_{|k| \geq \gamma^{-1 /(7 n)}} P_{00}+e^{\operatorname{ad}_{F 00} \bar{P}}\right)=: \mathcal{N}_{0}+P_{0} \text {. }
\end{aligned}
$$

By (2.57) (and since $P_{00}(x)$ depends only on $x$ )

$$
\left\|\Pi_{|k| \geq \gamma^{-1 /(7 n)}} P_{00}\right\|_{3 s / 4, r}^{T} \leq 4 e^{-s \gamma^{-1 /(7 n)} / 4}\left\|X_{P_{00}}\right\|_{s, r} \stackrel{(4.7)}{\leq} 4 \gamma^{2 / 3} e^{-s \gamma^{-1 /(7 n)} / 4} \varepsilon \leq \gamma \varepsilon,
$$

for $\gamma$ small. By (5.7), (5.5) and (4.7) we get

$$
\left\|P_{0}\right\|_{s_{0}, r_{0}, N_{0}, \theta_{0}, \mu_{0}}^{T}<3 \gamma \varepsilon .
$$

In the same way, since $\left|X_{F_{00}}\right|_{3 s / 4, r}^{\lambda} \leq C(n, s) \gamma^{-2 / 3}\left|X_{P_{00}}\right|_{s, r}^{\lambda}$, we also obtain the Lipschitz estimate

$$
\left|X_{P_{0}}\right|_{s_{0}, r_{0}}^{\lambda}<3 \gamma \varepsilon
$$

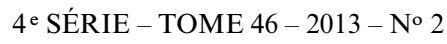




\subsection{KAM step}

We now consider the generic KAM step for an Hamiltonian

$$
H=\mathcal{N}+P=\mathcal{N}+P_{\bar{K}}^{\leq 2}+\left(P-P_{\bar{K}}^{\leq 2}\right)
$$

where $P_{\bar{K}}^{\leq 2}$ are defined as in (3.38).

\subsubsection{Homological equation. -}

Lemma 5.1. - Assume that

$$
\left|\Omega_{j}-\sqrt{j^{2}+\mathrm{m}}-a_{\mathrm{s}(j)}\right| \leq \frac{\gamma}{|j|}, \quad \forall|j| \geq j_{*},
$$

for some $a_{+}, a_{-} \in \mathbb{R}$. Let $\Delta_{k, m, n}:=\omega \cdot k+\Omega_{m}-\Omega_{n}, \tilde{\Delta}_{k, m, n}:=\omega \cdot k+|m|-|n|$.

If $|m|,|n| \geq \max \left\{j_{*}, \sqrt{\mathrm{m}}\right\}$ and $\mathbf{s}(m)=\mathbf{s}(n)$, then

$$
\left|\Delta_{k, m, n}-\tilde{\Delta}_{k, m, n}\right| \leq \frac{\mathrm{m}}{2} \frac{|m-n|}{|n||m|}+\gamma\left(\frac{1}{|m|}+\frac{1}{|n|}\right)+\frac{\mathrm{m}^{2}}{2}\left(\frac{1}{|m|^{3}}+\frac{1}{|n|^{3}}\right) .
$$

Proof. - For $0 \leq x \leq 1$ we have $|\sqrt{1+x}-1-x / 2| \leq x^{2} / 2$. Setting $x:=\mathrm{m} / n^{2}$ (which is $\leq 1$ ) and using (5.11), we get

$$
\left|\Omega_{n}-\right| n\left|-\frac{\mathrm{m}}{2|n|}-a_{\mathrm{s}(n)}\right| \leq \frac{\gamma}{|n|}+\frac{\mathrm{m}^{2}}{2|n|^{3}} .
$$

An analogous estimate holds for $\Omega_{m}$. Since $\left|\Delta_{k, m, n}-\tilde{\Delta}_{k, m, n}\right|=\left|\Omega_{m}-\right| m\left|-\Omega_{n}+\right| n||$ the estimate (5.12) follows noting that $a_{\mathbf{s}(m)}=a_{\mathbf{s}(n)}$.

For a monomial $\mathfrak{m}_{k, i, \alpha, \beta}:=e^{\mathrm{i} k \cdot x} y^{i} z^{\alpha} \bar{z}^{\beta}$ we set

$$
\left[\mathfrak{m}_{k, i, \alpha, \beta}\right]:= \begin{cases}\mathfrak{m}_{k, i, \alpha, \beta} & \text { if } \quad k=0, \alpha=\beta \\ 0 & \text { otherwise. }\end{cases}
$$

The following key proposition proves that the solution of the homological equation with a quasi-Töplitz datum is quasi-Töplitz.

Proposition 5.1 (Homological equation). - Let $K \in \mathbb{N}$. For all $\xi \in \mathcal{O}$ such that

$$
|\omega(\xi) \cdot k+\Omega(\xi) \cdot l| \geq \frac{\gamma}{\langle k\rangle^{\tau}}, \forall(k, l) \in \mathbf{I}(\text { see }(4.5)),|k| \leq K
$$

then $\forall P_{K}^{(h)} \in \mathcal{H}_{s, r}^{\text {null }}, h=0,1,2$ (see (3.36), (3.37)), the homological equations

$$
-\operatorname{ad}_{\mathcal{N}} F_{K}^{(h)}+P_{K}^{(h)}=\left[P_{K}^{(h)}\right], \quad h=0,1,2,
$$

have a unique solution of the same form $F_{K}^{(h)} \in \mathcal{H}_{s, r}^{\text {null }}$ with $\left[F_{K}^{(h)}\right]=0$ and

$$
\left\|X_{F_{K}^{(h)}}\right\|_{s, r}<\gamma^{-1} K^{\tau}\left\|X_{P_{K}^{(h)}}\right\|_{s, r}, \quad\left|X_{F_{K}^{(h)}}\right|_{s, r}^{\lambda} \lessdot \gamma^{-1} K^{\tau+1}\left|X_{P_{K}^{(h)}}\right|_{s, r}^{\lambda}
$$

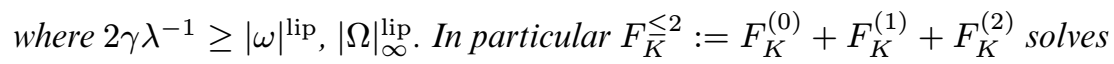

$$
-\operatorname{ad}_{\mathcal{N}} F_{\bar{K}}^{\leq 2}+P_{\bar{K}}^{\leq 2}=\left[P_{\bar{K}}^{\leq 2}\right] .
$$

Assume now that $P_{K}^{(h)} \in \mathcal{Q}_{s, r}^{T}\left(N_{0}, \theta, \mu\right)$ and $\Omega(\xi)$ satisfies (5.11) for all $|j| \geq \theta N_{0}^{*}$ where

$$
N_{0}^{*}:=\max \left\{N_{0}, \hat{c} \gamma^{-1 / 3} K^{\tau+1}\right\}
$$


for a constant $\hat{c}:=\hat{c}(\mathrm{~m}, \kappa) \geq 1$. Then, $\forall \xi \in \mathcal{O}$ such that

$$
|\omega(\xi) \cdot k+p| \geq \frac{\gamma^{2 / 3}}{\langle k\rangle^{\tau}}, \forall|k| \leq K, p \in \mathbb{Z}
$$

we have $F_{K}^{(h)} \in \mathcal{Q}_{s, r}^{T}\left(N_{0}^{*}, \theta, \mu\right), h=0,1,2$, and

$$
\left\|F_{K}^{(h)}\right\|_{s, r, N_{0}^{*}, \theta, \mu}^{T} \leq 4 \hat{c} \gamma^{-1} K^{2 \tau}\left\|P_{K}^{(h)}\right\|_{s, r, N_{0}, \theta, \mu}^{T} .
$$

Proof. - The solution of the homological Equation (5.15) is

$$
F_{K}^{(h)}:=-\mathrm{i} \sum_{\substack{|k| \leq K,(k, i, \alpha, \beta) \neq(0, i, \alpha, \alpha) \\ 2 i+|\alpha|+|\beta|=h}} \frac{P_{k, i, \alpha, \beta}}{\Delta_{k, i, \alpha, \beta}} e^{\mathrm{i} k \cdot x} y^{i} z^{\alpha} \bar{z}^{\beta}, \quad \Delta_{k, i, \alpha, \beta}:=\omega(\xi) \cdot k+\Omega(\xi) \cdot(\alpha-\beta) .
$$

The divisors $\Delta_{k, i, \alpha, \beta} \neq 0, \forall(k, i, \alpha, \beta) \neq(0, i, \alpha, \alpha)$, because $(k, i, \alpha, \beta) \neq(0, i, \alpha, \alpha)$ is equivalent to $(k, \alpha-\beta) \in \mathbf{I}$, and the bounds (5.14) hold. Then the first estimates in (5.16) follows by Lemma 2.18. The Lipsichtz estimate in (5.16) is standard, see e.g., Lemma 1 (and the first comment after the statement) of [27]. We just note that the Melnikov condition used in [27] follows by (5.14) and momentum consevation, e.g.,

$$
\left|\omega \cdot k+\Omega_{m}-\Omega_{n}\right| \stackrel{(5.14)}{\geq} \frac{\gamma}{\langle k\rangle^{\tau}} \stackrel{(5.22)}{=} \frac{\gamma|m-n|}{|\mathrm{j} \cdot k|\langle k\rangle^{\tau}} \geq \gamma \frac{|m-n|}{\kappa\langle k\rangle^{\tau+1}} .
$$

For the Töplitz estimate notice that the cases $h=0,1$ are trivial since $\Pi_{N, \theta, \mu} F_{K}^{\leq 1}=0$. When $h=2$ we first consider the subtlest case when $P_{K}^{(2)}$ contains only the monomials with $i=0$, $|\alpha|=|\beta|=1$ (see (3.36)), namely

$$
\mathcal{P}:=P_{K}^{(2)}=\sum_{|k| \leq K, m, n \in \mathbb{Z} \backslash \mathcal{I}} P_{k, m, n} e^{\mathrm{i} k \cdot x} z_{m} \bar{z}_{n},
$$

and, because of the conservation of momentum, the indices $k, m, n$ in (5.21) are restricted to

$$
\mathrm{j} \cdot k+m-n=0 .
$$

The unique solution $F_{K}^{(2)}$ of (5.15) with $\left[F_{K}^{(2)}\right]=0$ is

$$
\mathcal{F}:=F_{K}^{(2)}:=-\mathrm{i} \sum_{|k| \leq K,(k, m, n) \neq(0, m, m)} \frac{P_{k, m, n}}{\Delta_{k, m, n}} e^{\mathrm{i} k \cdot x} z_{m} \bar{z}_{n}, \Delta_{k, m, n}:=\omega(\xi) \cdot k+\Omega_{m}(\xi)-\Omega_{n}(\xi) .
$$

Note that by (5.14) and (5.22) we have $\Delta_{k, m, n} \neq 0$ if and only if $(k, m, n) \neq(0, m, m)$.

Let us prove (5.20). For all $N \geq N_{0}^{*}$

$$
\Pi_{N, \theta, \mu} \mathcal{F}=-\mathrm{i} \sum_{|k| \leq K,|m|,|n|>\theta N} \frac{P_{k, m, n}}{\Delta_{k, m, n}} e^{\mathrm{i} k \cdot x} z_{m} \bar{z}_{n},
$$

and note that $e^{\mathrm{i} k \cdot x}$ is $(N, \mu)$-low momentum since $|k| \leq K<\left(N_{0}^{*}\right)^{b} \leq N^{b}$ by (5.18) and $\tau>1 / b$. By assumption $\mathcal{P} \in \mathcal{Q}_{s, r, N_{0}, \theta, \mu}^{T}$ and so, recalling Formula (3.45), we may write, $\forall N \geq N_{0}^{*} \geq N_{0}$

$$
\Pi_{N, \theta, \mu} \mathcal{P}=\tilde{\mathcal{P}}+N^{-1} \hat{\mathcal{P}} \quad \text { with } \quad \tilde{\mathcal{P}}:=\sum_{|k| \leq K,|m|,|n|>\theta N} \tilde{P}_{k, m-n} e^{\mathrm{i} k \cdot x} z_{m} \bar{z}_{n} \in \mathcal{T}_{s, r}(N, \theta, \mu)
$$


and

$$
\left\|X_{\mathcal{P}}\right\|_{s, r},\left\|X_{\tilde{\mathcal{P}}}\right\|_{s, r},\left\|X_{\hat{\mathcal{P}}}\right\|_{s, r} \leq 2\|\mathcal{P}\|_{s, r}^{T}
$$

We now prove that

$$
\tilde{\mathcal{F}}:=\sum_{|k| \leq K,|m|,|n|>\theta N} \frac{\tilde{P}_{k, m-n}}{\tilde{\Delta}_{k, m, n}} e^{\mathrm{i} k \cdot x} z_{m} \bar{z}_{n}, \quad \tilde{\Delta}_{k, m, n}:=\omega(\xi) \cdot k+|m|-|n|,
$$

is a Töplitz approximation of $\mathcal{F}$. Since $|m|,|n|>\theta N \geq \theta N_{0}^{*}>N_{0}^{*} \stackrel{(5.18)}{>} \kappa K \geq|\mathrm{j} \cdot k|$ by (3.1), we deduce by (5.22) that $m, n$ have the same sign. Then

$$
\tilde{\Delta}_{k, m, n}=\omega(\xi) \cdot k+|m|-|n|=\omega(\xi) \cdot k+\mathbf{s}(m)(m-n), \quad \mathbf{s}(m):=\operatorname{sign}(m),
$$

and $\tilde{\mathcal{F}}$ in (5.27) is $(N, \theta, \mu)$-Töplitz (see (3.15)). Moreover, since $|m|-|n| \in \mathbb{Z}$, by (5.19), we get

$$
\left|\tilde{\Delta}_{k, m, n}\right| \geq \gamma^{2 / 3}\langle k\rangle^{-\tau}, \quad \forall|k| \leq K, m, n,
$$

and Lemma 2.18 and (5.27) imply

$$
\left\|X_{\tilde{\mathcal{F}}}\right\|_{s, r} \leq \gamma^{-2 / 3} K^{\tau}\left\|X_{\tilde{\mathcal{P}}}\right\|_{s, r}
$$

The Töplitz defect is

$$
\begin{aligned}
N^{-1} \hat{\mathcal{F}} & :=\Pi_{N, \theta, \mu} \mathcal{F}-\tilde{\mathcal{F}} \\
& \stackrel{(5.24),(5.27)}{=} \sum_{|k| \leq K,|m|,|n|>\theta N}\left(\frac{P_{k, m, n}}{\Delta_{k, m, n}}-\frac{\tilde{P}_{k, m-n}}{\tilde{\Delta}_{k, m, n}}\right) e^{\mathrm{i} k \cdot x} z_{m} \bar{z}_{n} \\
& =\sum_{|k| \leq K,|m|,|n|>\theta N}\left[\left(\frac{P_{k, m, n}}{\Delta_{k, m, n}}-\frac{P_{k, m, n}}{\tilde{\Delta}_{k, m, n}}\right)+\left(\frac{P_{k, m, n}-\tilde{P}_{k, m-n}}{\tilde{\Delta}_{k, m, n}}\right)\right] e^{\mathrm{i} k \cdot x} z_{m} \bar{z}_{n} \\
& \stackrel{(5.25)}{=} \sum_{|k| \leq K,|m|,|n|>\theta N}\left[P_{k, m, n}\left(\frac{\tilde{\Delta}_{k, m, n}-\Delta_{k, m, n}}{\Delta_{k, m, n} \tilde{\Delta}_{k, m, n}}\right)+N^{-1} \frac{\hat{P}_{k, m, n}}{\tilde{\Delta}_{k, m, n}}\right] e^{\mathrm{i} k \cdot x} z_{m} \bar{z}_{n} .
\end{aligned}
$$

By (5.12), $|m|,|n| \geq \theta N \geq N$, and $|m-n| \leq \kappa K$ (see (5.22)) we get, taking $\hat{c}$ large enough, $(5.31)$

$$
\left|\tilde{\Delta}_{k, m, n}-\Delta_{k, m, n}\right| \leq \frac{\mathrm{m} \kappa K}{2 N^{2}}+\frac{2 \gamma}{N}+\frac{\mathrm{m}^{2}}{N^{3}} \leq \frac{\hat{c}}{4 N}\left(\frac{K}{N}+\gamma\right) \stackrel{(5.18)}{\leq} \min \left\{\frac{\hat{c} \gamma^{1 / 3}}{2 N}, \frac{\gamma^{2 / 3}}{2 K^{\tau}}\right\} .
$$

Hence

$$
\left|\Delta_{k, m, n}\right| \geq\left|\tilde{\Delta}_{k, m, n}\right|-\left|\tilde{\Delta}_{k, m, n}-\Delta_{k, m, n}\right| \stackrel{(5.28),(5.31)}{\geq} \frac{\gamma^{2 / 3}}{\langle k\rangle^{\tau}}-\frac{\gamma^{2 / 3}}{2 K^{\tau}} \geq \frac{\gamma^{2 / 3}}{2\langle k\rangle^{\tau}} .
$$

Therefore (5.31), (5.28), (5.32) imply

$$
\frac{\left|\tilde{\Delta}_{k, m, n}-\Delta_{k, m, n}\right|}{\left|\Delta_{k, m, n}\right|\left|\tilde{\Delta}_{k, m, n}\right|} \leq \frac{\hat{c} \gamma^{1 / 3}}{2 N} \frac{2\langle k\rangle^{\tau}}{\gamma^{2 / 3}} \frac{\langle k\rangle^{\tau}}{\gamma^{2 / 3}} \leq \frac{\hat{c}}{N \gamma} K^{2 \tau}
$$

and (5.30), (5.28), and Lemma 2.18, imply

$$
\left\|X_{\hat{\mathcal{F}}}\right\|_{s, r} \leq \hat{c} \gamma^{-1} K^{2 \tau}\left\|X_{\mathcal{P}}\right\|_{s, r}+\gamma^{-2 / 3} K^{\tau}\left\|X_{\hat{\mathcal{P}}}\right\|_{s, r} \stackrel{(5.26)}{\leq} 4 \hat{c} \gamma^{-1} K^{2 \tau}\|\mathcal{P}\|_{s, r}^{T} .
$$


In conclusion (5.16), (5.29), (5.33) prove (5.20) for $\mathcal{F}$.

Let us briefly discuss the case when $h=2$ and $P_{K}^{(2)}$ contains only the monomials with $i=0,|\alpha|=2,|\beta|=0$ or viceversa (see (3.36)). Denoting

$$
\mathcal{P}:=P_{K}^{(2)}:=\sum_{|k| \leq K, m, n \in \mathbb{Z} \backslash \mathcal{I}} P_{k, m, n} e^{\mathrm{i} k \cdot x} z_{m} z_{n},
$$

we have

$$
\Pi_{N, \theta, \mu} \mathcal{F}=-\mathrm{i} \sum_{|k| \leq K,|m|,|n|>\theta N} \frac{P_{k, m, n}}{\omega \cdot k+\Omega_{m}+\Omega_{n}} e^{\mathrm{i} k \cdot x} z_{m} z_{n}
$$

where $\left|\omega \cdot k+\Omega_{m}+\Omega_{n}\right|>(|m|+|n|) / 2>\theta N / 2$ since $|m|,|n|>\theta N$ and $|k| \leq K<N^{b}$. In this case we may take as Töplitz approximation $\tilde{\mathcal{F}}=0$.

5.2.2. The new Hamiltonian $H^{+}$. - Let $F=F_{\bar{K}}^{\leq 2}$ be the solution of the homological Equation (5.17). If, for $s / 2 \leq s_{+}<s, r / 2 \leq r_{+}<r$, the condition

$$
\|F\|_{s, r, N_{0}^{*}, \theta, \mu}^{T} \leq c(n) \delta_{+}, \quad \delta_{+}:=\min \left\{1-\frac{s_{+}}{s}, 1-\frac{r_{+}}{r}\right\}
$$

holds (see (3.63)), then Proposition 3.2 (with $s^{\prime} \rightsquigarrow s_{+}, r^{\prime} \rightsquigarrow r_{+}, N_{0} \rightsquigarrow N_{0}^{*}$ defined in (5.18)) implies that the Hamiltonian flow $\Phi_{F}^{1}: D\left(s_{+}, r_{+}\right) \rightarrow D(s, r)$ is well defined, analytic and symplectic. We transform the Hamiltonian $H$ in (5.10), obtaining

$$
\begin{aligned}
H^{+}:=e^{\operatorname{ad}_{F}} & \stackrel{(2.83)}{=} H+\operatorname{ad}_{F}(H)+\sum_{j \geq 2} \frac{1}{j !} \operatorname{ad}_{F}^{j}(H) \\
& \stackrel{(5.10)}{=} \mathcal{N}+P_{\bar{K}}^{\leq 2}+\left(P-P_{K}^{\leq 2}\right)+\operatorname{ad}_{F} \mathcal{N}+\operatorname{ad}_{F} P+\sum_{j \geq 2} \frac{1}{j !} \operatorname{ad}_{F}^{j}(H) \\
& \stackrel{(5.17)}{=} \mathcal{N}+\left[P_{\bar{K}}^{\leq 2}\right]+P-P_{\bar{K}}^{\leq 2}+\operatorname{ad}_{F} P+\sum_{j \geq 2} \frac{1}{j !} \operatorname{ad}_{F}^{j}(H):=\mathcal{N}^{+}+P^{+}
\end{aligned}
$$

with new normal form

$$
\mathcal{N}^{+}:=\mathcal{N}+\hat{\mathcal{N}}, \quad \hat{\mathcal{N}}:=\left[P_{\bar{K}}^{\leq 2}\right]=\hat{e}+\hat{\omega} \cdot y+\hat{\Omega} z \cdot \bar{z}
$$

$\hat{\omega}_{i}:=\partial_{y_{i} \mid y=0, z=\bar{z}=0}\langle P\rangle, i=1, \ldots n, \hat{\Omega}:=\left(\hat{\Omega}_{j}\right)_{j \in \mathbb{Z} \backslash \mathcal{I}}, \hat{\Omega}_{j}:=[P]_{j}:=\partial_{z_{j} \bar{z}_{j} \mid y=0, z=\bar{z}=0}^{2}\langle P\rangle$

(the \langle\rangle denotes the average with respect to the angles $x$ ) and new perturbation

$$
P^{+}:=P-P_{K}^{\leq 2}+\operatorname{ad}_{F} P^{\leq 2}+\operatorname{ad}_{F} P^{\geq 3}+\sum_{j \geq 2} \frac{1}{j !} \operatorname{ad}_{F}^{j}(H)
$$

having decomposed $P=P^{\leq 2}+P^{\geq 3}$ with $P^{\geq 3}:=\sum_{h \geq 3} P^{(h)}$, see (3.36).

\subsubsection{The new normal form $\mathcal{N}^{+}$}

Lemma 5.2. - Let $P \in \mathcal{Q}_{s, r}^{T}\left(N_{0}, \theta, \mu\right)$ with $1<\theta, \mu<6, N_{0} \geq 9$. Then

$$
|\hat{\omega}|,|\hat{\Omega}|_{\infty} \leq 2\left\|P^{(2)}\right\|_{s, r, N_{0}, \theta, \mu}^{T}
$$

and there exist $\hat{a}_{ \pm} \in \mathbb{R}$ satisfying

$$
\left|\hat{a}_{ \pm}\right| \leq 2\left\|P^{(2)}\right\|_{s, r, N_{0}, \theta, \mu}^{T}
$$

$4^{\mathrm{e}}$ SÉRIE - TOME $46-2013$ - No 2 
such that

$$
\left|\hat{\Omega}_{j}-\hat{a}_{\mathbf{s}(j)}\right| \leq \frac{40}{|j|}\left\|P^{(2)}\right\|_{s, r, N_{0}, \theta, \mu}^{T}, \quad \forall|j| \geq 6\left(N_{0}+1\right) .
$$

Moreover $|\hat{\omega}|^{\text {lip }},|\hat{\Omega}|_{\infty}^{\operatorname{lip}} \lessdot\left|X_{P^{(2)}}\right|_{s, r}^{\operatorname{lip}}$.

Lemma 5.2 is based on the following elementary lemma, whose proof is postponed.

Lemma 5.3. - Suppose that, $\forall N \geq N_{0} \geq 9, j \geq \theta N$,

$$
\Omega_{j}=a_{N}+b_{N, j} N^{-1} \text { with } a_{N}, b_{N, j} \in \mathbb{R},\left|a_{N}\right| \leq c_{1},\left|b_{N, j}\right| \leq c_{1},
$$

for some $c_{1}>0$ (independent of $j$ ). Then there exists $a \in \mathbb{R}$, satisfying $|a| \leq c_{1}$, such that

$$
\left|\Omega_{j}-a\right| \leq \frac{20 c_{1}}{|j|}, \quad \forall|j| \geq 6\left(N_{0}+1\right) .
$$

Proof of Lemma 5.2. - The estimate on $\hat{\omega}$ is trivial. Regarding $\hat{\Omega}$ we set (recall (3.36), $(3.42))$

$$
P_{0}^{(2)}:=\Pi_{k=0} \Pi_{|\alpha|=|\beta|=1} \Pi^{(2)} P=\sum_{j}[P]_{j} z_{j} \bar{z}_{j}
$$

since, by the momentum conservation (2.86), all the monomials in $P_{0}^{(2)}$ have $\alpha=\beta=e_{j}$. Note that $[P]_{j}$ is defined in (5.36). By Lemma 2.19

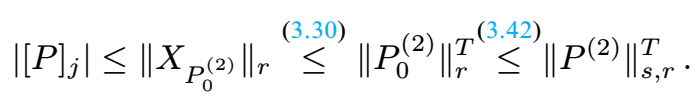

We now prove (5.39) for $j>0$ (the case $j<0$ is similar). Since $P_{0}^{(2)} \in \mathcal{Q}_{r}^{T}(N, \theta, \mu)$, for all $N \geq N_{0}$, we may write $\Pi_{N, \theta, \mu} P_{0}^{(2)}=\tilde{P}_{0, N}^{(2)}+N^{-1} \hat{P}_{0, N}^{(2)}$ with

$$
\tilde{P}_{0, N}^{(2)}:=\sum_{j>\theta N} \tilde{P}_{j} z_{j} \bar{z}_{j} \in \mathcal{T}_{r}(N, \theta, \mu), \quad \hat{P}_{0, N}^{(2)}:=\sum_{j>\theta N} \hat{P}_{j} z_{j} \bar{z}_{j}
$$

and

$$
\left\|X_{P_{0}^{(2)}}\right\|_{r},\left\|X_{\tilde{P}_{0, N}^{(2)}}\right\|_{r},\left\|X_{\hat{P}_{0, N}^{(2)}}\right\|_{r} \leq 2\left\|P_{0}^{(2)}\right\|_{r}^{T} \leq 2\left\|P^{(2)}\right\|_{s, r}^{T} .
$$

For $|j|>\theta N$, since all the quadratic forms in (5.43) are diagonal, we have

$$
\hat{\Omega}_{j}=[P]_{j}=\tilde{P}_{j}+N^{-1} \hat{P}_{j}:=a_{N,+}+N^{-1} b_{N, j}
$$

where $a_{N,+}:=\tilde{P}_{j}$ is independent of $j>0$ because $\tilde{P}_{0, N}^{(2)} \in \mathcal{T}_{r}(N, \theta, \mu)$ (see (3.15)). Applying Lemma 2.19 to $\tilde{P}_{0, N}^{(2)}$ and $\hat{P}_{0, N}^{(2)}$, we obtain

$$
\left|a_{N,+}\right| \leq\left\|X_{\tilde{P}_{0, N}^{(2)}}\right\|_{s, r} \stackrel{(5.43)}{\leq} 2\left\|P^{(2)}\right\|_{s, r}^{T}, \quad\left|b_{N, j}\right|=\left|\hat{P}_{j}\right| \leq\left\|X_{\hat{P}_{0, N}^{(2)}}\right\|_{r} \stackrel{(5.43)}{\leq} 2\left\|P^{(2)}\right\|_{r}^{T} .
$$

Hence the assumptions of Lemma 5.3 are satisfied with $c_{1}=2\left\|P^{(2)}\right\|_{s, r}^{T}$ and (5.39) follows.

The final Lipschitz estimate is standard, see e.g., [2], [27]. 
Proof of Lemma 5.3. - For all $N_{1}>N \geq N_{0}, j \geq \theta N_{1}$ we get, by (5.40),

$$
\left|a_{N}-a_{N_{1}}\right|=\left|b_{N_{1}, j} N_{1}^{-1}-b_{N, j} N^{-1}\right| \leq 2 c_{1} N^{-1} .
$$

Therefore $a_{N}$ is a Cauchy sequence. Let $a:=\lim _{N \rightarrow+\infty} a_{N}$ be its limit. Since $\left|a_{N}\right| \leq c_{1}$ we have $|a| \leq c_{1}$. Moreover, letting $N_{1} \rightarrow+\infty$ in (5.44), we derive $\left|a-a_{N}\right| \leq 2 c_{1} N^{-1}$, $\forall N \geq N_{0}$, and, using also (5.40),

$$
\left|\Omega_{j}-a\right| \leq\left|\Omega_{j}-a_{N}\right|+\left|a_{N}-a\right| \leq 3 c_{1} N^{-1}, \quad \forall N \geq N_{0}, j \geq 6 N .
$$

For all $j \geq 6\left(N_{0}+1\right)$ let $N:=[j / 6]$ (where [.] denotes the integer part). Since $N \geq N_{0}$, $j \geq 6 N$,

$$
\left|\Omega_{j}-a\right| \stackrel{(5.45)}{\leq} \frac{3 c_{1}}{[j / 6]} \leq \frac{3 c_{1}}{(j / 6)-1} \leq \frac{18 c_{1}}{j}\left(1+\frac{1}{N_{0}}\right) \leq \frac{20 c_{1}}{j}
$$

for all $j \geq 6\left(N_{0}+1\right)$.

5.2.4. The new perturbation $P^{+}$. - We introduce, for $h=0,1,2$,

$$
\begin{aligned}
& \varepsilon^{(h)}:=\gamma^{-1} \max \left\{\left\|P^{(h)}\right\|_{s, r, N_{0}, \theta, \mu}^{T},\left|X_{P^{(h)}}\right|_{s, r}^{\lambda}\right\}, \quad \bar{\varepsilon}:=\sum_{h=0}^{2} \varepsilon^{(h)}, \\
& \Theta:=\gamma^{-1} \max \left\{\|P\|_{s, r, N_{0}, \theta, \mu}^{T},\left|X_{P}\right|_{s, r}^{\lambda}\right\}
\end{aligned}
$$

( $\lambda$ defined in (4.7)) and the corresponding quantities for $P^{+}$with indices $r_{+}, s_{+}, N_{0}^{+}, \theta_{+}, \mu_{+}$. The $P^{(h)}$ denote the homogeneous components of $P$ of degree $h$ (see (3.36)).

Proposition 5.2 (KAM step). - Suppose $\left(s, r, N_{0}, \theta, \mu\right),\left(s_{+}, r_{+}, N_{0}^{+}, \theta_{+}, \mu_{+}\right)$satisfy $s / 2 \leq s_{+}<s, r / 2 \leq r_{+}<r$,

$$
\begin{gathered}
N_{0}^{+}>\max \left\{N_{0}^{*}, \bar{N}\right\}(\text { recall }(5.18),(3.64)), \quad 2\left(N_{0}^{+}\right)^{-b} \ln ^{2} N_{0}^{+} \leq b\left(s-s_{+}\right), \\
\kappa\left(N_{0}^{+}\right)^{b-L} \ln N_{0}^{+} \leq \mu-\mu_{+}, \quad(6+\kappa)\left(N_{0}^{+}\right)^{L-1} \ln N_{0}^{+} \leq \theta_{+}-\theta .
\end{gathered}
$$

Assume that

$$
\bar{\varepsilon} K^{\bar{\tau}} \delta_{+}^{-1} \leq c \text { small enough }, \quad \Theta \leq 1,
$$

where $\bar{\tau}:=2 \tau+n+1$ and $\delta_{+}$is defined in (5.35). Suppose also that (5.11) holds for $|j| \geq \theta N_{0}^{*}$.

Then, for all $\xi \in \mathcal{O}$ satisfying (5.14),(5.19), denoting by $F:=F_{\bar{K}}^{\leq 2}$ the solution of the homological Equation (5.17), the Hamiltonian flow $\Phi_{F}^{1}: D\left(s_{+}, r_{+}\right) \rightarrow D(s, r)$, and the transformed Hamiltonian

$$
H^{+}:=H \circ \Phi_{F}^{1}=e^{\operatorname{ad}_{F}} H=\mathcal{N}_{+}+P_{+}
$$

satisfies

$$
\begin{gathered}
\varepsilon_{+}^{(0)} \lessdot \delta_{+}^{-2} K^{2 \bar{\tau}} \bar{\varepsilon}^{2}+\varepsilon^{(0)} e^{-\left(s-s_{+}\right) K} \\
\varepsilon_{+}^{(1)} \lessdot \delta_{+}^{-2} K^{2 \bar{\tau}}\left(\varepsilon^{(0)}+\bar{\varepsilon}^{2}\right)+\varepsilon^{(1)} e^{-\left(s-s_{+}\right) K} \\
\varepsilon_{+}^{(2)} \lessdot \delta_{+}^{-2} K^{2 \bar{\tau}}\left(\varepsilon^{(0)}+\varepsilon^{(1)}+\bar{\varepsilon}^{2}\right)+\varepsilon^{(2)} e^{-\left(s-s_{+}\right) K} \\
\Theta_{+} \leq \Theta\left(1+C \delta_{+}^{-2} K^{2 \bar{\tau}} \bar{\varepsilon}\right) .
\end{gathered}
$$


We focus on the quasi-Töplitz estimates, the Lipschitz ones follow formally in the same way. The proof splits in several lemmas where we analyze each term of $P^{+}$in (5.37). We note first that

$$
\left\|P_{\bar{K}}^{\leq 2}\right\|_{s, r, N_{0}, \theta, \mu}^{T} \stackrel{(3.41)}{\leq}\left\|P^{\leq 2}\right\|_{s, r, N_{0}, \theta, \mu}^{T \stackrel{(3.38),(5.46)}{\leq}} \gamma \bar{\varepsilon} .
$$

Moreover, the solution $F=F^{(0)}+F^{(1)}+F^{(2)}$ of the homological Equation (5.17) (for brevity $F^{(h)} \equiv F_{K}^{(h)}$ and $F \equiv F_{K}^{\leq 2}$ ) satisfies, by (5.20) (with $N_{0}^{*}$ defined in (5.18)), (3.41), (5.46),

$$
\left\|F^{(h)}\right\|_{s, r, N_{0}^{*}, \theta, \mu}^{T} \lessdot K^{\bar{\tau}} \varepsilon^{(h)}, h=0,1,2, \quad\|F\|_{s, r, N_{0}^{*}, \theta, \mu}^{T} \lessdot K^{\bar{\tau}} \bar{\varepsilon} .
$$

Hence (5.49) and (5.53) imply condition (5.35) and therefore $\Phi_{F}^{1}: D\left(s_{+}, r_{+}\right) \rightarrow D(s, r)$ is well defined. We now estimate the terms of the new perturbation $P^{+}$in (5.37).

Lemma 5.4. -

$$
\left\|\operatorname{ad}_{F}\left(P^{\leq 2}\right)\right\|_{s_{+}, r_{+}, N_{0}^{+}, \theta_{+}, \mu_{+}}^{T}+\left\|\sum_{j \geq 2} \frac{1}{j !} \operatorname{ad}_{F}^{j}(H)\right\|_{s_{+}, r_{+}, N_{0}^{+}, \theta_{+}, \mu_{+}}^{T} \lessdot \delta_{+}^{-2} \gamma K^{2 \bar{\tau}} \bar{\varepsilon}^{2} .
$$

Proof. - We have

$$
\begin{gathered}
\sum_{j \geq 2} \frac{1}{j !} \operatorname{ad}_{F}^{j}(H)=\sum_{j \geq 2} \frac{1}{j !} \operatorname{ad}_{F}^{j}(\mathcal{N}+P)=\sum_{j \geq 2} \frac{1}{j !} \operatorname{ad}_{F}^{j-1}\left(\operatorname{ad}_{F} \mathcal{N}\right)+\sum_{j \geq 2} \frac{1}{j !} \operatorname{ad}_{F}^{j}(P) \\
\stackrel{(5.17)}{=} \sum_{j \geq 2} \frac{1}{j !} \operatorname{ad}_{F}^{j-1}\left(\left[P_{K}^{\leq 2}\right]-P_{K}^{\leq 2}\right)+\sum_{j \geq 2} \frac{1}{j !} \operatorname{ad}_{F}^{j}(P) .
\end{gathered}
$$

By (5.47), (5.48) and (5.35) we can apply Proposition 3.2 with $N_{0}, N_{0}^{\prime}, s^{\prime}, r^{\prime}, \theta^{\prime}, \mu^{\prime}, \delta \rightsquigarrow$ $N_{0}^{*}, N_{0}^{+}, s_{+}, r_{+}, \theta_{+}, \mu_{+}, \delta_{+}$. We get (recall $N_{0}^{*} \geq N_{0}$ )

$$
\begin{gathered}
\left\|\sum_{j \geq 2} \frac{1}{j !} \operatorname{ad}_{F}^{j}(P)\right\|_{s_{+}, r_{+}, N_{0}^{+}, \theta_{+}, \mu_{+}}^{T} \stackrel{(3.67),(3.35)}{\leftarrow}\left(\delta_{+}^{-1}\|F\|_{s, r, N_{0}^{*}, \theta, \mu}^{T}\right)^{2}\|P\|_{s, r, N_{0}, \theta, \mu}^{T} \\
\stackrel{\text { (5.53),(5.46) }}{\leftarrow} \delta_{+}^{-2} K^{2 \bar{\tau}} \bar{\varepsilon}^{2} \gamma \Theta
\end{gathered}
$$

and, similarly,

$$
\begin{gathered}
\left\|\sum_{j \geq 2} \frac{1}{j !} \operatorname{ad}_{F}^{j-1}\left(P_{K}^{\leq 2}\right)\right\|_{s_{+}, r_{+}, N_{0}^{+}, \theta_{+}, \mu_{+}}^{T}=\left\|\sum_{j \geq 1} \frac{1}{(j+1) !} \operatorname{ad}_{F}^{j}\left(P_{\bar{K}}^{\leq 2}\right)\right\|_{s_{+}, r_{+}, N_{0}^{+}, \theta_{+}, \mu_{+}}^{T} \\
\stackrel{\stackrel{(3.67)}{\lessdot} \delta_{+}^{-1}\|F\|_{s, r, N_{0}^{*}, \theta, \mu}^{T}\left\|P_{K}^{\leq 2}\right\|_{s, r, N_{0}, \theta, \mu}^{T}}{\stackrel{(5.53),(5.52)}{\lessdot} \delta_{+}^{-1} K^{\bar{\tau}} \gamma \bar{\varepsilon}^{2} .}
\end{gathered}
$$

Finally, by Proposition 3.1, applied with

$$
N_{0}, N_{1}, s_{1}, r_{1}, \theta_{1}, \mu_{1}, \delta \rightsquigarrow N_{0}^{*}, N_{0}^{+}, s_{+}, r_{+}, \theta_{+}, \mu_{+}, \delta_{+},
$$

we get

$$
\begin{aligned}
& \left\|\operatorname{ad}_{F}\left(P^{\leq 2}\right)\right\|_{s_{+}, r_{+}, N_{0}^{+}, \theta_{+}, \mu_{+}}^{T} \stackrel{(3.52)}{\leftarrow} \quad \delta_{+}^{-1}\|F\|_{s, r, N_{0}^{*}, \theta, \mu}^{T}\left\|P^{\leq 2}\right\|_{s, r, N_{0}, \theta, \mu}^{T} \\
& \stackrel{\text { (5.53),(5.52) }}{\leftarrow} \delta_{+}^{-1} K^{\bar{\tau}} \gamma \bar{\varepsilon}^{2} .
\end{aligned}
$$

The bounds (5.54), (5.55), (5.57), and $\Theta \leq 1$ (see (5.49)), prove the lemma. 
Lemma 5.5. - (5.51) holds.

Proof. - By Proposition 3.1 (applied with (5.56)) we have

$$
\begin{aligned}
& \left\|\operatorname{ad}_{F}\left(P^{\geq 3}\right)\right\|_{s_{+}, r_{+}, N_{0}^{+}, \theta_{+}, \mu_{+}}^{T} \quad \lessdot \quad \delta_{+}^{-1}\|F\|_{s, r, N_{0}^{*}, \theta, \mu}^{T}\left\|P^{\geq 3}\right\|_{s, r, N_{0}, \theta, \mu}^{T} \\
& \text { (5.53),(3.40),(5.46) } \delta_{+}^{-1} K^{\bar{\tau}} \gamma \bar{\varepsilon} \Theta,
\end{aligned}
$$

and (5.51) follows by (5.37), (3.40), (3.35), (5.46) (5.58), Lemma 5.4 and $\bar{\varepsilon} \leq 3 \Theta$ (which follows by (5.46) and (3.39)).

We now consider $P_{+}^{(h)}, h=0,1,2$. The term $\operatorname{ad}_{F} P^{\geq 3}$ in (5.37) does not contribute to $P_{+}^{(0)}$. On the contrary, its contribution to $P_{+}^{(1)}$ is

$$
\left\{F^{(0)}, P^{(3)}\right\}
$$

and to $P_{+}^{(2)}$ is

$$
\left\{F^{(1)}, P^{(3)}\right\}+\left\{F^{(0)}, P^{(4)}\right\}
$$

LEMmA 5.6. - $\left\|\left\{F^{(0)}, P^{(3)}\right\}\right\|_{s_{+}, r_{+}, N_{0}^{+}, \theta_{+}, \mu_{+}}^{T} \lessdot \delta_{+}^{-1} \gamma K^{\bar{\tau}} \varepsilon^{(0)} \Theta$ and

$$
\left\|\left\{F^{(1)}, P^{(3)}\right\}+\left\{F^{(0)}, P^{(4)}\right\}\right\|_{s_{+}, r_{+}, N_{0}^{+}, \theta_{+}, \mu_{+}}^{T} \lessdot \delta_{+}^{-1} K^{\bar{\tau}} \gamma\left(\varepsilon^{(0)}+\varepsilon^{(1)}\right) \Theta .
$$

Proof. - By (3.52) (applied with (5.56)), (5.53), (5.46) and (3.39).

The contribution of $P-P_{\bar{K}}^{\leq 2}$ in (5.37) to $P_{+}^{(h)}, h=0,1,2$, is $P_{>K}^{(h)}$.

LEMMA 5.7. $-\left\|P_{>K}^{(h)}\right\|_{s_{+}, r_{+}, N_{0}^{+}, \theta_{+}, \mu_{+}}^{T} \leq 2 e^{-K\left(s-s_{+}\right)} \gamma \varepsilon^{(h)}$

Proof. - By (3.43) and (5.46).

Proof of Proposition 5.2 Completed. Finally, (5.50) follows by (5.37), Lemmata 5.4, 5.6 (and (5.59)-(5.60)), Lemma 5.7 and $\Theta \leq 1$.

\subsection{KAM iteration}

Lemma 5.8. - Suppose that $\varepsilon_{i}^{(0)}, \varepsilon_{i}^{(1)}, \varepsilon_{i}^{(2)} \geq 0, i=0, \ldots, \nu$, satisfy

$$
\begin{aligned}
& \varepsilon_{i+1}^{(0)} \leq C_{*} \mathrm{~K}^{i} \bar{\varepsilon}_{i}^{2}+C_{*} \varepsilon_{i}^{(0)} e^{-K_{*} 2^{i}} \\
& \varepsilon_{i+1}^{(1)} \leq C_{*} \mathrm{~K}^{i}\left(\varepsilon_{i}^{(0)}+\bar{\varepsilon}_{i}^{2}\right)+C_{*} \varepsilon_{i}^{(1)} e^{-K_{*} 2^{i}} \\
& \varepsilon_{i+1}^{(2)} \leq C_{*} \mathrm{~K}^{i}\left(\varepsilon_{i}^{(0)}+\varepsilon_{i}^{(1)}+\bar{\varepsilon}_{i}^{2}\right)+C_{*} \varepsilon_{i}^{(2)} e^{-K_{*} 2^{i}}, \quad i=0, \ldots, \nu-1,
\end{aligned}
$$

where $\bar{\varepsilon}_{i}:=\varepsilon_{i}^{(0)}+\varepsilon_{i}^{(1)}+\varepsilon_{i}^{(2)}$, for some $\mathrm{K}, C_{*}, K_{*}>1$. Then there exist $\bar{\varepsilon}_{\star}<1, C_{\star}>0$, $\chi \in(1,2)$, depending only on $\mathrm{K}, C_{*}, K_{*}$ (and not on $\nu$ and satisfying $1 \leq C_{\star} e^{-K_{*}}$ ), such that, if

$$
\bar{\varepsilon}_{0} \leq \bar{\varepsilon}_{\star} \quad \Longrightarrow \quad \bar{\varepsilon}_{i} \leq C_{\star} \bar{\varepsilon}_{0} e^{-K_{*} \chi^{i}}, \forall i=0, \ldots, \nu
$$


Proof. - Iterating three times (5.61) we get

$$
\begin{aligned}
\bar{\varepsilon}_{j+3} & \leq c_{1} C_{*}^{c_{1}} K^{c_{1} j}\left(\varepsilon_{j+2}^{(0)}+\varepsilon_{j+2}^{(1)}+\bar{\varepsilon}_{j+2}^{2}+\bar{\varepsilon}_{j+2} e^{-K_{*} 2^{j+2}}\right) \\
& \leq c_{2} C_{*}^{c_{2}} K^{c_{2} j}\left(\varepsilon_{j+1}^{(0)}+\bar{\varepsilon}_{j+1}^{2}+\bar{\varepsilon}_{j+1}^{4}+\bar{\varepsilon}_{j+1} e^{-K_{*} 2^{j+1}}\right) \\
& \leq c_{3} C_{*}^{c_{3}} K^{c_{3} j}\left(\bar{\varepsilon}_{j}^{2}+\bar{\varepsilon}_{j}^{8}+\bar{\varepsilon}_{j} e^{-K_{*} 2^{j}}\right), \quad \forall 0 \leq j \leq \nu-3,
\end{aligned}
$$

for suitable constants $1<c_{1}<c_{2}<c_{3}$.

We first claim that (5.62) holds with $\chi:=6 / 5$ for all $i=3 j \leq \nu$. Setting $a_{j}:=\bar{\varepsilon}_{3 j}$, we prove that there exist $C_{\star}$ large and $\bar{\varepsilon}_{\star}$ small (as in the statement) such that if $a_{0} \leq \bar{\varepsilon}_{\star}$ then

$$
(\mathrm{S})_{j} \quad a_{j} \leq c_{4}^{j+1} a_{0} e^{-K_{*} \tilde{\chi}^{3 j}}, \quad \forall 0 \leq j \leq \nu / 3
$$

for a suitable $c_{4}=c_{4}\left(\mathrm{~K}, C_{*}, K_{*}\right) \geq 1$ large enough and $\tilde{\chi}<2^{1 / 3}$, e.g., $\tilde{\chi}:=5 / 4$. We proceed by induction. The statement $(\mathrm{S})_{0}$ is trivial. Now suppose $(\mathrm{S})_{j}$ holds true. Note that $a_{j} \leq 1$ taking $\bar{\varepsilon}_{\star} \leq \min _{j \geq 0} e^{K_{*} \tilde{\chi}^{3 j}} / c_{4}^{j+1}$. Then $(\mathrm{S})_{j+1}$ follows by

$$
\begin{aligned}
a_{j+1} & =\bar{\varepsilon}_{3 j+3} \stackrel{(5.63)}{\leq} c_{3} C_{*}^{c_{3}} \mathrm{~K}^{3 c_{3} j}\left(a_{j}^{2}+a_{j}^{8}+a_{j} e^{-K_{*} 2^{3 j}}\right) \stackrel{a_{j} \leq 1}{\leq} 2 c_{3} C_{*}^{c_{3}} \mathrm{~K}^{3 c_{3} j}\left(a_{j}^{2}+a_{j} e^{-K_{*} 2^{3 j}}\right) \\
& \stackrel{(\mathrm{S})_{j}}{\leq} 2 c_{3} C_{*}^{c_{3}} \mathrm{~K}^{3 c_{3} j}\left(\left(c_{4}^{j+1} a_{0} e^{-K_{*} \tilde{\chi}^{3 j}}\right)^{2}+\left(c_{4}^{j+1} a_{0} e^{-K_{*} \tilde{\chi}^{3 j}}\right) e^{-K_{*} 2^{3 j}}\right) \leq c_{4}^{j+2} a_{0} e^{-K_{*} \tilde{\chi}^{3 j+3}}
\end{aligned}
$$

since $4 c_{3} C_{*}^{c_{3}} \mathrm{~K}^{3 c_{3} j}\left(c_{4}^{j+1} a_{0} e^{-K_{*} \tilde{\chi}^{3 j}}\right) e^{-K_{*} 2^{3 j}} \leq c_{4}^{j+2} a_{0} e^{-K_{*} \tilde{\chi}^{3 j+3}}$ taking $c_{4}$ large enough (use $\tilde{\chi}<2$ ) and

$$
4 c_{3} C_{*}^{c_{3}} \mathrm{~K}^{3 c_{3} j}\left(c_{4}^{j+1} a_{0} e^{-K_{*} \tilde{\chi}^{3 j}}\right)^{2} \leq c_{4}^{j+2} a_{0} e^{-K_{*} \tilde{\chi}^{3 j+3}}
$$

taking $a_{0} \leq \bar{\varepsilon}_{\star}$ small enough. We have proved inductively (S) ${ }_{j}$. Then (5.62) for $i=3 j$ follows since $6 / 5=: \chi<\tilde{\chi}:=5 / 4$ and taking $C_{\star}$ large enough. The cases $i=3 j+1$ and $i=3 j+2$ follow analogously noting that $\bar{\varepsilon}_{1}, \bar{\varepsilon}_{2}$ can be made small by (5.61) taking $\bar{\varepsilon}_{\star}$ small.

For $\nu \in \mathbb{N}$, we define

$$
\begin{aligned}
s_{\nu+1} & :=s_{\nu}-s_{0} 2^{-\nu-2} \searrow \frac{s_{0}}{2}, & r_{\nu+1} & :=r_{\nu}-r_{0} 2^{-\nu-2} \searrow \frac{r_{0}}{2} \\
D_{\nu} & :=D\left(s_{\nu}, r_{\nu}\right) & K_{\nu} & :=K_{0} 4^{\nu} \\
N_{\nu} & :=N_{0} 2^{\nu \rho} \text { with } N_{0}:=\hat{c} \gamma^{-1 / 3} K_{0}^{\tau+1} & \rho & :=\max \left\{2(\tau+1), \frac{1}{L-b}, \frac{1}{1-L}\right\} \\
\mu_{\nu+1} & :=\mu_{\nu}-\mu_{0} 2^{-\nu-2} \searrow \frac{\mu_{0}}{2}, & \theta_{\nu+1} & :=\theta_{\nu}+\theta_{0} 2^{-\nu-2} \nearrow 3 \frac{\theta_{0}}{2} .
\end{aligned}
$$

We consider $H^{0}=\mathcal{N}_{0}+P_{0}: D_{0} \times \mathcal{O}_{*} \rightarrow \mathbb{C}$ with $\mathcal{N}_{0}:=e_{0}+\omega^{(0)}(\xi) \cdot y+\Omega^{(0)}(\xi) \cdot z \bar{z}$. We suppose that $\omega^{(0)}$ and $\Omega^{(0)}$ are defined on the whole $\mathbb{R}^{n}$ (using in case the Kirszbraun extension theorem), that $\Omega^{(0)}$ satisfies (4.2) and $\left|\omega^{(0)}\right|^{\text {lip }},\left|\Omega^{(0)}\right|_{\infty}^{\text {lip }} \leq M_{0}$ on $\mathbb{R}^{n}$. Let $\mathcal{O}_{0} \subseteq\left\{\xi \in \mathcal{O}_{*}: B_{\gamma / M_{0}}(\xi) \subset \mathcal{O}_{*}\right\}$ where $\mathcal{O}_{*}$ is defined in (5.1) and $B_{r}(\xi)$ denotes the open ball in $\mathbb{R}^{n}$ of center $\xi$ and radius $r>0$.

Lemma 5.9 (Iterative lemma). - Let $H^{0}$ be as above and let $\bar{\varepsilon}_{0}, \Theta_{0}$ be defined as in (5.46) for $P_{0}$. Then there are $K_{0}>0$ large enough, $\epsilon_{0}>0$ small enough, such that, if

$$
\bar{\varepsilon}_{0}, \Theta_{0} \leq \epsilon_{0}
$$

then 
$(\mathrm{S} 1)_{\nu} \forall 0 \leq i \leq \nu$, there exist $\omega^{(i)}, \Omega^{(i)}, a_{ \pm}^{(i)}$ defined for all $\xi \in \mathbb{R}^{n}$, satisfying

$$
\left|\omega^{(i)}-\omega^{(0)}\right|+\lambda\left|\omega^{(i)}-\omega^{(0)}\right|^{\text {lip }},\left|\Omega^{(i)}-\Omega^{(0)}\right|_{\infty}+\lambda\left|\Omega^{(i)}-\Omega^{(0)}\right|_{\infty}^{\operatorname{lip}_{\infty}} \leq C\left(1-2^{-i}\right) \gamma \bar{\varepsilon}_{0}
$$

$$
\left|a_{ \pm}^{(i)}\right| \leq C\left(1-2^{-i}\right) \gamma \bar{\varepsilon}_{0},\left|\omega^{(i)}\right|^{\text {lip }},\left|\Omega^{(i)}\right|_{\infty}^{\text {lip }} \leq\left(2-2^{-i}\right) M_{0} .
$$

There exists $H^{i}:=\mathcal{N}_{i}+P_{i}: D_{i} \times \mathcal{O}_{i} \rightarrow \mathbb{C}$ with $\mathcal{N}_{i}:=e_{i}+\omega^{(i)}(\xi) \cdot y+\Omega^{(i)}(\xi) \cdot z \bar{z}$ in normal form, where, for $i>0$,

$$
\begin{array}{r}
\mathcal{O}_{i}:=\left\{\xi \in \mathcal{O}_{i-1}:\left|\omega^{(i-1)}(\xi) \cdot k+\Omega^{(i-1)}(\xi) \cdot l\right| \geq\left(1-2^{-i}\right) \frac{2 \gamma}{1+|k|^{\tau}}, \forall(k, l) \in \mathbf{I},|k| \leq K_{i-1},\right. \\
\left.\left|\omega^{(i-1)}(\xi) \cdot k+p\right| \geq\left(1-2^{-i}\right) \frac{2 \gamma^{2 / 3}}{1+|k|^{\tau}}, \forall(k, p) \neq(0,0),|k| \leq K_{i-1}, p \in \mathbb{Z}\right\} .
\end{array}
$$

Moreover, $\forall 1 \leq i \leq \nu, H^{i}=H^{i-1} \circ \Phi^{i}$ where $\Phi^{i}: D_{i} \times \mathcal{O}_{i} \rightarrow D_{i-1}$ is a (Lipschitz) family (in $\xi \in \mathcal{O}_{i}$ ) of close-to-the-identity analytic symplectic maps. Setting, for $h=0,1,2$,

$$
\begin{aligned}
& \varepsilon_{i}^{(h)}:=\gamma^{-1} \max \left\{\left\|P_{i}^{(h)}\right\|_{s_{i}, r_{i}, N_{i}, \theta_{i}, \mu_{i}}^{T},\left|X_{P_{i}^{(h)}}\right|_{s_{i}, r_{i}}^{\lambda}\right\}, \quad \bar{\varepsilon}_{i}:=\sum_{h=0}^{2} \varepsilon_{i}^{(h)}, \\
& \Theta_{i}:=\gamma^{-1} \max \left\{\left\|P_{i}\right\|_{s_{i}, r_{i}, N_{i}, \theta_{i}, \mu_{i}}^{T},\left|X_{P_{i}}\right|_{s_{i}, r_{i}}^{\lambda}\right\}
\end{aligned}
$$

$\forall 1 \leq i \leq \nu$ and $\forall \xi \in \mathbb{R}^{n}$

$$
\left|\omega^{(i)}(\xi)-\omega^{(i-1)}(\xi)\right|,\left|\Omega^{(i)}(\xi)-\Omega^{(i-1)}(\xi)\right|_{\infty},\left|a_{ \pm}^{(i)}(\xi)-a_{ \pm}^{(i-1)}(\xi)\right| \leq 2 \gamma \bar{\varepsilon}_{i-1}
$$

$(\mathrm{S} 2)_{\nu} \forall 0 \leq i \leq \nu-1$, the $\varepsilon_{i}^{(0)}, \varepsilon_{i}^{(1)}, \varepsilon_{i}^{(2)}$ satisfy (5.61) with $\mathrm{K}=4^{2 \bar{\tau}+1}, \bar{\tau}:=2 \tau+n+1$, $C_{*}=4 K_{0}^{2 \bar{\tau}}, K_{*}=s_{0} K_{0} / 4$.

(S3) ${ }_{\nu} \forall 0 \leq i \leq \nu$, we have $\bar{\varepsilon}_{i} \leq C_{\star} \bar{\varepsilon}_{0} e^{-K_{*} \chi^{i}}$ and $\Theta_{i} \leq 2 \Theta_{0}$ (recall that $C_{\star} e^{-K_{*}} \geq 1$, see Lemma 5.8).

Proof. - The statement $(\mathrm{S} 1)_{0}$ follows by the hypotheses setting $a_{ \pm}^{(0)}(\xi):=0, \forall \xi \in \mathbb{R}^{n}$. $(\mathrm{S} 2)_{0}$ is empty. $(\mathrm{S} 3)_{0}$ is trivial. We then proceed by induction.

$(\mathrm{S} 1)_{\nu+1}$. We denote $\hat{\omega}^{(\nu)}:=\left.\nabla_{y}\left\langle P_{\nu}(\xi)\right\rangle\right|_{y=0, z=\bar{z}=0}$ and $\hat{\Omega}_{j}^{(\nu)}(\xi):=\partial_{z_{j} \bar{z}_{j} \mid y=0, z=\bar{z}=0}^{2}\left\langle P_{\nu}(\xi)\right\rangle$, see (5.36), for all $\xi \in \mathcal{O}_{\nu}$ if $\nu \geq 1$ and $\xi \in \mathcal{O}_{*}$ (see (5.1)) if $\nu=0$. By Lemma 5.2 and (5.69) there exist constants $\hat{a}_{ \pm}^{(\nu)}(\xi) \in \mathbb{R}$ such that

$\left|\hat{\omega}^{(\nu)}(\xi)\right|,\left|\hat{\Omega}^{(\nu)}(\xi)\right|_{\infty},\left|\hat{a}_{ \pm}^{(\nu)}(\xi)\right| \leq 2 \gamma \bar{\varepsilon}_{\nu}, \quad\left|\hat{\Omega}_{j}^{(\nu)}(\xi)-\hat{a}_{\mathbf{s}(j)}^{(\nu)}(\xi)\right| \leq 40 \gamma \frac{\bar{\varepsilon}_{\nu}}{|j|}, \forall|j| \geq 6\left(N_{\nu}+1\right)$, uniformly in $\xi \in \mathcal{O}_{\nu}$ (resp. $\mathcal{O}_{*}$ if $\nu=0$ ), and

$$
\left|\hat{\omega}^{(\nu)}\right|^{\text {lip }},\left|\hat{\Omega}^{(\nu)}\right|_{\infty}^{\text {lip }} \leq C \bar{\varepsilon}_{\nu} .
$$

Let

$$
\eta_{0}:=\lambda=\gamma / M_{0}, \quad \eta_{\nu}:=\gamma /\left(2^{\nu+3} M_{0} K_{\nu-1}^{\tau+1}\right), \nu \geq 1
$$


We claim that, for $\nu \geq 1$, the $\eta_{\nu}$-neighborhood of $\mathcal{O}_{\nu+1}$

$$
\tilde{\mathcal{O}}_{\nu+1}:=\bigcup_{\xi \in \mathcal{O}_{\nu+1}}\left\{\tilde{\xi} \in \mathbb{R}^{n}: \tilde{\xi}=\xi+\hat{\xi},|\hat{\xi}|<\eta_{\nu}\right\} \subseteq \mathcal{O}_{\nu}
$$

Note that the definitions of $\mathcal{O}_{0}, \mathcal{O}_{1}$ in (5.68), and (5.73) imply $\tilde{\mathcal{O}}_{1} \subset \mathcal{O}_{*}$. Recalling (5.68), we have to prove that for $\nu \geq 1$, for every $\tilde{\xi}=\xi+\hat{\xi}, \xi \in \mathcal{O}_{\nu+1},|\hat{\xi}| \leq \eta_{\nu}$, we have

$$
\left|\omega^{(\nu-1)}(\tilde{\xi}) \cdot k+\Omega^{(\nu-1)}(\tilde{\xi}) \cdot l\right| \geq\left(1-2^{-\nu}\right) \frac{2 \gamma}{1+|k|^{\tau}}, \quad \forall(k, l) \in \mathbf{I},|k| \leq K_{\nu-1},
$$

and the analogous estimate for $\left|\omega^{(\nu-1)}(\tilde{\xi}) \cdot k+p\right|$. By the expression (5.77) (at the previous step) for $\omega^{(\nu)}, \Omega^{(\nu)}$, and since $\chi_{\nu-1} \in[0,1]$, we get

$$
\begin{aligned}
& \left|\omega^{(\nu-1)}(\tilde{\xi}) \cdot k+\Omega^{(\nu-1)}(\tilde{\xi}) \cdot l\right| \geq\left|\omega^{(\nu)}(\tilde{\xi}) \cdot k+\Omega^{(\nu)}(\tilde{\xi}) \cdot l\right| \\
& \quad-\left|\chi_{\nu-1}(\tilde{\xi})\right|\left|\hat{\omega}^{(\nu-1)}(\tilde{\xi}) \cdot k+\hat{\Omega}^{(\nu-1)}(\tilde{\xi}) \cdot l\right| \\
& \quad \stackrel{(5.71)}{\geq}\left|\omega^{(\nu)}(\xi) \cdot k+\Omega^{(\nu)}(\xi) \cdot l\right|-\left|\left(\omega^{(\nu)}(\tilde{\xi})-\omega^{(\nu)}(\xi)\right) \cdot k+\left(\Omega^{(\nu)}(\tilde{\xi})-\Omega^{(\nu)}(\xi)\right) \cdot l\right| \\
& \quad-2 \gamma \bar{\varepsilon}_{\nu-1}\left(K_{\nu-1}+2\right) \\
& \quad \stackrel{\xi \in \mathcal{O}_{\nu+1},(5.68),(S 1)_{\nu}}{\geq}\left(1-2^{-\nu-1}\right) \frac{2 \gamma}{1+|k|^{\tau}}-\left(K_{\nu-1}+2\right) 2 M_{0} \eta_{\nu}-2 \gamma \bar{\varepsilon}_{\nu-1}\left(K_{\nu-1}+2\right) \\
& \quad{ }^{(5.73),(S 3)_{\nu}}\left(1-2^{-\nu}\right) \frac{2 \gamma}{1+|k|^{\tau}}
\end{aligned}
$$

taking $\epsilon_{0}$ small enough, and (5.75) follows. The estimate for $\left|\omega^{(\nu-1)}(\tilde{\xi}) \cdot k+p\right|$ follows similarly.

We define a smooth cut-off function $\chi_{\nu}: \mathbb{R}^{n} \rightarrow[0,1]$ which takes value 1 on $\mathcal{O}_{\nu+1}$ and value 0 outside $\tilde{\mathcal{O}}_{\nu+1}$. Thanks to (5.74) and recalling (5.73) we can construct $\chi_{\nu}, \nu \geq 0$, in such a way that

$$
\left|\chi_{\nu}\right|^{\text {lip }} \gamma^{-1} M_{0} 2^{\nu} K_{\nu-1}^{\tau+1}
$$

where $K_{-1}:=1$. We extend $\hat{\omega}^{(\nu)}, \hat{\Omega}^{(\nu)}, \hat{a}_{ \pm}^{(\nu)}$ to zero outside $\mathcal{O}_{\nu}$ for $\nu \geq 1$ and, for $\nu=0$ outside $\mathcal{O}_{\star}$. Then we define on the whole $\mathbb{R}^{n}$

$$
\omega^{(\nu+1)}:=\omega^{(\nu)}+\chi_{\nu} \hat{\omega}^{(\nu)}, \quad \Omega^{(\nu+1)}:=\Omega^{(\nu)}+\chi_{\nu} \hat{\Omega}^{(\nu)}, \quad a_{ \pm}^{(\nu+1)}:=a_{ \pm}^{(\nu)}+\chi_{\nu} \hat{a}_{ \pm}^{(\nu)} .
$$

By (5.76), (5.72), (5.71), we get

$$
\left|\omega^{(\nu+1)}-\omega^{(\nu)}\right|^{\text {lip }} \leq\left|\chi_{\nu}\right|^{\text {lip }}\left|\hat{\omega}^{(\nu)}\right|+\left|\chi_{\nu}\right|\left|\hat{\omega}^{(\nu)}\right|^{\text {lip }} \leq C K_{\nu-1}^{\tau+1} M_{0} \bar{\varepsilon}_{\nu}+C \bar{\varepsilon}_{\nu} \leq 2^{-\nu-1} M_{0}
$$

by $(\mathrm{S} 3)_{\nu}$ and $\bar{\varepsilon}_{0}$ small enough. Similarly for $\left|\Omega^{(\nu+1)}-\Omega^{(\nu)}\right|_{\infty}^{\text {lip }}$. Recalling also (5.71), we get (5.66) and (5.67) with $i=\nu+1$. Moreover (5.71)-(5.77) imply (5.70) for $i=\nu+1$ and $\forall|j|>6\left(N_{\nu}+1\right)$.

We wish to apply the KAM step Proposition 5.2 with $\mathcal{N}=\mathcal{N}_{\nu}, P=P_{\nu}, N_{0}=N_{\nu}$, $\theta=\theta_{\nu} \ldots$ and $N_{0}^{+}=N_{\nu+1}, \theta_{+}=\theta_{\nu+1}, \ldots$ Our definitions in (5.64) (and $\tau>1 / b$ ) imply that the conditions ${ }^{(2)}$ (5.47)-(5.48) are satisfied, for all $\nu \in \mathbb{N}$, taking $K_{0}$ large enough. Moreover,

(2) For example the first inequality in (5.47) reads $N_{\nu+1} \geq \max \left\{N_{\nu}, \hat{c} \gamma^{-1 / 3} K_{\nu}^{\tau+1}, \bar{N}\right\}$. 
since

$$
\delta^{+}=\delta_{\nu+1}:=\min \left\{1-\frac{s_{\nu+1}}{s_{\nu}}, 1-\frac{r_{\nu+1}}{r_{\nu}}\right\} \quad \text { so that } \quad 2^{-\nu-2} \leq \delta_{\nu+1} \leq 2^{-\nu-1},
$$

and $(S 3)_{\nu}$ the condition (5.49) is satisfied, for $\bar{\varepsilon}_{0} \leq \epsilon_{0}$ small enough, $\forall \nu \in \mathbb{N}$. By (5.70), the condition (5.11) holds for $|j| \geq \theta_{\nu} N_{\nu}$, and (5.14) and (5.19) hold for all $\xi \in \mathcal{O}_{\nu+1}$ (it is the definition of $\mathcal{O}_{\nu+1}$, see (5.68)). Hence Proposition 5.2 applies. For all $\xi \in \mathcal{O}_{\nu+1}$ the Hamiltonian flow $\Phi^{\nu+1}:=\Phi_{F_{\nu}}^{1}: D_{\nu+1} \times \mathcal{O}_{\nu+1} \rightarrow D_{\nu}$ and we define

$$
H^{\nu+1}:=H^{\nu} \circ \Phi^{\nu+1}=e^{\operatorname{ad}_{\mathrm{F}_{\nu}}} H^{\nu}=\mathcal{N}_{\nu+1}+P_{\nu+1}: D_{\nu+1} \times \mathcal{O}_{\nu+1} \rightarrow \mathbb{C} .
$$

$(\mathrm{S} 2)_{\nu+1}$ follows by (5.50) and (5.64).

$(\mathrm{S} 3)_{\nu+1}$. By $(S 2)_{\nu}$ we can apply Lemma 5.8 and (5.62) implies $\bar{\varepsilon}_{\nu+1} \leq C_{\star} \bar{\varepsilon}_{0} e^{-K_{*} \chi^{\nu+1}}$. Moreover, for $\epsilon_{0}$ small enough, $\Theta_{\nu+1} \stackrel{(5.51)}{\leq} \Theta_{0} \Pi_{i=0}^{\nu}\left(1+C \delta_{i+1}^{-2} K_{i}^{2 \bar{\tau}} \bar{\varepsilon}_{i}\right) \stackrel{(5.78),(S 3)_{\nu}}{\leq} 2 \Theta_{0}$.

Proof of the KAM Theorem 4.1 completed. - We apply the iterative Lemma 5.9 to the Hamiltonian $H^{0}$ in (5.6) where $\omega^{(0)}=\omega$ and $\Omega^{(0)}=\Omega$ are defined in (4.1). We choose

$$
\mathcal{O}_{0}:=\left\{\xi \in \mathcal{O}: \quad|\omega(\xi) \cdot k| \geq \frac{2 \gamma^{2 / 3}}{1+|k|^{n}}, \forall 0<|k|<\gamma^{-1 /(7 n)}\right\}
$$

so that $\mathcal{O}_{0} \subseteq\left\{\xi \in \mathcal{O}_{*}: B_{\gamma / M_{0}}(\xi) \subset \mathcal{O}_{*}\right\}$, see (5.1) and (4.3). The smallness assumption (5.65) holds by (5.8)-(5.9) (use also Lemma 3.4) and $\varepsilon$ small enough. Then the iterative Lemma 5.9 applies. Let us define

$$
\omega^{\infty}:=\lim _{\nu \rightarrow \infty} \omega^{(\nu)}, \quad \Omega^{\infty}:=\lim _{\nu \rightarrow \infty} \Omega^{(\nu)}, \quad a_{ \pm}^{\infty}:=\lim _{\nu \rightarrow \infty} a_{ \pm}^{(\nu)} .
$$

It could happen that $\mathcal{O}_{\nu_{0}}=\varnothing$ for some $\nu_{0}$. In such a case $\mathcal{O}_{\infty}=\varnothing$ and the iterative process stops after finitely many steps. However, we can always set $\omega^{(\nu)}:=\omega^{\left(\nu_{0}\right)}, \Omega^{(\nu)}:=\Omega^{\left(\nu_{0}\right)}$, $a_{ \pm}^{(\nu)}:=a_{ \pm}^{\left(\nu_{0}\right)}, \forall \nu \geq \nu_{0}$, and $\omega^{\infty}, \Omega^{\infty}, a_{ \pm}^{\infty}$ are always well defined.

The bounds (4.8) follow by (5.66) (with a different constant $C$ ). We now prove (4.9). We consider the case $j>0$. For all $\forall \nu \geq 0, j \geq 6\left(N_{\nu}+1\right)$, we have (recall that $a_{+}^{(0)}=0$ )

$$
\begin{aligned}
\left|\Omega_{j}^{\infty}-\Omega_{j}^{(0)}-a_{+}^{\infty}\right| \leq & \sum_{0 \leq i \leq \nu}\left|\Omega_{j}^{(i+1)}-a_{+}^{(i+1)}-\Omega_{j}^{(i)}+a_{+}^{(i)}\right| \\
& +\sum_{i>\nu}\left|\Omega_{j}^{(i+1)}-\Omega_{j}^{(i)}\right|+\left|a_{+}^{(i+1)}-a_{+}^{(i)}\right| \\
& \stackrel{(5.70)}{\leq} 40 \gamma \sum_{0 \leq i \leq \nu} \frac{\bar{\varepsilon}_{i}}{j}+4 \gamma \sum_{i>\nu} \bar{\varepsilon}_{i} \stackrel{(\mathrm{S} 3)_{\nu}}{\leftarrow} \frac{\bar{\varepsilon}_{0} \gamma}{j}+\gamma \sum_{i>\nu} \bar{\varepsilon}_{i} .
\end{aligned}
$$

Therefore, $\forall \nu \geq 0,6\left(N_{\nu}+1\right) \leq j<6\left(N_{\nu+1}+1\right)$,

$$
\left|\Omega_{j}^{\infty}-\Omega_{j}^{(0)}-a_{+}^{\infty}\right| \lessdot \frac{\bar{\varepsilon}_{0} \gamma}{j}+\gamma \frac{N_{\nu+1}}{j} \sum_{i>\nu} \bar{\varepsilon}_{i} \stackrel{(5.64)}{\lessdot} \frac{\bar{\varepsilon}_{0} \gamma}{j}+\frac{\gamma}{j} \gamma^{-1 / 3} K_{0}^{\tau+1} 2^{\rho(\nu+1)} \sum_{i>\nu} \bar{\varepsilon}_{i}
$$

and (4.9) follows by $(S 3)_{\nu}$.

The symplectic transformation $\Phi$ in (4.10) is defined by

$$
\Phi:=\lim _{\nu \rightarrow \infty} \Phi_{00} \circ \Phi_{0} \circ \Phi_{1} \circ \cdots \circ \Phi^{\nu}
$$

with $\Phi_{00}$ defined in (5.4). We now verify that $\Phi$ is defined on $\mathcal{O}_{\infty}$, see (4.11). 
Lemma 5.10. $-\mathcal{O}_{\infty} \subset \cap_{i} \mathcal{O}_{i}$ (defined in (5.68)).

Proof. - We have $\mathcal{O}_{\infty} \subseteq \mathcal{O}_{0}$ by (4.11) and (5.79). For $i \geq 1$, if $\xi \in \mathcal{O}_{\infty}$ then, for all $|k| \leq K_{i},|l| \leq 2$,

$$
\begin{aligned}
& \left|\omega^{(i)}(\xi) \cdot k+\Omega^{(i)}(\xi) \cdot l\right| \\
& \geq\left|\omega^{\infty}(\xi) \cdot k+\Omega^{\infty}(\xi) \cdot l\right|-|k| \sum_{n \geq i}\left|\omega^{(n+1)}(\xi)-\omega^{(n)}(\xi)\right|-2 \sum_{n \geq i}\left|\Omega^{(n+1)}(\xi)-\Omega^{(n)}(\xi)\right|_{\infty} \\
& \stackrel{(4.11),(5.70)}{\geq} \frac{2 \gamma}{1+|k|^{\tau}}-K_{i} 2 \gamma \sum_{n \geq i} \bar{\varepsilon}_{n}-4 \gamma \sum_{n \geq i} \bar{\varepsilon}_{n} \geq\left(1-2^{-i}\right) \frac{2 \gamma}{1+|k|^{\tau}}
\end{aligned}
$$

by the definition of $K_{i}$ in (5.64), $(S 3)_{\nu}$ and $\varepsilon$ small enough. The other estimate is analogous.

Finally $P_{\leq 2}^{\infty}=0$ (see (4.12)) follows by $\bar{\varepsilon}_{i} \rightarrow 0$ as $i \rightarrow \infty$. This concludes the proof of Theorem 4.1.

\section{Measure estimates: proof of Theorem 4.2}

We have to estimate the measure of

$$
\mathcal{O} \backslash \mathcal{O}_{\infty}=\bigcup_{(k, l) \in \Lambda_{0} \cup \Lambda_{1} \cup \Lambda_{2}^{+} \cup \Lambda_{2}^{-}} \mathcal{R}_{k l}(\gamma) \bigcup_{(k, p) \in \mathbb{Z}^{n+1} \backslash\{0\}} \tilde{\mathcal{R}}_{k p}\left(\gamma^{2 / 3}\right) \bigcup\left(\mathcal{O} \backslash \mathcal{O}_{0}\right)
$$

where

$$
\begin{aligned}
& \mathcal{R}_{k l}(\gamma):=\mathcal{R}_{k l}^{\tau}(\gamma):=\left\{\xi \in \mathcal{O}:\left|\omega^{\infty}(\xi) \cdot k+\Omega^{\infty}(\xi) \cdot l\right|<\frac{2 \gamma}{1+|k|^{\tau}}\right\} \\
& \tilde{\mathcal{R}}_{k p}\left(\gamma^{2 / 3}\right):=\tilde{\mathcal{R}}_{k p}^{\tau}\left(\gamma^{2 / 3}\right):=\left\{\xi \in \mathcal{O}:\left|\omega^{\infty}(\xi) \cdot k+p\right|<\frac{2 \gamma^{2 / 3}}{1+|k|^{\tau}}\right\}
\end{aligned}
$$

and

$$
\begin{aligned}
\Lambda_{h}:=\{(k, l) \in \mathbf{I}(\operatorname{see}(4.5)),|l|=h\}, & h=0,1,2, \quad \Lambda_{2}=\Lambda_{2}^{+} \cup \Lambda_{2}^{-}, \\
\Lambda_{2}^{+}:=\left\{(k, l) \in \Lambda_{2}, l= \pm\left(e_{i}+e_{j}\right)\right\}, & \Lambda_{2}^{-}:=\left\{(k, l) \in \Lambda_{2}, l=e_{i}-e_{j}\right\} .
\end{aligned}
$$

We first consider the most difficult case $\Lambda_{2}^{-}$. Setting $\mathcal{R}_{k, i, j}(\gamma):=\mathcal{R}_{k, e_{i}-e_{j}}(\gamma)$ we show that

$$
\left|\bigcup_{(k, l) \in \Lambda_{2}^{-}} \mathcal{R}_{k, l}(\gamma)\right|=\left|\bigcup_{(k, i, j) \in \mathrm{I}} \mathcal{R}_{k, i, j}(\gamma)\right| \lessdot \gamma^{2 / 3} \rho^{n-1}
$$

where

$$
\mathrm{I}:=\left\{(k, i, j) \in \mathbb{Z}^{n} \times(\mathbb{Z} \backslash \mathcal{I})^{2}:(k, i, j) \neq(0, i, i), \mathrm{j} \cdot k+i-j=0\right\} .
$$

Note that the indices in I satisfy

$$
\| i|-| j|| \leq \kappa|k| \text { and } k \neq 0 .
$$

Since the matrix $A$ in (4.13) is invertible, the bound (4.8) implies, for $\varepsilon$ small enough, that

$$
\omega^{\infty}: \mathcal{O} \rightarrow \omega^{\infty}(\mathcal{O}) \text { is invertible and }\left|\left(\omega^{\infty}\right)^{-1}\right|^{\text {lip }} \leq 2\left\|A^{-1}\right\| .
$$


Lemma 6.1. - For $(k, i, j) \in \mathrm{I}, \eta \in(0,1)$, we have

$$
\left|\mathcal{R}_{k, i, j}^{\tau}(\eta)\right| \lessdot \frac{\eta \rho^{n-1}}{1+|k|^{\tau+1}} .
$$

Proof. - By (4.8) and (4.13)

$$
\omega^{\infty}(\xi) \cdot k+\Omega_{i}^{\infty}(\xi)-\Omega_{j}^{\infty}(\xi)=\omega^{\infty}(\xi) \cdot k+\sqrt{i^{2}+\mathrm{m}}-\sqrt{j^{2}+\mathrm{m}}+r_{i, j}(\xi)
$$

where

$$
\left|r_{i, j}(\xi)\right|=O(\varepsilon \gamma),\left|r_{i, j}\right|^{\text {lip }}=O(\varepsilon) .
$$

We introduce the final frequencies $\zeta:=\omega^{\infty}(\xi)$ as parameters (see (6.8)), and we consider

$$
f_{k, i, j}(\zeta):=\zeta \cdot k+\sqrt{i^{2}+\mathrm{m}}-\sqrt{j^{2}+\mathrm{m}}+\tilde{r}_{i, j}(\zeta)
$$

where also $\tilde{r}_{i, j}:=r_{i, j} \circ\left(\omega^{\infty}\right)^{-1}$ satisfies (6.10). In the direction $\zeta=s k|k|^{-1}+w, w \cdot k=0$, the function $\tilde{f}_{k, i, j}(s):=f_{k, i, j}\left(s k|k|^{-1}+w\right)$ satisfies

$$
\tilde{f}_{k, i, j}\left(s_{2}\right)-\tilde{f}_{k, i, j}\left(s_{1}\right) \stackrel{(6.10)}{\geq}\left(s_{2}-s_{1}\right)(|k|-C \varepsilon) \geq\left(s_{2}-s_{1}\right)|k| / 2 .
$$

Since $|k| \geq 1$ (recall (6.7)), by Fubini theorem,

$$
\left|\left\{\zeta \in \omega^{\infty}(\mathcal{O}):\left|f_{k, i, j}(\zeta)\right| \leq \frac{2 \eta}{1+|k|^{\tau}}\right\}\right| \lessdot \frac{\eta \rho^{n-1}}{1+|k|^{\tau+1}} .
$$

By (6.8) the bound (6.9) follows.

We split

(6.11) $\mathrm{I}=\mathrm{I}_{>} \cup \mathrm{I}_{<} \quad$ where $\quad \mathrm{I}_{>}:=\left\{(k, i, j) \in \mathrm{I}: \min \{|i|,|j|\}>C_{\sharp} \gamma^{-1 / 3}\left(1+|k|^{\tau_{0}}\right)\right\}$ where $C_{\sharp}>C_{\star}$ in $(4.9)$ and $\tau_{0}:=n+1$. We set $\mathrm{I}_{<}:=\mathrm{I} \backslash \mathrm{I}_{>}$.

Lemma 6.2. - For all $(k, i, j) \in \mathrm{I}_{>}$we have

$$
\mathcal{R}_{k, i, j}^{\tau_{0}}\left(\gamma^{2 / 3}\right) \subset \mathcal{R}_{k, i_{0}, j_{0}}^{\tau_{0}}\left(2 \gamma^{2 / 3}\right)
$$

(see (6.2)), $i_{0}, j_{0} \in \mathbb{Z} \backslash \mathcal{I}$ satisfy

$$
\mathbf{s}\left(i_{0}\right)=\mathbf{s}(i), \mathbf{s}\left(j_{0}\right)=\mathbf{s}(j),\left|i_{0}\right|-\left|j_{0}\right|=|i|-|j|
$$

and

$$
\min \left\{\left|i_{0}\right|,\left|j_{0}\right|\right\}=\left[C_{\sharp} \gamma^{-1 / 3}\left(1+|k|^{\tau_{0}}\right)\right] .
$$

Proof. - Since $|j| \geq \gamma^{-1 / 3} C_{\star}$, by (4.9) and (4.13) we have the frequency asymptotic

$$
\Omega_{j}^{\infty}(\xi)=|j|+\frac{\mathrm{m}}{2|j|}+\vec{a} \cdot \xi+a_{\mathbf{s}(j)}^{\infty}(\xi)+O\left(\frac{\mathrm{m}^{2}}{|j|^{3}}\right)+O\left(\varepsilon \frac{\gamma^{2 / 3}}{|j|}\right) .
$$


By (6.7) we have $\| i|-| j||=|| i_{0}|-| j_{0}|| \leq C|k|,|k| \geq 1$. If $\xi \in \mathcal{O} \backslash \mathcal{R}_{k, i_{0}, j_{0}}^{\tau_{0}}\left(2 \gamma^{2 / 3}\right)$, since $|i|,|j| \geq \mu_{0}:=\min \left\{\left|i_{0}\right|,\left|j_{0}\right|\right\}$ (recall (6.11) and (6.14)), we have

$$
\begin{aligned}
\left|\omega^{\infty}(\xi) \cdot k+\Omega_{i}^{\infty}(\xi)-\Omega_{j}^{\infty}(\xi)\right| \geq & \left|\omega^{\infty}(\xi) \cdot k+\Omega_{i_{0}}^{\infty}(\xi)-\Omega_{j_{0}}^{\infty}(\xi)\right| \\
& -\left|\Omega_{i}^{\infty}(\xi)-\Omega_{i_{0}}^{\infty}(\xi)-\Omega_{j}^{\infty}(\xi)+\Omega_{j_{0}}^{\infty}(\xi)\right| \\
\stackrel{(6.15)}{\geq} & \frac{4 \gamma^{2 / 3}}{1+|k|^{\tau_{0}}}-|| i|-| i_{0}|-| j|+| j_{0}|| \\
& -\left|a_{\mathbf{s}(i)}^{\infty}-a_{\mathbf{s}\left(i_{0}\right)}^{\infty}-a_{\mathbf{s}(j)}^{\infty}+a_{\mathbf{s}\left(j_{0}\right)}^{\infty}\right| \\
& -C \varepsilon \frac{\gamma^{2 / 3}}{\mu_{0}}-C \frac{\mathrm{m}^{2}}{\mu_{0}^{3}}-\frac{\mathrm{m}}{2} \frac{|| i|-| j||}{|i||j|}-\frac{\mathrm{m}}{2} \frac{|| i_{0}|-| j_{0}||}{\left|i_{0}\right|\left|j_{0}\right|} \\
(\text { (6.13) } & \frac{4 \gamma^{2 / 3}}{1+|k|^{\tau_{0}}}-C \varepsilon \frac{\gamma^{2 / 3}}{\mu_{0}}-C \frac{|k|}{\mu_{0}^{2}} \stackrel{(6.14)}{\geq} \frac{2 \gamma^{2 / 3}}{1+|k|^{\tau_{0}}}
\end{aligned}
$$

taking $C_{\sharp}$ in (6.14) large enough. Therefore $\xi \in \mathcal{O} \backslash \mathcal{R}_{k, i, j}^{\tau_{0}}\left(\gamma^{2 / 3}\right)$ proving (6.12).

As a corollary we deduce:

Lemma 6.3. - $\left|\bigcup_{(k, i, j) \in \mathrm{I}>} \mathcal{R}_{k, i, j}^{\tau}(\gamma)\right| \lessdot \gamma^{2 / 3} \rho^{n-1}$.

Proof. - Since $0<\gamma \leq 1$ and $\tau \geq \tau_{0}$ (see (4.15)), we have (see (6.2)) $\mathcal{R}_{k, i, j}^{\tau}(\gamma) \subset$ $\mathcal{R}_{k, i, j}^{\tau_{0}}\left(\gamma^{2 / 3}\right)$. Then Lemma 6.2 and (6.9) imply that, for each $k \in \mathbb{Z}^{n}, p \in \mathbb{Z}$ fixed

$$
\left|\bigcup_{(k, i, j) \in \mathrm{I}_{>},|i|-|j|=p} \mathcal{R}_{k, i, j}^{\tau}(\gamma)\right| \lessdot \frac{\gamma^{2 / 3} \rho^{n-1}}{1+|k|^{\tau_{0}+1}} .
$$

Therefore

$$
\left|\bigcup_{(k, i, j) \in \mathrm{I}>} \mathcal{R}_{k, i, j}^{\tau}(\gamma)\right| \lessdot \sum_{k,|p| \leq C|k|} \frac{\gamma^{2 / 3} \rho^{n-1}}{1+|k|^{\tau_{0}+1}} \lessdot \sum_{k} \frac{\gamma^{2 / 3} \rho^{n-1}}{1+|k|^{\tau_{0}}}
$$

proving the lemma.

Lemma 6.4. - $\left|\bigcup_{(k, i, j) \in \mathrm{I}_{<}} \mathcal{R}_{k, i, j}^{\tau}(\gamma)\right| \lessdot \gamma^{2 / 3} \rho^{n-1}$.

Proof. - For all $(k, i, j) \in \mathrm{I}_{<}$such that $\mathcal{R}_{k, i, j}^{\tau}(\gamma) \neq \varnothing$ we have (see (6.6)) $\min \{|i|,|j|\}<C_{\sharp} \gamma^{-1 / 3}\left(1+|k|^{\tau_{0}}\right), j-i=k \cdot j \Longrightarrow \max \{|i|,|j|\}<C^{\prime} \gamma^{-1 / 3}\left(1+|k|^{\tau_{0}}\right)$.

Therefore, using also Lemma 6.1 and (6.7)

$$
\left|\bigcup_{(k, i, j) \in \mathrm{I}_{<}} \mathcal{R}_{k, i, j}^{\tau}(\gamma)\right| \lessdot \sum_{k} \sum_{\substack{|i| \leq C^{\prime} \gamma^{-1 / 3}\left(1+|k|^{\tau}\right) \\ j=i+k \cdot j}} \frac{\gamma \rho^{n-1}}{1+|k|^{\tau+1}} \lessdot \sum_{k} \frac{\gamma^{2 / 3} \rho^{n-1}}{1+|k|^{\tau-\tau_{0}+1}}
$$

which, by (4.15), gives the lemma.

Lemmata 6.3, 6.4 imply (6.5). This concludes the case $(k, l) \in \Lambda_{2}^{-}$. Let consider the other cases. The analogue of Lemma 6.1 is

Lemma 6.5. - For $(k, l) \in \Lambda_{0} \cup \Lambda_{1} \cup \Lambda_{2}^{+}, \eta \in(0, \sqrt{\mathrm{m}} / 2)$, we have

$$
\left|\mathcal{R}_{k l}(\eta)\right| \lessdot \frac{\eta \rho^{n-1}}{1+|k|^{\tau}} .
$$


Proof. - We consider only the case $(k, l) \in \Lambda_{2}^{+}, l=e_{i}+e_{j}$. By (4.8) and (4.13) $f_{k, i, j}(\xi):=\omega^{\infty}(\xi) \cdot k+\Omega_{i}^{\infty}(\xi)+\Omega_{j}^{\infty}(\xi)=\omega^{\infty}(\xi) \cdot k+\sqrt{i^{2}+\mathrm{m}}+\sqrt{j^{2}+\mathrm{m}}+2 \vec{a} \cdot \xi+r_{i, j}(\xi)$ where $\left|r_{i, j}(\xi)\right|=O(\varepsilon \gamma),\left|r_{i, j}\right|^{\text {lip }}=O(\varepsilon)$. Changing variables $\zeta:=\omega_{\infty}(\xi)$ we find

$$
f_{k, i, j}(\zeta):=\zeta \cdot k+\sqrt{i^{2}+\mathrm{m}}+\sqrt{j^{2}+\mathrm{m}}+2 \vec{a} \cdot A^{-1}(\zeta-\bar{\omega})+\tilde{r}_{i, j}(\zeta)
$$

where also

$$
\tilde{r}_{i, j}(\zeta)=O(\varepsilon \gamma),\left|\tilde{r}_{i, j}\right|^{\text {lip }}=O(\varepsilon) .
$$

If $k=\vec{a}=0$ then the function in (6.17) is bigger than $\sqrt{\mathrm{m}}$ and $\mathcal{R}_{0 l}(\eta)=\varnothing$, for $0 \leq \eta \leq \sqrt{\mathrm{m}} / 2$. Otherwise, by (4.14), the vector

$$
\text { (6.19) } \quad \tilde{a}:=A^{T} k+2 \vec{a}=A^{T}\left(k+2\left(A^{-1}\right)^{T} \vec{a}\right) \quad \text { satisfies } \quad|\tilde{a}| \geq c=c(A, \vec{a})>0, \forall k \neq 0 \text {. }
$$

The function $\tilde{f}_{k, i, j}(s):=f_{k, i, j}\left(s \tilde{a}|\tilde{a}|^{-1}+w\right), \tilde{a} \cdot w=0$, satisfies $\tilde{f}_{k, i, j}\left(s_{2}\right)-\tilde{f}_{k, i, j}\left(s_{1}\right) \geq$ $\left(s_{2}-s_{1}\right)(|\tilde{a}|-C \varepsilon) \geq\left(s_{2}-s_{1}\right)|\tilde{a}| / 2$ by (6.18). Then (6.16) follows by (6.19) and Fubini theorem.

By Lemma 6.5, (6.2), (6.3), (5.79) and standard arguments (as above)

$$
\left|\bigcup_{(k, l) \in \Lambda_{0} \cup \Lambda_{1} \cup \Lambda_{2}^{+}} \mathcal{R}_{k l}(\gamma)\right| \lessdot \gamma \rho^{n-1},\left|\bigcup_{(k, p) \in \mathbb{Z}^{n+1} \backslash\{0\}} \tilde{\mathcal{R}}_{k p}\left(\gamma^{2 / 3}\right)\right|,\left|\mathcal{O} \backslash \mathcal{O}_{0}\right| \lessdot \gamma^{2 / 3} \rho^{n-1} .
$$

Finally (6.1), (6.5), (6.20) imply (4.16).

\section{Application to DNLW}

For $\vec{\jmath}=\left(j_{1}, \ldots, j_{d}\right) \in \mathbb{Z}^{d}, \vec{\sigma}=\left(\sigma_{1}, \ldots, \sigma_{d}\right) \in\{ \pm\}^{d}$ we set $\vec{\sigma} \cdot \vec{\jmath}:=\sigma_{1} j_{1}+\cdots+\sigma_{d} j_{d}$, and, given $\left(u_{j}, \bar{u}_{j}\right)_{j \in \mathbb{Z}}=\left(u_{j}^{+}, u_{j}^{-}\right)_{j \in \mathbb{Z}}$, we define the monomial $u_{\vec{\jmath}}^{\vec{\sigma}}:=u_{j_{1}}^{\sigma_{1}} \cdots u_{j_{d}}^{\sigma_{d}}$ (of degree $d)$.

\subsection{The partial Birkhoff normal form}

We now consider the Hamiltonian (1.4) when $F(s)=s^{4} / 4$ since terms of order five or more will not make any difference, see Remark 7.1.

After a rescaling of the variables (and of the Hamiltonian) it becomes

$$
\begin{aligned}
H & =\sum_{j \in \mathbb{Z}} \lambda_{j} u_{j}^{+} u_{j}^{-}+\sum_{\vec{\jmath} \in \mathbb{Z}^{4}, \vec{\sigma} \in\{ \pm\}^{4}, \vec{\sigma} \cdot \vec{\jmath}=0} u_{\vec{\jmath}}^{\vec{\sigma}}=: N+G \\
& =\sum_{j \in \mathbb{Z}} \lambda_{j} u_{j} \bar{u}_{j}+\sum_{|\alpha|+|\beta|=4, \pi(\alpha, \beta)=0} G_{\alpha, \beta} u^{\alpha} \bar{u}^{\beta}, \quad G_{\alpha, \beta}:=\frac{(|\alpha|+|\beta|) !}{\alpha ! \beta !}=\frac{4 !}{\alpha ! \beta !},
\end{aligned}
$$

where $\left(u^{+}, u^{-}\right)=(u, \bar{u}) \in \ell^{a, p} \times \ell^{a, p}$ for some $a>0, p>1 / 2$, and the momentum is (see (2.86))

$$
\pi(\alpha, \beta)=\sum_{j \in \mathbb{Z}} j\left(\alpha_{j}-\beta_{j}\right) .
$$

Note that $0 \leq G_{\alpha, \beta} \leq 4$ ! (recall $\alpha !=\Pi_{i \in \mathbb{Z}} \alpha_{i}$ !) 
Lemma 7.1. - For all $R>0, N_{0}$ satisfying (3.1), the Hamiltonian $G$ defined in (7.1) belongs to $\mathcal{Q}_{R}^{T}\left(N_{0}, 3 / 2,4\right)$ and

$$
\|G\|_{R, N_{0}, 3 / 2,4}^{T}=\left\|X_{G}\right\|_{R} \lessdot R^{2} .
$$

Proof. - The Hamiltonian vector field $X_{G}:=\left(-\mathrm{i} \partial_{\bar{u}} G, \mathrm{i} \partial_{u} G\right)$ has components

$$
\mathrm{i} \sigma \partial_{u_{l}^{\sigma}} G=\mathrm{i} \sigma \sum_{|\alpha|+|\beta|=3, \pi(\alpha, \beta)=-\sigma l} G_{\alpha, \beta}^{l, \sigma} u^{\alpha} \bar{u}^{\beta}, \quad \sigma= \pm, l \in \mathbb{Z},
$$

where

$$
G_{\alpha, \beta}^{l,+}=\left(\alpha_{l}+1\right) G_{\alpha+e_{l}, \beta}, \quad G_{\alpha, \beta}^{l,-}=\left(\beta_{l}+1\right) G_{\alpha, \beta+e_{l}} .
$$

Note that $0 \leq G_{\alpha, \beta}^{l, \sigma} \leq 5$ ! By Definitions 2.6, 2.8 and (2.2)

$$
\left\|X_{G}\right\|_{R}=\frac{1}{R} \sup _{\|u\|_{a, p},\|\bar{u}\|_{a, p}<R}\left(\sum_{l \in \mathbb{Z}, \sigma= \pm} e^{2 a|l|}\langle l\rangle^{2 p}\left(\sum_{|\alpha|+|\beta|=3, \pi(\alpha, \beta)=-\sigma l} G_{\alpha, \beta}^{l, \sigma}\left|u^{\alpha}\right|\left|\bar{u}^{\beta}\right|\right)^{2}\right)^{1 / 2} .
$$

For each component

$$
\begin{aligned}
& \sum_{|\alpha|+|\beta|=3, \pi(\alpha, \beta)=-\sigma l} G_{\alpha, \beta}^{l, \sigma}\left|u^{\alpha}\right|\left|\bar{u}^{\beta}\right| \lessdot \sum_{\sigma_{1} j_{1}+\sigma_{2} j_{2}+\sigma_{3} j_{3}=-\sigma l}\left|u_{j_{1}}^{\sigma_{1}}\right|\left|u_{j_{2}}^{\sigma_{2}}\right|\left|u_{j_{3}}^{\sigma_{3}}\right| \\
& \lessdot(\tilde{u} * \tilde{u} * \tilde{u})_{-\sigma l}
\end{aligned}
$$

where $\tilde{u}:=\left(\tilde{u}_{l}\right)_{l \in \mathbb{Z}}, \tilde{u}_{j}:=\left|u_{j}\right|+\left|\bar{u}_{j}\right|$, and $*$ denotes the convolution of sequences. Note that $\|\tilde{u}\|_{a, p} \leq\|u\|_{a, p}+\|\bar{u}\|_{a, p}$. Since $\ell^{a, p}$ is a Hilbert algebra, $\|\tilde{u} * \tilde{u} * \tilde{u}\|_{a, p} \lessdot\|\tilde{u}\|_{a, p}^{3}$, and

$$
\text { (7.3) } \begin{aligned}
\left\|X_{G}\right\|_{R} & \lessdot R^{-1} \sup _{\|u\|_{a, p},\|\bar{u}\|_{a, p}<R}\left(\sum_{l \in \mathbb{Z}, \sigma= \pm} e^{2 a|l|}\langle l\rangle^{2 p}\left|(\tilde{u} * \tilde{u} * \tilde{u})_{-\sigma l}\right|^{2}\right)^{1 / 2} \\
& \lessdot R^{-1} \sup _{\|u\|_{a, p},\|\bar{u}\|_{a, p}<R}\|\tilde{u} * \tilde{u} * \tilde{u}\|_{a, p} \lessdot R^{-1} \sup _{\|u\|_{a, p},\|\bar{u}\|_{a, p}<R}\|\tilde{u}\|_{a, p}^{3} \lessdot R^{2} .
\end{aligned}
$$

Moreover $G \in \mathcal{H}_{R}^{\text {null }}$, namely $G$ Poisson commutes with the momentum $\mathcal{M}:=\sum_{j \in \mathbb{Z}} j u_{j} \bar{u}_{j}$, because (see (2.81))

$$
\left\{\mathcal{M}, u_{\vec{\jmath}}^{\vec{\sigma}}\right\}=-\mathrm{i} \vec{\sigma} \cdot \vec{\jmath} u_{\vec{\jmath}}^{\vec{\sigma}} .
$$

We now prove that, for all $N \geq N_{0}$, the projection $\Pi_{N, 3 / 2,4} G \in \mathcal{T}_{R}(N, 3 / 2,4)$. Hence (7.2) follows by (7.3) (see Definition 3.4). By Definition 3.2 (with $g \rightsquigarrow G$, no $(x, y)$-variables and $z=u, \bar{z}=\bar{u}$ ), in particular (3.12), (3.13), we get

$$
\begin{aligned}
\Pi_{N, 3 / 2,4} G & =\sum_{|m|,|n|>3 N / 2, \sigma, \sigma^{\prime}= \pm} G_{m, n}^{\sigma, \sigma^{\prime}}\left(w_{L}\right) u_{m}^{\sigma} \bar{u}_{n}^{\sigma^{\prime}} \text { with } \\
G_{m, n}^{\sigma, \sigma^{\prime}}\left(w_{L}\right) & =\sum_{\substack{j \in \mathbb{Z}|j|\left(\alpha_{j}+\beta_{j}\right)<4 N \\
\pi(\alpha, \beta)=-\sigma m-\sigma^{\prime} n}} G_{\alpha, \beta, m, n}^{\sigma, \sigma^{\prime}} u^{\alpha} \bar{u}^{\beta} \quad \text { and } \\
G_{\alpha, \beta, m, n}^{+,+} & =\frac{1}{2-\delta_{m n}} G_{\alpha+e_{m}+e_{n}, \beta}=\frac{1}{2-\delta_{m n}} \frac{4 !}{\left(1+\delta_{m n}\right) !}=12=G_{\alpha, \beta, m, n}^{-,-} \\
G_{\alpha, \beta, m, n}^{+,-} & =G_{\alpha+e_{m}, \beta+e_{n}}=24=G_{\alpha, \beta, m, n}^{-,+} .
\end{aligned}
$$

These coefficients trivially satisfy (3.15) (with $f \rightsquigarrow G$ ), so $\Pi_{N, 3 / 2,4} G \in \mathcal{T}_{R}(N, 3 / 2,4$ ). 
We now perform a Birkhoff semi-normal form on the tangential sites

$$
\mathcal{I}:=\left\{j_{1}, \ldots, j_{n}\right\} \subset \mathbb{Z}, \quad j_{1}<\cdots<j_{n},
$$

recall (2.84). Let $\mathcal{I}^{c}:=\mathbb{Z} \backslash \mathcal{I}$.

Set

$$
\bar{G}:=\frac{1}{2} \sum_{i \text { or } j \in \mathcal{I}} \bar{G}_{i j} u_{i}^{+} u_{i}^{-} u_{j}^{+} u_{j}^{-}, \quad \bar{G}_{i j}:=12\left(2-\delta_{i j}\right), \quad \hat{G}:=\sum_{\substack{\vec{j} \in \mathbb{Z}^{4}, \vec{\sigma} \in\{+,-\}^{4}, \vec{\sigma} \cdot \vec{\jmath}=0, \vec{\jmath} \in\left(\mathcal{I}^{c}\right)^{4}}} u_{\vec{\jmath}}^{\vec{\sigma}} .
$$

By (7.2) and noting that $\bar{G}, \hat{G}$ are projections of $G$, for $R>0, N_{0}$ satisfying (3.1), we have

$$
\|\bar{G}\|_{R, N_{0}, 3 / 2,4}^{T},\|\hat{G}\|_{R, N_{0}, 3 / 2,4}^{T} \lessdot R^{2} .
$$

Proposition 7.1 (Birkhoff normal form). - For any $\mathcal{I} \subset \mathbb{Z}$ and $\mathrm{m}>0$, there exist $R_{0}>0$ and a real analytic, symplectic change of variables

$$
\Gamma: B_{R / 2} \times B_{R / 2} \subset \ell^{a, p} \times \ell^{a, p} \rightarrow B_{R} \times B_{R} \subset \ell^{a, p} \times \ell^{a, p}, \quad 0<R<R_{0},
$$

that takes the Hamiltonian $H=N+G$ in (7.1) into

$$
H_{\text {Birkhoff }}:=H \circ \Gamma=N+\bar{G}+\hat{G}+K
$$

where $\bar{G}, \hat{G}$ are defined in (7.6) and

$$
K:=\sum_{\substack{\vec{\jmath} \in \mathbb{Z}^{2 d}, \vec{\sigma} \in\{+,-\}^{2 d} \\ d \geq 3, \vec{\sigma} \cdot \vec{\jmath}=0}} K_{\vec{\jmath}, \vec{\sigma}} u_{\vec{\jmath}}^{\vec{\sigma}}
$$

satisfies, for $N_{0}^{\prime}:=N_{0}^{\prime}(\mathrm{m}, \mathcal{I}, L, b)$ large enough,

$$
\|K\|_{R / 2, N_{0}^{\prime}, 2,3}^{T} \lessdot R^{4} \text {. }
$$

The rest of this subsection is devoted to the proof of Proposition 7.1. We start following the strategy of [28]. By (2.81) the Poisson bracket

$$
\left\{N, u_{\vec{\jmath}}^{\vec{\sigma}}\right\}=-\mathrm{i} \vec{\sigma} \cdot \lambda_{\vec{\jmath}} u_{\vec{\jmath}}^{\vec{\sigma}}
$$

where $\lambda_{\vec{\jmath}}:=\left(\lambda_{j_{1}}, \ldots, \lambda_{j_{d}}\right)$ and $\lambda_{j}:=\lambda_{j}(\mathrm{~m}):=\sqrt{j^{2}+\mathrm{m}}$.

The following lemma extends Lemma 4 of [28].

Lemma 7.2 (Small divisors). - Let $\vec{\jmath} \in \mathbb{Z}^{4}, \vec{\sigma} \in\{ \pm\}^{4}$ be such that $\vec{\sigma} \cdot \vec{\jmath}=0$ and (up to permutation of the indices)

$$
\begin{aligned}
& \vec{\jmath}=0, \sum_{i=1}^{4} \sigma_{i} \neq 0, \\
& \text { or } \vec{\jmath}=(0,0, q, q), q \neq 0, \sigma_{1}=\sigma_{2}, \\
& \text { or } \vec{\jmath}=(p, p,-p,-p), p \neq 0, \sigma_{1}=\sigma_{2}, \\
& \text { or } \vec{\jmath} \neq(p, p, q, q) .
\end{aligned}
$$

Then, there exists an absolute constant $c_{*}>0$, such that, for every $\mathrm{m} \in(0, \infty)$,

$$
\left|\vec{\sigma} \cdot \lambda_{\vec{\jmath}}(\mathrm{m})\right| \geq \frac{c_{*} \mathrm{~m}}{\left(n_{0}^{2}+\mathrm{m}\right)^{3 / 2}}>0 \quad \text { where } \quad n_{0}:=\min \left\{\left\langle j_{1}\right\rangle,\left\langle j_{2}\right\rangle,\left\langle j_{3}\right\rangle,\left\langle j_{4}\right\rangle\right\} .
$$

Proof. - In the appendix. 
The map $\Gamma:=\Phi_{F}^{1}$ is obtained as the time- 1 flow generated by the Hamiltonian

$$
F:=-\sum_{\substack{\vec{\jmath} \cdot \vec{\sigma}=0, \vec{\sigma} \cdot \lambda_{\vec{J}} \neq 0 \\ \text { and } \vec{\jmath} \notin\left(\mathcal{I}^{c}\right)^{4}}}(7.12)-(7.15) \frac{\mathrm{i}}{\vec{\sigma} \cdot \lambda_{\vec{\jmath}}} u_{\vec{\jmath}}^{\overrightarrow{\vec{\jmath}}} \text {. }
$$

We notice that the condition $\vec{\jmath} \cdot \vec{\sigma}=0, \vec{\sigma} \cdot \lambda_{\vec{\jmath}} \neq 0$ is equivalent to requiring that $\vec{\jmath} \cdot \vec{\sigma}=0$ and $\vec{\jmath}, \vec{\sigma}$ satisfy (7.12)-(7.15). By Lemma 7.2 there is a constant $\bar{c}>0$ (depending only on $\mathrm{m}$ and $\mathcal{I})$ such that

$$
\vec{\jmath} \cdot \vec{\sigma}=0, \vec{\sigma} \cdot \lambda_{\vec{\jmath}} \neq 0 \text { and } \vec{\jmath} \notin\left(\mathcal{I}^{c}\right)^{4} \quad \Longrightarrow \quad\left|\vec{\sigma} \cdot \lambda_{\vec{\jmath}}\right| \geq \bar{c}>0 .
$$

We have proved that the moduli of the small divisors in (7.17) are uniformly bounded away from zero. Hence $F$ is well defined and, arguing as in Lemma 7.1, we get

$$
\left\|X_{F}\right\|_{R} \lessdot R^{2} \text {. }
$$

Moreover $F \in \mathcal{H}_{R}^{\text {null }}$ because in (7.17) the sum is restricted to $\vec{\sigma} \cdot \vec{\jmath}=0$ (see also (7.4)).

Lemma 7.3. - F in (7.17) solves the homological equation

$$
\{N, F\}+G=\operatorname{ad}_{F}(N)+G=\bar{G}+\hat{G}
$$

where $\bar{G}, \hat{G}$ are defined in (7.6).

Proof. - We claim that the only $\vec{\jmath} \in \mathbb{Z}^{4}, \vec{\sigma} \in\{ \pm\}^{4}$ with $\vec{\jmath} \cdot \vec{\sigma}=0$ which do not satisfy (7.12)-(7.15) have the form

$$
j_{1}=j_{2}, j_{3}=j_{4}, \sigma_{1}=-\sigma_{2}, \sigma_{3}=-\sigma_{4} \text { (or permutations of the indices). }
$$

Indeed:

If $\vec{\jmath}=0, \sum_{i} \sigma_{i}=0$ : the $\sigma_{i}$ are pairwise equal and (7.21) holds.

If $\vec{\jmath}=(0,0, q, q), q \neq 0$, and $\sigma_{1}=-\sigma_{2}$ : by $\vec{\jmath} \cdot \vec{\sigma}=0$ we have also $\sigma_{3}=-\sigma_{4}$ and (7.21) holds.

If $\vec{\jmath}=(p, p,-p,-p), p \neq 0$ and $\sigma_{1}=-\sigma_{2}$ : by $\vec{\jmath} \cdot \vec{\sigma}=0$ we have also $\sigma_{3}=-\sigma_{4}$ and (7.21) holds.

If $j_{1}=j_{2}, j_{3}=j_{4}, j_{1}, j_{3} \neq 0, j_{1} \neq-j_{3}$ :

CASE 1. $j_{1} \neq j_{3}$. Then $0=\vec{\sigma} \cdot \vec{\jmath}=\left(\sigma_{1}+\sigma_{2}\right) j_{1}+\left(\sigma_{3}+\sigma_{4}\right) j_{3}$ implies $\sigma_{1}=-\sigma_{2}, \sigma_{3}=-\sigma_{4}$.

CASE 2. $j_{1}=j_{3}$ and so $j_{1}=j_{2}=j_{3}=j_{4} \neq 0$. Hence $0=\left(\sigma_{1}+\sigma_{2}+\sigma_{3}+\sigma_{4}\right) j_{1}$ and (7.21) follows.

By (7.17) and (7.11) all the monomials in $\{N, F\}$ cancel the monomials of $G$ in (7.1) except for those in $\hat{G}$ (see (7.6)) and those of the form $\left|u_{p}\right|^{2}\left|u_{q}\right|^{2}, p$ or $q \in \mathcal{I}$, which contribute to $\bar{G}$. The expression in (7.6) of $\bar{G}$ follows by counting the multiplicities.

The Hamiltonian $F \in \mathcal{H}_{R}^{\text {null }}$ in (7.17) is quasi-Töplitz:

Lemma 7.4. - Let $R>0$. If $N_{0}:=N_{0}(\mathrm{~m}, \mathcal{I}, L, b)$ is large enough, then $F$ defined in (7.17) belongs to $\mathcal{Q}_{R}^{T}\left(N_{0}, 3 / 2,4\right)$ and

$$
\|F\|_{R, N_{0}, 3 / 2,4}^{T} \lessdot R^{2} .
$$


Proof. - We have to show that $F \in \mathcal{H}_{R}^{\text {null }}$ verifies Definition 3.4. For all $N \geq N_{0}$, we compute, by (7.17) and Definition 3.2 (in particular (3.12)), the projection

$$
\Pi_{N, 3 / 2,4} F=\sum_{\substack{|n|,|m|>C N / 4, \sigma, \sigma^{\prime}= \pm,\left|\sigma m+\sigma^{\prime} n\right|<4 N}} F_{m, n}^{\sigma, \sigma^{\prime}}\left(w^{L}\right) u_{m}^{\sigma} u_{n}^{\sigma^{\prime}}
$$

where

(7.24) $F_{m, n}^{\sigma, \sigma^{\prime}}\left(w^{L}\right):=-12 \mathrm{i}$

$$
\sum_{\substack{|i|+|j|<4 N L, i \text { or } j \in \mathcal{I}, \\ \text { ifj } m=n}} \frac{u_{i}^{\sigma_{i}} u_{j}^{\sigma_{j}}}{\sigma_{i} \lambda_{i}+\sigma_{j} \lambda_{j}+\sigma \lambda_{m}+\sigma^{\prime} \lambda_{n}}
$$

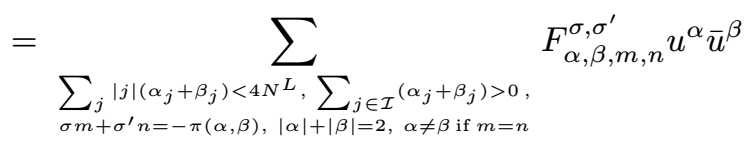

and

$$
F_{\alpha, \beta, m, n}^{\sigma, \sigma^{\prime}}:=-\frac{24 \mathrm{i}}{\alpha ! \beta !} \frac{1}{\lambda_{\alpha, \beta}+\sigma \lambda_{m}+\sigma^{\prime} \lambda_{n}}, \quad \lambda_{\alpha, \beta}:=\sum_{h} \lambda_{h}\left(\alpha_{h}-\beta_{h}\right) .
$$

Notice that in (7.24) the restriction $i \neq j$ if $m=n$ is equivalent to requiring

$$
\left\{(i, j, m, n),\left(\sigma_{i}, \sigma_{j}, \sigma, \sigma^{\prime}\right)\right\} \neq\left\{(i, i, m, m),\left(\sigma_{i},-\sigma_{i}, \sigma,-\sigma\right)\right\},
$$

see Formulas (7.17) and (7.21). Indeed if $m=n,|i|+|j|<4 N^{L}$ and $|m|>C N / 4$ then, by momentum conservation, we have a contribution to (7.24) only if $\sigma=-\sigma^{\prime}$ and hence $|i|=|j|$.

We define the Töplitz approximation

$$
\tilde{F}:=\sum \tilde{F}_{m, n}^{\sigma, \sigma^{\prime}}\left(w^{L}\right) u_{m}^{\sigma} u_{n}^{\sigma^{\prime}} \quad \text { with } \quad \tilde{F}_{m, n}^{\sigma, \sigma^{\prime}}\left(w^{L}\right):=\sum \tilde{F}_{\alpha, \beta, m, n}^{\sigma, \sigma^{\prime}} u^{\alpha} \bar{u}^{\beta}
$$

where the indices in the two sums have the same restrictions as in (7.23), (7.25), respectively, and the coefficients are

$$
\tilde{F}_{\alpha, \beta, m, n}^{\sigma,-\sigma}:=-\frac{24 \mathrm{i}}{\alpha ! \beta !} \frac{1}{\lambda_{\alpha, \beta}+\sigma|m|-\sigma|n|}, \quad \tilde{F}_{\alpha, \beta, m, n}^{\sigma, \sigma}:=0 .
$$

The coefficients in (7.28) are well defined for $N \geq N_{0}$ large enough, because

$$
\begin{gathered}
\left|\lambda_{\alpha, \beta}+\sigma\right| m|-\sigma| n|| \geq\left|\lambda_{\alpha, \beta}+\sigma \lambda_{m}-\sigma \lambda_{n}\right|-\left|\lambda_{m}-\right| m||-\left|\lambda_{n}-\right| n|| \\
\stackrel{(7.18),(7.30)}{\geq} \bar{c}-\frac{\mathrm{m}}{2}\left(\frac{1}{|m|}+\frac{1}{|n|}\right) \geq \bar{c}-\frac{2}{3} \frac{\mathrm{m}}{N_{0}} \geq \frac{\bar{c}}{2}
\end{gathered}
$$

$(\bar{c}$ defined in (7.18)) having used the elementary inequality

$$
\left|\sqrt{n^{2}+\mathrm{m}}-\right| n|| \leq 1 /(2|n|) .
$$

Then (7.27), (7.28), (7.29) imply, arguing as in the proof of Lemma 7.1, that

$$
\left\|X_{\tilde{F}}\right\|_{R} \lessdot R^{2} \text {. }
$$

To prove that $\tilde{F} \in \mathcal{T}_{R}\left(N_{0}, 3 / 2,4\right)$ we have to show (3.15) (with $f \rightsquigarrow \tilde{F}$ ), namely

$$
\tilde{F}_{\alpha, \beta, m, n}^{\sigma, \sigma^{\prime}}=\tilde{F}_{\alpha, \beta}^{\sigma, \sigma^{\prime}}\left(\mathbf{s}(m), \sigma m+\sigma^{\prime} n\right)
$$

with

$$
\tilde{F}_{\alpha, \beta}^{\sigma,-\sigma}(s, h):=-\frac{24 \mathrm{i}}{\alpha ! \beta !} \frac{1}{\lambda_{\alpha, \beta}+s h}, \quad \tilde{F}_{\alpha, \beta}^{\sigma, \sigma}(s, h)=0, \quad s= \pm, h \in \mathbb{Z} .
$$


Recalling (7.28), this is obvious when $\sigma^{\prime}=\sigma$. When $\sigma^{\prime}=-\sigma$ we first note that $\mathbf{s}(m)=\mathbf{s}(n)$. Indeed the restriction on the first sum in (7.27) is (recall (7.23)) $|m|,|n|>3 N / 2$, $|\sigma m-\sigma n|<4 N^{L}$, which implies $\mathbf{s}(m)=\mathbf{s}(n)$ by (3.1). Then

$$
\sigma|m|-\sigma|n|=\sigma \mathbf{s}(m) m-\sigma \mathbf{s}(n) n=\mathbf{s}(m)(\sigma m-\sigma n)
$$

and (7.32) follows. We have proved that $\tilde{F} \in \mathcal{T}_{R}\left(N_{0}, 3 / 2,4\right)$.

The Töplitz defect, defined by (3.29), is

$$
\hat{F}:=\sum \hat{F}_{m, n}^{\sigma, \sigma^{\prime}}\left(w^{L}\right) u_{m}^{\sigma} u_{n}^{\sigma^{\prime}} \quad \text { with } \quad \hat{F}_{m, n}^{\sigma, \sigma^{\prime}}\left(w^{L}\right):=\sum \hat{F}_{\alpha, \beta, m, n}^{\sigma, \sigma^{\prime}} u^{\alpha} \bar{u}^{\beta}
$$

where the indices in the two sums have the same restrictions as in (7.23)-(7.25), and

$$
\begin{aligned}
\hat{F}_{\alpha, \beta, m, n}^{\sigma, \sigma} & =-\frac{24 \mathrm{i}}{\alpha ! \beta !} \frac{N}{\lambda_{\alpha, \beta}+\sigma \lambda_{m}+\sigma \lambda_{n}} \\
\hat{F}_{\alpha, \beta, m, n}^{\sigma,-\sigma} & =-N \frac{24 \mathrm{i}}{\alpha ! \beta !}\left(\frac{1}{\lambda_{\alpha, \beta}+\sigma \lambda_{m}-\sigma \lambda_{n}}-\frac{1}{\lambda_{\alpha, \beta}+\sigma|m|-\sigma|n|}\right) \\
& =\frac{24 \mathrm{i}}{\alpha ! \beta !} \frac{N \sigma\left(\lambda_{m}-|m|-\lambda_{n}+|n|\right)}{\left(\lambda_{\alpha, \beta}+\sigma \lambda_{m}-\sigma \lambda_{n}\right)\left(\lambda_{\alpha, \beta}+\sigma|m|-\sigma|n|\right)} .
\end{aligned}
$$

We now prove that the coefficients in (7.34)-(7.35) are bounded by a constant independent of $N$.

The coefficients in (7.34) are bounded because

$$
\left|\lambda_{\alpha, \beta}\right| \leq \sum_{h} \lambda_{h}\left(\left|\alpha_{h}\right|+\left|\beta_{h}\right|\right) \leq \sum_{h}|h|\left(\left|\alpha_{h}\right|+\left|\beta_{h}\right|\right)+\sqrt{\mathrm{m}} \sum_{h}\left(\left|\alpha_{h}\right|+\left|\beta_{h}\right|\right) \leq 4 N^{L}+2 \sqrt{\mathrm{m}}
$$

by (7.26)-(7.25) (note that $\lambda_{h} \leq|h|+\sqrt{\mathrm{m})}$ and

$$
\left|\lambda_{\alpha, \beta}+\sigma \lambda_{m}+\sigma \lambda_{n}\right| \geq\left|\lambda_{m}+\lambda_{n}\right|-\left|\lambda_{\alpha, \beta}\right| \geq 3 N-4 N^{L}-2 \sqrt{\mathrm{m}} \geq 3 N / 2
$$

for $N \geq N_{0}$ large enough.

The coefficients in (7.35) are bounded by (7.18), (7.29), and

$$
N\left|\lambda_{m}-\right| m\left|-\lambda_{n}+\right| n|| \stackrel{(7.30)}{\leq} N \frac{\mathrm{m}}{2}\left(\frac{1}{|m|}+\frac{1}{|m|}\right) \leq \frac{2}{3} \mathrm{~m} .
$$

Hence arguing as in the proof of Lemma 7.1 we get

$$
\left\|X_{\hat{F}}\right\|_{R} \lessdot R^{2} \text {. }
$$

In conclusion, (7.19), (7.31), (7.36) imply (7.22) (recall (3.30)).

Proof of Proposition 7.1 completed. - We have

$$
\begin{aligned}
e^{\operatorname{ad}_{F}} H & =e^{\operatorname{ad}_{F}} N+e^{\operatorname{ad}_{F}} G=N+\{N, F\}+\sum_{i \geq 2} \frac{1}{i !} \operatorname{ad}_{F}^{i}(N)+G+\sum_{i \geq 1} \frac{1}{i !} \operatorname{ad}_{F}^{i}(G) \\
& \stackrel{(7.20)}{=} N+\bar{G}+\hat{G}+\sum_{i \geq 1} \frac{1}{(i+1) !} \operatorname{ad}_{F}^{i}\left(\operatorname{ad}_{F}(N)\right)+\sum_{i \geq 1} \frac{1}{i !} \operatorname{ad}_{F}^{i}(G) \\
& =N+\bar{G}+\hat{G}+K
\end{aligned}
$$

where, using again (7.20),

$$
K:=\sum_{i \geq 1} \frac{1}{(i+1) !} \operatorname{ad}_{F}^{i}(\bar{G}+\hat{G}-G)+\sum_{i \geq 1} \frac{1}{i !} \operatorname{ad}_{F}^{i} G=: K_{1}+K_{2} .
$$


Proof of (7.9). - We claim that in the expansion of $K$ in (7.37) there are only monomials $u_{\vec{\jmath}}^{\vec{\sigma}}$ with $\vec{\jmath} \in \mathbb{Z}^{2 d}, \vec{\sigma} \in\{+,-\}^{2 d}, d \geq 3$. Indeed $F, G, \bar{G}, \hat{G}$ contain only monomials of degree four and, for any monomial $\mathfrak{m}, \operatorname{ad}_{F}(\mathfrak{m})$ contains only monomials of degree equal to the $\operatorname{deg}(\mathfrak{m})+2$. The restriction $\vec{\sigma} \cdot \vec{\jmath}=0$ follows by the Jacobi identity (2.82), since $F, G, \bar{G}, \hat{G}$ preserve momentum, i.e., Poisson commutes with $M$.

Proof of (7.10). - We apply Proposition 3.2 with (no $(x, y)$ variables and)

$$
\begin{gathered}
f \rightsquigarrow F, \quad g \rightsquigarrow\left\{\begin{array}{ll}
\bar{G}+\hat{G}-G & \text { for } K_{1}, \\
G \quad & \text { for } K_{2},
\end{array} \quad r \rightsquigarrow R, \quad r^{\prime} \rightsquigarrow R / 2, \quad \delta \rightsquigarrow 1 / 2,\right. \\
\theta \rightsquigarrow 3 / 2, \quad \theta^{\prime} \rightsquigarrow 2, \quad \mu \rightsquigarrow 4, \quad \mu^{\prime} \rightsquigarrow 3,
\end{gathered}
$$

$N_{0}$ defined in Lemma 7.4 and $N_{0}^{\prime} \geq N_{0}$ satisfying (3.64) and

$$
\kappa\left(N_{0}^{\prime}\right)^{b-L} \ln N_{0}^{\prime} \leq 1, \quad(6+\kappa)\left(N_{0}^{\prime}\right)^{L-1} \ln N_{0}^{\prime} \leq 1 / 2 .
$$

Note that (3.65) follows by (7.38). By (7.22), the assumption (3.63) is verified for every $0<R<R_{0}$, with $R_{0}$ small enough. Then Proposition 3.2 applies and (7.10) follows by (3.67) (with $h \rightsquigarrow 1$ ), (7.2), (7.22) and (7.7).

\subsection{Action-angle variables}

We introduce action-angle variables on the tangential sites $\mathcal{I}:=\left\{j_{1}, \ldots, j_{n}\right\}$ (see (7.5)) via the analytic and symplectic map

$$
\Phi(x, y, z, \bar{z} ; \xi):=(u, \bar{u})
$$

defined by

$$
u_{\mathrm{j}_{l}}:=\sqrt{\xi_{l}+y_{l}} e^{\mathrm{i} x_{l}}, \bar{u}_{\mathrm{j}_{l}}:=\sqrt{\xi_{l}+y_{l}} e^{-\mathrm{i} x_{l}}, l=1, \ldots, n, u_{j}:=z_{j}, \bar{u}_{j}:=\bar{z}_{j}, j \in \mathbb{Z} \backslash \mathcal{I} .
$$

Let

$$
\mathcal{O}_{\rho}:=\left\{\xi \in \mathbb{R}^{n}: \frac{\rho}{2} \leq \xi_{l} \leq \rho, l=1, \ldots, n\right\} .
$$

Lemma 7.5 (Domains). - Let $r, R, \rho>0$ satisfy

$$
16 r^{2}<\rho, \quad \rho=C_{*} R^{2} \quad \text { with } \quad C_{*}^{-1}:=48 n \kappa^{2 p} e^{2(s+a \kappa)} .
$$

Then, for all $\xi \in \mathcal{O}_{\rho} \cup \mathcal{O}_{2 \rho}$, the map

$$
\Phi(\cdot ; \xi): D(s, 2 r) \rightarrow \mathcal{D}(R / 2):=B_{R / 2} \times B_{R / 2} \subset \ell^{a, p} \times \ell^{a, p}
$$

is well defined and analytic $(D(s, 2 r)$ is defined in (2.5) and $\kappa$ in (3.1)).

Proof. - Note first that for $(x, y, z, \bar{z}) \in D(s, 2 r)$ we have (see (2.6)) that $\left|y_{l}\right|<4 r^{2} \stackrel{\text { (7.42) }}{<}$ $\rho / 4<\xi_{l}, \forall \xi \in \mathcal{O}_{\rho} \cup \mathcal{O}_{2 \rho}$. Then the map $y_{l} \mapsto \sqrt{\xi_{l}+y_{l}}$ is well defined and analytic. Moreover, for $\xi_{l} \leq 2 \rho,\left|j_{l}\right| \leq \kappa, x \in \mathbb{T}_{s}^{n},\|z\|_{a, p}<2 r$, we get

$$
\begin{aligned}
\|u(x, y, z, \bar{z} ; \xi)\|_{a, p}^{2} & \stackrel{(7.39)}{=} \sum_{l=1}^{n}\left(\xi_{l}+y_{l}\right)\left|e^{2 \mathrm{i} x_{l}}\right|\left|j_{l}\right|^{2 p} e^{2 a\left|j_{l}\right|}+\sum_{j \in \mathbb{Z} \backslash \mathcal{I}}\left|z_{j}\right|^{2}\langle j\rangle^{2 p} e^{2 a|j|} \\
& \leq n\left(2 \rho+\frac{\rho}{4}\right) e^{2 s} \kappa^{2 p} e^{2 a \kappa}+4 r^{2} \stackrel{(7.42)}{<} R^{2} / 4
\end{aligned}
$$

$4^{\mathrm{e}}$ SÉRIE - TOME $46-2013$ - No 2 
proving (7.43) (the bound for $\bar{u}$ is the same).

Given a function $F: \mathcal{D}(R / 2) \rightarrow \mathbb{C}$, the previous lemma shows that the composite map $F \circ \Phi: D(s, 2 r) \rightarrow \mathbb{C}$. The main result of this section is Proposition 7.2: if $F$ is quasi-Töplitz in the variables $(u, \bar{u})$ then the composite $F \circ \Phi$ is quasi-Töplitz in the variables $(x, y, z, \bar{z})$ (see Definition 3.4).

We write

$$
F=\sum_{\alpha, \beta} F_{\alpha, \beta} \mathfrak{m}_{\alpha, \beta}, \quad \mathfrak{m}_{\alpha, \beta}:=\left(u^{(1)}\right)^{\alpha^{(1)}}\left(\bar{u}^{(1)}\right)^{\beta^{(1)}}\left(u^{(2)}\right)^{\alpha^{(2)}}\left(\bar{u}^{(2)}\right)^{\beta^{(2)}}
$$

where

$$
u=\left(u^{(1)}, u^{(2)}\right), \quad u^{(1)}:=\left\{u_{j}\right\}_{j \in \mathcal{I}}, u^{(2)}:=\left\{u_{j}\right\}_{j \in \mathbb{Z} \backslash \mathcal{I}}, \quad \text { similarly for } \bar{u},
$$

and

$(\alpha, \beta)=\left(\alpha^{(1)}+\alpha^{(2)}, \beta^{(1)}+\beta^{(2)}\right), \quad\left(\alpha^{(1)}, \beta^{(1)}\right):=\left\{\alpha_{j}, \beta_{j}\right\}_{j \in \mathcal{I}}, \quad\left(\alpha^{(2)}, \beta^{(2)}\right):=\left\{\alpha_{j}, \beta_{j}\right\}_{j \in \mathbb{Z} \backslash \mathcal{I}}$.

We define

$$
\mathcal{H}_{R}^{d}:=\left\{F \in \mathcal{H}_{R}: F=\sum_{\left|\alpha^{(2)}+\beta^{(2)}\right| \geq d} F_{\alpha, \beta} u^{\alpha} \bar{u}^{\beta}\right\}
$$

Proposition 7.2 (Quasi-Töplitz). - Let $N_{0}, \theta, \mu, \mu^{\prime}$ satisfying (3.1) and

$$
\left(\mu^{\prime}-\mu\right) N_{0}^{L}>N_{0}^{b}, \quad N_{0} 2^{-\frac{N_{0}^{b}}{2 \kappa}+1}<1 .
$$

If $F \in \mathcal{Q}_{R / 2}^{T}\left(N_{0}, \theta, \mu^{\prime}\right) \cap \mathcal{H}_{R / 2}^{d}$ with $d=0,1$, then $f:=F \circ \Phi \in \mathcal{Q}_{s, r}^{T}\left(N_{0}, \theta, \mu\right)$ and

$$
\|f\|_{s, r, N_{0}, \theta, \mu, \mathcal{O}_{\rho}}^{T} \lessdot(8 r / R)^{d-2}\|F\|_{R / 2, N_{0}, \theta, \mu^{\prime}}^{T} .
$$

The rest of this section is devoted to the proof of Proposition 7.2. Introducing the actionangle variables (7.40) in (7.44), and using the Taylor expansion

$$
(1+t)^{\gamma}=\sum_{h \geq 0}\left(\begin{array}{l}
\gamma \\
h
\end{array}\right) t^{h}, \quad\left(\begin{array}{l}
\gamma \\
0
\end{array}\right):=1,\left(\begin{array}{l}
\gamma \\
h
\end{array}\right):=\frac{\gamma(\gamma-1) \ldots(\gamma-h+1)}{h !}, h \geq 1
$$

we get

$$
f:=F \circ \Phi=\sum_{k, i, \alpha^{(2)}, \beta^{(2)}} f_{k, i, \alpha^{(2)}, \beta^{(2)}} e^{\mathrm{i} k \cdot x} y^{i} z^{\alpha^{(2)}} \bar{z}^{\beta^{(2)}}
$$

with Taylor-Fourier coefficients

$$
f_{k, i, \alpha^{(2)}, \beta^{(2)}}:=\sum_{\alpha^{(1)}-\beta^{(1)}=k} F_{\alpha, \beta} \prod_{l=1}^{n} \xi_{l}^{\frac{\alpha_{l}^{(1)}+\beta_{l}^{(1)}}{2}}-i_{l}\left(\begin{array}{c}
\frac{\alpha_{l}^{(1)}+\beta_{l}^{(1)}}{2} \\
i_{l}
\end{array}\right) .
$$

We need an upper bound on the binomial coefficients.

Lemma 7.6. - For $|t|<1 / 2$ we have

$$
\text { (i) } \sum_{h \geq 0}|t|^{h}\left|\left(\begin{array}{c}
\frac{k}{2} \\
h
\end{array}\right)\right| \leq 2^{k}, \forall k \geq 0, \quad \text { (ii) } \sum_{h \geq 1}|t|^{h}\left|\left(\begin{array}{c}
\frac{k}{2} \\
h
\end{array}\right)\right| \leq 3^{k}|t|, \forall k \geq 1 .
$$


Proof. - By (7.49) and the definition of majorant (see (2.11)) we have

$$
\sum_{h \geq 0}\left|\left(\begin{array}{c}
\frac{k}{2} \\
h
\end{array}\right)\right| t^{h}=M(1+t)^{\frac{k}{2}} \stackrel{(2.39)}{\prec}\left(M(1+t)^{\frac{1}{2}}\right)^{k}=\left(\sum_{h \geq 0}\left|\left(\begin{array}{c}
\frac{1}{2} \\
h
\end{array}\right)\right| t^{h}\right)^{k} \prec\left(\sum_{h \geq 0} t^{h}\right)^{k}
$$

because $\left|\left(\begin{array}{l}\frac{1}{2} \\ h\end{array}\right)\right| \leq 1$ by (7.49). For $|t|<1 / 2$ the bound (7.53) implies (7.52)-(i). Then

$$
\begin{aligned}
\sum_{h \geq 1}|t|^{h}\left|\left(\begin{array}{c}
\frac{k}{2} \\
h
\end{array}\right)\right| \leq|t| \sum_{h \geq 0}|t|^{h}\left|\left(\begin{array}{c}
\frac{k}{2} \\
h+1
\end{array}\right)\right| & \stackrel{(7.49)}{=}|t| \sum_{h \geq 0}|t|^{h}\left|\left(\begin{array}{c}
\frac{k}{2} \\
h
\end{array}\right)\right| \frac{\left|\frac{k}{2}-h\right|}{h+1} \leq k|t| \sum_{h \geq 0}|t|^{h}\left|\left(\begin{array}{c}
\frac{k}{2} \\
h
\end{array}\right)\right| \stackrel{\text { (7.52)-(i) }}{\leq} \stackrel{k}{k} 2^{k}|t|
\end{aligned}
$$

which implies (7.52)-(ii) for $k \geq 1$.

Lemma 7.7 ( $M$-regularity). - If $F \in \mathcal{H}_{R / 2}^{d}$ then $f:=F \circ \Phi \in \mathcal{H}_{s, 2 r}$ and

$$
\left\|X_{f}\right\|_{s, 2 r, \mathcal{O}_{\rho} \cup \mathcal{O}_{2 \rho}} \lessdot(8 r / R)^{d-2}\left\|X_{F}\right\|_{R / 2} .
$$

Moreover if $F$ preserves momentum then so does $F \circ \Phi$.

Proof. - We first bound the majorant norm (recall also (7.46))

$\|f\|_{s, 2 r, \mathcal{O}_{\rho} \cup \mathcal{O}_{2 \rho}} \stackrel{(7.50),(7.46)}{:=} \sup _{\xi \in \mathcal{O}_{\rho} \cup \mathcal{O}_{2 \rho}(y, z, \bar{z}) \in D(2 r)} \sup _{k, i,\left|\alpha^{(2)}+\beta^{(2)}\right| \geq d}\left|f_{k, i, \alpha^{(2)}, \beta^{(2)}}\right| e^{|k| s}\left|y^{i}\right|\left|z^{\alpha^{(2)}} \| \bar{z}^{\beta^{(2)}}\right|$.

Fix $\alpha^{(2)}, \beta^{(2)}$. Since for all $\xi \in \mathcal{O}_{\rho} \cup \mathcal{O}_{2 \rho}, y \in B_{(2 r)^{2}}$, we have $\left|y_{l} / \xi_{l}\right|<1 / 2$ by (7.42), we have

$$
\begin{aligned}
\sum_{k} e^{|k| s} \sum_{i}\left|f_{k, i, \alpha^{(2)}, \beta^{(2)}}\right||y|^{i} & \stackrel{(7.51)}{\leq} \sum_{\alpha^{(1)}, \beta^{(1)}} e^{s\left(\left|\alpha^{(1)}\right|+\left|\beta^{(1)}\right|\right)}\left|F_{\alpha, \beta}\right| \xi^{\frac{\alpha^{(1)}+\beta^{(1)}}{2}} \prod_{l=1}^{n} \sum_{i_{l} \geq 0}\left|\frac{y_{l}}{\xi_{l}}\right|^{i_{l}}\left|\left(\begin{array}{c}
\frac{\alpha_{l}^{(1)}+\beta_{l}^{(1)}}{2} \\
i_{l}
\end{array}\right)\right| \\
& \stackrel{(7.52)}{\leq} \sum_{\alpha^{(1)}, \beta^{(1)}} e^{s\left(\left|\alpha^{(1)}\right|+\left|\beta^{(1)}\right|\right)}\left|F_{\alpha, \beta}\right| \xi^{\frac{\alpha^{(1)}+\beta^{(1)}}{2}} \prod_{l=1}^{n} 2^{\alpha_{l}^{(1)}+\beta_{l}^{(1)}} \\
& \leq \sum_{\alpha^{(1)}, \beta^{(1)}} e^{s\left(\left|\alpha^{(1)}\right|+\left|\beta^{(1)}\right|\right)}\left|F_{\alpha, \beta}\right|(2 \rho)^{\frac{\left|\alpha^{(1)}\right|+\left|\beta^{(1)}\right|}{2}} 2^{\left|\alpha^{(1)}\right|+\left|\beta^{(1)}\right|} \\
& =\sum_{\alpha^{(1), \beta^{(1)}}}\left(2 e^{s} \sqrt{2 \rho}\right)^{\left|\alpha^{(1)}\right|+\left|\beta^{(1)}\right|}\left|F_{\alpha, \beta}\right|
\end{aligned}
$$

Then, substituting in (7.55),

$$
\begin{aligned}
\|f\|_{s, 2 r, \mathcal{O}_{\rho} \cup \mathcal{O}_{2 \rho}} & \leq \sup _{\|z\|_{a, p},\|\bar{z}\|_{a, p}<2 r} G(z, \bar{z}) \quad \text { where } \\
G(z, \bar{z}) & :=\sum_{\left|\alpha^{(2)}+\beta^{(2)}\right| \geq d}\left(2 e^{s} \sqrt{2 \rho}\right)^{\left|\alpha^{(1)}\right|+\left|\beta^{(1)}\right|}\left|F_{\alpha, \beta}\left\|z^{\alpha^{(2)}}\right\| \bar{z}^{\beta^{(2)}}\right| .
\end{aligned}
$$

By (7.42), for all $\|z\|_{a, p},\|\bar{z}\|_{a, p}<2 r$, the vector $\left(u^{*}, \bar{u}^{*}\right)$ defined by

(7.62) $u_{j}^{*}=\bar{u}_{j}^{*}:=2 e^{s} \sqrt{2 \rho}, j \in \mathcal{I}, \quad u_{j}^{*}:=(R /(8 r))\left|z_{j}\right|, \bar{u}_{j}^{*}:=(R /(8 r))\left|\bar{z}_{j}\right|, j \in \mathbb{Z} \backslash \mathcal{I}$ 
belongs to $B_{R / 2} \times B_{R / 2}$. Then, by (7.61), recalling (2.11), Definition 2.2 (and since $R /(8 r)>1$ by $(7.42))$,

$$
G(z, \bar{z}) \leq(8 r / R)^{d}(M F)\left(u^{*}, \bar{u}^{*}\right) \leq(8 r / R)^{d}\|F\|_{R / 2}, \quad \forall\|z\|_{a, p},\|\bar{z}\|_{a, p}<2 r .
$$

Hence by $(7.60)$

$$
\|f\|_{s, 2 r, \mathcal{O}_{\rho} \cup \mathcal{O}_{2 \rho}} \leq(8 r / R)^{d}\|F\|_{R / 2} .
$$

This shows that $f$ is $M$-regular. Similarly we get

$$
\left\|\partial_{z} f\right\|_{s, 2 r, \mathcal{O}_{\rho} \cup \mathcal{O}_{2 \rho}} \leq\left\|\partial_{u^{(2)}} F\right\|_{R / 2}(8 r / R)^{d-1}, \text { same for } \partial_{\bar{z}} .
$$

Moreover, by the chain rule, and (7.63)

$$
\begin{aligned}
\left\|\partial_{x_{i}} f\right\|_{s, 2 r, \mathcal{O}_{\rho} \cup \mathcal{O}_{2 \rho}} & \leq\left(\left\|\partial_{u_{i}^{(1)}} F\right\|_{R / 2}+\left\|\partial_{\bar{u}_{i}^{(1)}} F\right\|_{R / 2}\right) \sqrt{2 \rho+\rho / 4} e^{s}(8 r / R)^{d} \\
\left\|\partial_{y_{i}} f\right\|_{s, 2 r, \mathcal{O}_{\rho} \cup \mathcal{O}_{2 \rho}} & \leq\left(\left\|\partial_{u_{i}^{(1)}} F\right\|_{R / 2}+\left\|\partial_{\bar{u}_{i}^{(1)}} F\right\|_{R / 2}\right) \frac{e^{s}}{\sqrt{\rho / 2-\rho / 4}}(8 r / R)^{d} .
\end{aligned}
$$

Then (7.54) follows by (7.42) (recalling (2.2)).

Definition 7.1. - For a monomial $\mathfrak{m}_{\alpha, \beta}:=\left(u^{(1)}\right)^{\alpha^{(1)}}\left(\bar{u}^{(1)}\right)^{\beta^{(1)}}\left(u^{(2)}\right)^{\alpha^{(2)}}\left(\bar{u}^{(2)}\right)^{\beta^{(2)}}$ (as in (7.44)) we set

$$
\mathfrak{p}\left(\mathfrak{m}_{\alpha, \beta}\right):=\sum_{l=1}^{n}\left\langle\mathfrak{j}_{l}\right\rangle\left(\alpha_{\mathfrak{j}_{l}}^{(1)}+\beta_{\mathbf{j}_{l}}^{(1)}\right), \quad\langle j\rangle:=\max \{1,|j|\} .
$$

For any $F$ as in (7.44), $K \in \mathbb{N}$, we define the projection

$$
\Pi_{\mathfrak{p} \geq K} F:=\sum_{\mathfrak{p}\left(\mathfrak{m}_{\alpha, \beta}\right) \geq K} F_{\alpha, \beta} \mathfrak{m}_{\alpha, \beta}, \quad \Pi_{\mathfrak{p}<K}:=I-\Pi_{\mathfrak{p} \geq K} .
$$

Lemma 7.8. - Let $F \in \mathcal{H}_{R / 2}$. Then

$$
\left\|X_{\left(\Pi_{\mathfrak{p} \geq K} F\right) \circ \Phi}\right\|_{s, r, \mathcal{O}_{\rho}} \leq 2^{-\frac{K}{2 \kappa}+1}\left\|X_{F \circ \Phi}\right\|_{s, 2 r, \mathcal{O}_{2 \rho}} .
$$

Proof. - For each monomial $\mathfrak{m}_{\alpha, \beta}$ as in (7.44) with $\mathfrak{p}\left(\mathfrak{m}_{\alpha, \beta}\right) \geq K$ we have

$$
\left|\alpha^{(1)}+\beta^{(1)}\right| \stackrel{(7.45)}{=} \sum_{l=1}^{n} \alpha_{\mathfrak{j}_{l}}^{(1)}+\beta_{\mathbf{j}_{l}}^{(1)} \stackrel{(3.1)}{\geq} \kappa^{-1} \sum_{l=1}^{n}\left\langle\mathfrak{j}_{l}\right\rangle\left(\alpha_{\mathfrak{j}_{l}}^{(1)}+\beta_{\mathfrak{j}_{l}}^{(1)}\right) \stackrel{(7.65)}{=} \kappa^{-1} \mathfrak{p}\left(\mathfrak{m}_{\alpha, \beta}\right) \geq \kappa^{-1} K
$$

and then, $\forall \xi \in \mathcal{O}_{\rho}, y \in B_{r^{2}}$,

$$
\begin{aligned}
\left|\left(\mathfrak{m}_{\alpha, \beta} \circ \Phi\right)(x, y, z, \bar{z} ; \xi)\right| & \stackrel{(7.40)}{=}\left|(\xi+y)^{\frac{\alpha^{(1)}+\beta^{(1)}}{2}} e^{\mathrm{i}\left(\alpha^{(1)}-\beta^{(1)}\right) \cdot x} z^{\alpha^{(2)}} \bar{z}^{\beta^{(2)}}\right| \\
& =2^{-\frac{\mid \alpha(1)+\beta^{(1)}}{2}}\left|(2 \xi+2 y)^{\frac{\alpha^{(1)}+\beta^{(1)}}{2}} e^{\mathrm{i}\left(\alpha^{(1)}-\beta^{(1)}\right) \cdot x} z^{\alpha^{(2)}} \bar{z}^{\beta^{(2)}}\right| \\
& \leq 2^{-\frac{K}{2 \kappa}}\left|\left(\mathfrak{m}_{\alpha, \beta} \circ \Phi\right)(x, 2 y, z, \bar{z} ; 2 \xi)\right| .
\end{aligned}
$$

The bound (7.67) for the Hamiltonian vector field follows applying the above rescaling argument to each component, and noting that the derivatives with respect to $y$ in the vector field decrease the degree in $\xi$ by one. 
Let $N_{0}, \theta, \mu, \mu^{\prime}$ be as in Proposition 7.2. For $N \geq N_{0}$ and $F \in \mathcal{H}_{R / 2}$ we set

$$
f^{*}:=\Pi_{N, \theta, \mu}\left(\left(F-\Pi_{N, \theta, \mu^{\prime}} F\right) \circ \Phi\right) .
$$

Note that $\Pi_{N, \theta, \mu^{\prime}}$ is the projection on the bilinear functions in the variables $u, \bar{u}$, and $\Pi_{N, \theta, \mu}$ in the variables $x, y, z, \bar{z}$.

Lemma 7.9. - We have

$$
\left\|X_{f *}\right\|_{s, r, \mathcal{O}_{\rho}} \leq 2^{-\frac{N^{b}}{2 \kappa}+1}\left\|X_{F \circ \Phi}\right\|_{s, 2 r, \mathcal{O}_{2 \rho}} .
$$

Proof. - We first claim that if $F=\mathfrak{m}_{\alpha, \beta}$ is a monomial as in (7.44) with $\mathfrak{p}\left(\mathfrak{m}_{\alpha, \beta}\right)<N^{b}$ then $f^{*}=0$.

CASE 1. $\mathfrak{m}_{\alpha, \beta}$ is $\left(N, \theta, \mu^{\prime}\right)$-bilinear, see Definition 3.2. Then $\Pi_{N, \theta, \mu^{\prime}} \mathfrak{m}_{\alpha, \beta}=\mathfrak{m}_{\alpha, \beta}$ and $f^{*}=0$, see (7.69).

CASE 2. $\mathfrak{m}_{\alpha, \beta}$ is not $\left(N, \theta, \mu^{\prime}\right)$-bilinear. Then $\Pi_{N, \theta, \mu^{\prime}} \mathfrak{m}_{\alpha, \beta}=0$ and $f^{*}=\Pi_{N, \theta, \mu}\left(\mathfrak{m}_{\alpha, \beta} \circ \Phi\right)$, see (7.69). We claim that $\mathfrak{m}_{\alpha, \beta} \circ \Phi$ is not $(N, \theta, \mu)$-bilinear, and so $f^{*}=\Pi_{N, \theta, \mu}\left(\mathfrak{m}_{\alpha, \beta} \circ \Phi\right)=0$. Indeed,

$$
\mathfrak{m}_{\alpha, \beta} \circ \Phi=(\xi+y)^{\frac{\alpha^{(1)}+\beta^{(1)}}{2}} e^{\mathrm{i}\left(\alpha^{(1)}-\beta^{(1)}\right) \cdot x} z^{\alpha^{(2)}} \bar{z}^{\beta^{(2)}}
$$

is $(N, \theta, \mu)$-bilinear if and only if (see Definitions 3.2 and 3.1)

$$
\begin{gathered}
z^{\alpha^{(2)}} \bar{z}^{\beta^{(2)}}=z^{\tilde{\alpha}^{(2)}} \bar{z}^{\tilde{\beta}^{(2)}} z_{m}^{\sigma} z_{n}^{\sigma^{\prime}}, \\
\sum_{j \in \mathbb{Z} \backslash \mathcal{I}}|j|\left(\tilde{\alpha}_{j}^{(2)}+\tilde{\beta}_{j}^{(2)}\right)<\mu N^{L}, \quad|m|,|n|>\theta N, \quad\left|\alpha^{(1)}-\beta^{(1)}\right|<N^{b} .
\end{gathered}
$$

We deduce the contradiction that

$$
\mathfrak{m}_{\alpha, \beta}=\left(u^{(1)}\right)^{\alpha^{(1)}}\left(\bar{u}^{(1)}\right)^{\beta^{(1)}}\left(u^{(2)}\right)^{\tilde{\alpha}^{(2)}}\left(\bar{u}^{(2)}\right)^{\tilde{\beta}^{(2)}} u_{m}^{\sigma} u_{n}^{\sigma^{\prime}}
$$

is $\left(N, \theta, \mu^{\prime}\right)$-bilinear because (recall that we suppose $\mathfrak{p}\left(\mathfrak{m}_{\alpha, \beta}\right)<N^{b}$ )

$$
\sum_{l=1}^{n}\left|j_{l}\right|\left(\alpha_{\mathfrak{j}_{l}}^{(1)}+\beta_{\mathfrak{j}_{l}}^{(1)}\right)+\sum_{j \in \mathbb{Z} \backslash \mathcal{I}}|j|\left(\tilde{\alpha}_{j}^{(2)}+\tilde{\beta}_{j}^{(2)}\right) \stackrel{(7.65),(7.72)}{<} \mathfrak{p}\left(\mathfrak{m}_{\alpha, \beta}\right)+\mu N^{L}<N^{b}+\mu N^{L} \stackrel{(7.47)}{<} \mu^{\prime} N^{L} .
$$

$\left(N, \theta, \mu^{\prime}\right)$-bilinear, a contradiction. For the general case, we divide $F=\Pi_{\mathfrak{p}<N^{b}} F+\Pi_{\mathfrak{p} \geq N^{b}} F$. By the above claim

$$
f^{*}=\Pi_{N, \theta, \mu}\left(\left(\left(I d-\Pi_{N, \theta, \mu^{\prime}}\right) \Pi_{\mathfrak{p} \geq N^{b}} F\right) \circ \Phi\right)=\Pi_{N, \theta, \mu}\left(\left(\Pi_{\mathfrak{p} \geq N^{b}}\left(I d-\Pi_{N, \theta, \mu^{\prime}}\right) F\right) \circ \Phi\right) .
$$

Finally, (7.70) follows by (2.80) and applying Lemma 7.8 to $\left(\Pi_{\mathfrak{p} \geq N^{b}}\left(I d-\Pi_{N, \theta, \mu^{\prime}}\right) F\right) \circ \Phi$.

Lemma 7.10. - Let $F \in \mathcal{T}_{R / 2}\left(N, \theta, \mu^{\prime}\right)$ with $\Pi_{\mathfrak{p} \geq N^{b}} F=0$. Then $F \circ \Phi(\cdot ; \xi) \in$ $\mathcal{T}_{s, 2 r}\left(N, \theta, \mu^{\prime}\right), \forall \xi \in \mathcal{O}_{\rho} \cup \mathcal{O}_{2 \rho}$. 
Proof. - Recalling Definition 3.3 we have

$$
F=\sum_{|m|,|n|>\theta N, \sigma, \sigma^{\prime}= \pm} F^{\sigma, \sigma^{\prime}}\left(\mathbf{s}(m), \sigma m+\sigma^{\prime} n\right) u_{m}^{\sigma} u_{n}^{\sigma^{\prime}} \text { with } F^{\sigma, \sigma^{\prime}}(\varsigma, h) \in \mathcal{L}_{R / 2}\left(N, \mu^{\prime}, h\right) .
$$

Composing with the map $\Phi$ in (7.40), since $m, n \notin \mathcal{I}$, we get

$$
F \circ \Phi=\sum_{\sigma, \sigma^{\prime}= \pm,|m|,|n|>\theta N} F^{\sigma, \sigma^{\prime}}\left(\mathrm{s}(m), \sigma m+\sigma^{\prime} n\right) \circ \Phi z_{m}^{\sigma} z_{n}^{\sigma^{\prime}} .
$$

Each coefficient $F^{\sigma, \sigma^{\prime}}\left(\mathrm{s}(m), \sigma m+\sigma^{\prime} n\right) \circ \Phi$ depends on $n, m, \sigma, \sigma^{\prime}$ only through $\mathbf{s}(m)$, $\sigma m+\sigma^{\prime} n, \sigma, \sigma^{\prime}$. Hence, in order to conclude that $F \circ \Phi \in \mathcal{T}_{s, 2 r}\left(N, \theta, \mu^{\prime}\right)$ it remains only to prove that $F^{\sigma, \sigma^{\prime}}\left(\mathbf{s}(m), \sigma m+\sigma^{\prime} n\right) \circ \Phi \in \mathcal{L}_{s, 2 r}\left(N, \mu^{\prime}, \sigma m+\sigma^{\prime} n\right)$, see Definition 3.1. Each monomial $\mathfrak{m}_{\alpha, \beta}$ of $F^{\sigma, \sigma^{\prime}}\left(\mathbf{s}(m), \sigma m+\sigma^{\prime} n\right) \in \mathcal{L}_{R / 2}\left(N, \mu^{\prime}, \sigma m+\sigma^{\prime} n\right)$ satisfies

$$
\sum_{l=1}^{n}\left(\alpha_{\mathfrak{j}_{l}}+\beta_{\mathfrak{j}_{l}}\right)\left|\mathfrak{j}_{l}\right|+\sum_{j \in \mathbb{Z} \backslash \mathcal{I}}\left(\alpha_{j}+\beta_{j}\right)|j|<\mu^{\prime} N^{L} \quad \text { and } \quad \mathfrak{p}\left(\mathfrak{m}_{\alpha, \beta}\right)<N^{b}
$$

by the hypothesis $\Pi_{\mathfrak{p} \geq N^{b}} F=0$. Hence $\mathfrak{m}_{\alpha, \beta} \circ \Phi$ (see (7.71)) is $\left(N, \mu^{\prime}\right)$-low momentum, in particular $\left|\alpha^{(1)}-\beta^{(1)}\right| \leq \mathfrak{p}\left(\mathfrak{m}_{\alpha, \beta}\right)<N^{b}$.

Proof of Proposition 7.2. - Since $F \in \mathcal{Q}_{R / 2}^{T}\left(N_{0}, \theta, \mu^{\prime}\right)$ (see Definition 3.4), for all $N \geq N_{0}$, there is a Töplitz approximation $\tilde{F} \in \mathcal{T}_{R / 2}\left(N, \theta, \mu^{\prime}\right)$ of $F$, namely

$$
\Pi_{N, \theta, \mu^{\prime}} F=\tilde{F}+N^{-1} \hat{F} \quad \text { with } \quad\left\|X_{F}\right\|_{R / 2},\left\|X_{\tilde{F}}\right\|_{R / 2},\left\|X_{\hat{F}}\right\|_{R / 2}<2\|F\|_{R / 2, N_{0}, \theta, \mu^{\prime}}^{T} .
$$

In order to prove that $f:=F \circ \Phi \in \mathcal{Q}_{s, r}^{T}\left(N_{0}, \theta, \mu\right)$ we define its candidate Töplitz approximation

$$
\tilde{f}:=\Pi_{N, \theta, \mu}\left(\left(\Pi_{\mathfrak{p}<N^{b}} \tilde{F}\right) \circ \Phi\right),
$$

see (7.66). Lemma 7.10 applied to $\Pi_{\mathfrak{p}<N^{b}} \tilde{F} \in \mathcal{T}_{R / 2}\left(N, \theta, \mu^{\prime}\right)$ implies that $\left(\Pi_{\mathfrak{p}<N^{b}} \tilde{F}\right) \circ \Phi \in \mathcal{T}_{s, 2 r}\left(N, \theta, \mu^{\prime}\right)$ and then, applying the projection $\Pi_{N, \theta, \mu}$ we get $\tilde{f} \in \mathcal{T}_{s, 2 r}(N, \theta, \mu) \subset \mathcal{T}_{s, r}(N, \theta, \mu)$. Moreover, by (7.74) and applying Lemma 7.7 to $\Pi_{\mathfrak{p}<N^{b}} \tilde{F}$ (note that $\Pi_{\mathfrak{p}<N^{b}} \tilde{F}$ is either zero or it is in $\mathcal{H}_{R / 2}^{d}$ with $d \geq 2$ because it is bilinear), we get

$$
\begin{aligned}
& \left\|X_{\tilde{f}}\right\|_{s, r, \mathcal{O}_{\rho}} \stackrel{(2.80)}{\leq}\left\|X_{\left.\left(\Pi_{\mathfrak{p}<N^{b}} \tilde{F}\right) \circ \Phi\right)}\right\|_{s, r, \mathcal{O}_{\rho}} \stackrel{(7.54)}{\leftarrow} \quad(8 r / R)^{d-2}\left\|X_{\Pi_{\mathfrak{p}<N^{b}} \tilde{F}}\right\|_{R / 2} \\
& \stackrel{\text { (2.80),(7.73) }}{\lessdot}(8 r / R)^{d-2}\|F\|_{R / 2, N_{0}, \theta, \mu^{\prime}}^{T} \text {. }
\end{aligned}
$$

Moreover the Töplitz defect is

$$
\begin{array}{rll}
\hat{f} & := & N\left(\Pi_{N, \theta, \mu} f-\tilde{f}\right) \stackrel{(7.74)}{=} N \Pi_{N, \theta, \mu}\left(\left(F-\Pi_{\mathfrak{p}<N^{b}} \tilde{F}\right) \circ \Phi\right) \\
& = & N \Pi_{N, \theta, \mu}((F-\tilde{F}) \circ \Phi)+N \Pi_{N, \theta, \mu}\left(\left(\tilde{F}-\Pi_{\mathfrak{p}<N^{b}} \tilde{F}\right) \circ \Phi\right) \\
\stackrel{(7.73),(7.66)}{=} & \Pi_{N, \theta, \mu}(\hat{F} \circ \Phi)+N \Pi_{N, \theta, \mu}\left(\left(F-\Pi_{N, \theta, \mu^{\prime}} F\right) \circ \Phi\right)+N \Pi_{N, \theta, \mu}\left(\left(\Pi_{\mathfrak{p} \geq N^{b}} \tilde{F}\right) \circ \Phi\right) \\
& \stackrel{(7.69)}{=} & \Pi_{N, \theta, \mu}(\hat{F} \circ \Phi)+N f^{*}+N \Pi_{N, \theta, \mu}\left(\left(\Pi_{\mathfrak{p} \geq N^{b}} \tilde{F}\right) \circ \Phi\right) .
\end{array}
$$


Using (2.80), Lemmata 7.8 and 7.9 imply that, since $N 2^{-\frac{N^{b}}{2 \kappa}+1} \leq 1, \forall N \geq N_{0}$ by (7.47),

$$
\begin{aligned}
& \left\|X_{\hat{f}}\right\|_{s, r, \mathcal{O}_{\rho}} \leq\left\|X_{\hat{F} \circ \Phi}\right\|_{s, r, \mathcal{O}_{\rho}}+N 2^{-\frac{N^{b}}{2 \kappa}+1}\left(\left\|X_{F \circ \Phi}\right\|_{s, 2 r, \mathcal{O}_{2 \rho}}+\left\|X_{\tilde{F} \circ \Phi}\right\|_{s, 2 r, \mathcal{O}_{2 \rho}}\right) \\
& \lessdot\left\|X_{\hat{F} \circ \Phi}\right\|_{s, 2 r, \mathcal{O}_{\rho}}+\left\|X_{F \circ \Phi}\right\|_{s, 2 r, \mathcal{O}_{2 \rho}}+\left\|X_{\tilde{F} \circ \Phi}\right\|_{s, 2 r, \mathcal{O}_{2 \rho}} \\
& \stackrel{(7.54)}{\leftarrow}(8 r / R)^{d-2}\left(\left\|X_{\hat{F}}\right\|_{R / 2}+\left\|X_{F}\right\|_{R / 2}+\left\|X_{\tilde{F}}\right\|_{R / 2}\right) \\
& \stackrel{(7.73)}{\lessdot}(8 r / R)^{d-2}\|F\|_{R / 2, N_{0}, \theta, \mu^{\prime}}^{T}
\end{aligned}
$$

(to get (7.76) we also note that $F, \hat{F}, \tilde{F} \in \mathcal{H}_{R / 2}^{d}$ with $d=0,1$, unless they are zero).

The bound (7.48) follows by (7.54), (7.75), (7.77).

We conclude this subsection with a lemma, similar to Lemma 7.7, used in Lemma 7.12 (see (7.91)).

Lemma 7.11. - Let $F \in \mathcal{H}_{R / 2}, f:=F \circ \Phi$ and $\tilde{f}(x, y):=f(x, y, 0,0)-f(x, 0,0,0)$. Then, assuming (7.42),

$$
\left\|X_{\tilde{f}}\right\|_{s, 2 r, \mathcal{O}_{\rho} \cup \mathcal{O}_{2 \rho}} \lessdot\left\|X_{F}\right\|_{R / 2} .
$$

Moreover if $F$ preserves momentum then so does $\tilde{f}$.

Proof. - We proceed as in Lemma 7.7. The main difference is that here there are no $(z, \bar{z})$-variables and the sum in (7.56) runs over $i \neq 0$. Then in the product in (7.57) (at least) one of the sums is on $i_{l} \geq 1$. Therefore we can use the second estimate in (7.52) gaining a factor $^{(3)} 8 r^{2} / \rho$ (since $\left|y_{l}\right| /\left|\xi_{l}\right| \leq 8 r^{2} / \rho$ by (7.41)). Continuing as in the proof of Lemma 7.7 we get (recall (7.54) with $d=0$ )

$$
\left\|X_{\tilde{f}}\right\|_{s, 2 r, \mathcal{O}_{\rho} \cup \mathcal{O}_{2 \rho}} \lessdot\left(r^{2} / \rho\right)(r / R)^{-2}\left\|X_{F}\right\|_{R / 2} \stackrel{(7.42)}{\leftarrow}\left\|X_{F}\right\|_{R / 2}
$$

proving (7.78).

\subsection{Proof of Theorem 1.1}

We now introduce the action-angle variables (7.40) (via the map (7.39)) in the Birkhoff normal form Hamiltonian (7.8). Hence we obtain the parameter dependent family of Hamiltonians

$$
H^{\prime}:=H_{\text {Birkhoff }} \circ \Phi=\mathcal{N}+P
$$

where (up to a constant), by (7.6),

$$
\begin{gathered}
\mathcal{N}:=\omega(\xi) \cdot y+\Omega(\xi) z \bar{z}, \quad P:=\frac{1}{2} A y \cdot y+B y \cdot z \bar{z}+\hat{G}(z, \bar{z})+K^{\prime}(x, y, z, \bar{z} ; \xi) \\
\omega(\xi):=\bar{\omega}+A \xi, \bar{\omega}:=\left(\lambda_{j_{1}}, \ldots, \lambda_{j_{n}}\right), \quad \Omega(\xi):=\bar{\Omega}+B \xi, \bar{\Omega}:=\left(\lambda_{j}\right)_{j \in \mathbb{Z} \backslash \mathcal{I}}
\end{gathered}
$$

$A=\left(A_{l h}\right)_{1 \leq l, h \leq n}, A_{l h}:=12\left(2-\delta_{l h}\right), B=\left(B_{j l}\right)_{j \in \mathbb{Z} \backslash \mathcal{I}, 1 \leq l \leq n}, B_{j l}:=24, K^{\prime}:=K \circ \Phi$.

(3) Actually we have the constant 3 instead of 2 in (7.58) and $3 e^{s}$ instead of $2 e^{s}$ in (7.60) and (7.62). 
The parameters $\xi$ stay in the set $\mathcal{O}_{\rho}$ defined in (7.41) with $\rho=C_{*} R^{2}$ as in (7.42). As in (4.6) we decompose the perturbation

$$
P=P_{00}+\bar{P} \quad \text { where } \quad P_{00}(x ; \xi):=K^{\prime}(x, 0,0,0 ; \xi), \quad \bar{P}:=P-P_{00} .
$$

Lemma 7.12. - Let $s, r>0$ as in (7.42) and $N$ large enough (w.r.t. $\mathrm{m}, \mathcal{I}, L, b)$. Then

$$
\left\|X_{P_{00}}\right\|_{s, r} \lessdot R^{6} r^{-2}, \quad\|\bar{P}\|_{s, r, N, 2,2}^{T} \lessdot r^{2}+R^{5} r^{-1}
$$

and, for $\lambda>0$,

$$
\left|X_{P_{00}}\right|_{s, r}^{\lambda} \lessdot(1+\lambda / \rho) R^{6} r^{-2}, \quad\left|X_{\bar{P}}\right|_{s, r}^{\lambda} \lessdot(1+\lambda / \rho)\left(r^{2}+R^{5} r^{-1}\right),
$$

for $\xi$ belonging to

$$
\mathcal{O}(\rho):=\left\{\xi \in \mathbb{R}^{n}: \frac{2}{3} \rho \leq \xi_{l} \leq \frac{3}{4} \rho, l=1, \ldots, n\right\} \subset \mathcal{O}_{\rho} .
$$

Proof. - By the Definition (7.83) we have

$$
\begin{aligned}
& \left\|X_{P_{00}}\right\|_{s, r} \stackrel{(2.55)}{\leq}\left\|X_{K^{\prime}}\right\|_{s, r} \stackrel{(3.32)}{\leq}\left\|K^{\prime}\right\|_{s, r, N, 2,2}^{T} \stackrel{(7.82)}{=}\|K \circ \Phi\|_{s, r, N, 2,2}^{T} \\
& \stackrel{(7.48)}{\leftarrow}\left(\frac{r}{R}\right)^{-2}\|K\|_{R / 2, N, 2,2}^{T}
\end{aligned}
$$

(applying (7.48) with $d \rightsquigarrow 0, N_{0} \rightsquigarrow N, \theta \rightsquigarrow 2, \mu \rightsquigarrow 2, \mu^{\prime} \rightsquigarrow 3$ ) and taking $N$ large enough so that (7.47) holds. Take also $N \geq N_{0}^{\prime}$ defined in Proposition 7.1. Then by (7.87) we get

$$
\left\|X_{P_{00}}\right\|_{s, r} \stackrel{(3.35)}{\leftarrow}\left(\frac{r}{R}\right)^{-2}\|K\|_{R / 2, N_{0}^{\prime}, 2,2}^{T} \stackrel{(7.10)}{\leftarrow}\left(\frac{r}{R}\right)^{-2} R^{4} \lessdot \frac{R^{6}}{r^{2}}
$$

proving the first estimate in (7.84). Let us prove the second bound. By (7.83) and (7.80) we write

$$
\bar{P}=\frac{1}{2} A y \cdot y+B y \cdot z \bar{z}+\hat{G}(z, \bar{z})+K_{1}+K_{2}
$$

where

$$
K_{1}:=K^{\prime}(x, y, z, \bar{z} ; \xi)-K^{\prime}(x, y, 0,0 ; \xi), \quad K_{2}:=K^{\prime}(x, y, 0,0 ; \xi)-K^{\prime}(x, 0,0,0 ; \xi) .
$$

Using (7.7) (note that $r<R$ by (7.42)) for $N \geq N_{0}$ large enough to fulfill (3.1), we have by (3.35)

$$
\left\|\frac{1}{2} A y \cdot y+B y \cdot z \bar{z}+\hat{G}(z, \bar{z})\right\|_{s, r, N, 2,2}^{T} \lessdot r^{2} .
$$

By (7.48) (with $d \rightsquigarrow 1, N_{0} \rightsquigarrow N, \mu \rightsquigarrow 2, \mu^{\prime} \rightsquigarrow 3$ ), for $N \geq N_{0}(\mathrm{~m}, \mathcal{I}, L, b)$ large enough, we get

$$
\left\|K_{1}\right\|_{s, r, N, 2,2}^{T} \lessdot\left(\frac{r}{R}\right)^{-1} R^{4} \lessdot \frac{R^{5}}{r} .
$$

Moreover, since $K_{2}$ does not depend on $(z, \bar{z})$, we have

$$
\left\|K_{2}\right\|_{s, r, N, 2,2}^{T} \stackrel{(3.34)}{=}\left\|X_{K_{2}}\right\|_{s, r} \stackrel{(7.78)}{\leftarrow}\left\|X_{K}\right\|_{R / 2} \stackrel{(3.32)}{\leftarrow}\|K\|_{R / 2, N_{0}^{\prime}, 2,3}^{T} \stackrel{(7.10)}{\leftarrow} R^{4} .
$$

In conclusion, (7.88), (7.89), (7.90), (7.91) imply the second estimate in (7.84):

$$
\|\bar{P}\|_{s, r, N, 2,2}^{T} \lessdot r^{2}+\frac{R^{5}}{r}+R^{4} \stackrel{(7.42)}{\lessdot} r^{2}+\frac{R^{5}}{r} .
$$


Let us prove the estimates (7.85) for the Lipschitz norm defined in (2.88) (which involves only the sup-norm of the vector fields). First

$$
\left|X_{P_{00}}\right|_{s, r} \stackrel{(2.62)}{\leq}\left\|X_{P_{00}}\right\|_{s, r} \stackrel{(7.84)}{\leftarrow} R^{6} r^{-2},\left|X_{\bar{P}}\right|_{s, r} \stackrel{(2.62)}{\leq}\left\|X_{\bar{P}}\right\|_{s, r} \stackrel{(3.32)}{\leq}\|\bar{P}\|_{s, r, N, 2,2}^{T} \stackrel{(7.84)}{\leftarrow} r^{2}+R^{5} r^{-1} .
$$

Next, since the vector fields $X_{P_{00}}, X_{\bar{P}}$ are analytic in the parameters $\xi \in \mathcal{O}_{\rho}$, Cauchy estimates in the domain $\mathcal{O}(\rho) \subset \mathcal{O}_{\rho}$ (see (7.86)) imply

$$
\left|X_{P_{00}}\right|_{s, r, \mathcal{O}(\rho)}^{\operatorname{lip}} \lessdot \rho^{-1}\left|X_{P_{00}}\right|_{s, r, \mathcal{O}_{\rho}} \lessdot R^{6} r^{-2}, \quad\left|X_{\bar{P}}\right|_{s, r, \mathcal{O}(\rho)}^{\operatorname{lip}} \lessdot \rho^{-1}\left|X_{\bar{P}}\right|_{s, r, \mathcal{O}_{\rho}} \lessdot r^{2}+R^{5} r^{-1}
$$

and (7.85) are proved.

All the assumptions of Theorems 4.1-4.2 are fulfilled by $H^{\prime}$ in (7.79) with parameters $\xi \in \mathcal{O}(\rho)$ defined in (7.86). Note that the sets $\mathcal{O}=[\rho / 2, \rho]^{n}$ defined in Theorem 4.2 and $\mathcal{O}(\rho)$ defined in (7.86) are diffeomorphic through $\xi_{i} \mapsto\left(7 \rho+2 \xi_{i}\right) / 12$. The hypotheses (A1)(A2) follow from (7.81), (7.82) with

$$
a(\xi)=24 \sum_{l=1, \ldots, n} \xi_{l}, \quad \text { and } \quad M_{0}=24+\|A\| .
$$

Then (A3)-(A4) and the quantitative bound (4.7) follow by (7.84)-(7.85), choosing

$s=1, r=R^{1+\frac{3}{4}}, \rho=C_{*} R^{2}$ as in (7.42), $N$ as in Lemma 7.12, $\theta=2, \mu=2, \gamma=R^{3+\frac{1}{5}}$, and taking $R$ small enough. Hence Theorem 4.1 applies.

Let us verify that also the assumptions of Theorem 4.2 are fulfilled. Indeed (4.13) follows by (7.81), (7.82) with $\vec{a}=24(1, \ldots, 1) \in \mathbb{R}^{n}$. The matrix $A$ defined in (7.82) is invertible and

$$
A^{-1}=\left(A_{l h}^{-1}\right)_{1 \leq l, h \leq n}, \quad A_{l h}^{-1}=\frac{1}{12}\left(\frac{2}{2 n-1}-\delta_{l h}\right) .
$$

Finally the non-degeneracy assumption (4.14) is satisfied because $A=A^{T}$ and

$$
2 A^{-1} \vec{a}=\frac{4}{2 n-1}(1, \ldots, 1) \notin \mathbb{Z}^{n} \backslash 0 .
$$

We deduce that the Cantor set of parameters $\mathcal{O}_{\infty} \subset \mathcal{O}$ in (4.11) has asymptotically full density because

$$
\frac{\left|\mathcal{O} \backslash \mathcal{O}_{\infty}\right|}{|\mathcal{O}|} \stackrel{(4.16)}{\leftarrow} \rho^{-1} \gamma^{2 / 3} \stackrel{(7.92)}{\leftarrow} R^{-2} R^{\frac{2}{3}\left(3+\frac{1}{5}\right)}=R^{\frac{2}{15}} \rightarrow 0 .
$$

The proof of Theorem 1.1 is now completed.

REMARK 7.1. - The terms $\sum_{k \geq 5} f_{k} s^{k}$ in (1.2) contribute to the Hamiltonian (7.1) with monomials of order 6 or more and (7.8) holds (with a possibly different $K$ satisfying (7.10)). On the contrary, the term $f_{4} s^{4}$ in (1.2) would add monomials of order 5 to the Hamiltonian in (7.1). Hence (7.10) holds with $R^{3}$ instead of $R^{4}$. This estimate is not sufficient. These 5 -th order terms should be removed by a Birkhoff normal form. For simplicity, we did not pursue this point. 


\section{Appendix}

Proof of Lemma 2.14. - We need some notation: we write $E=\oplus_{j=1}^{4} E_{j}, E_{1}:=\left(\mathbb{C}^{n},||_{\infty}\right)$, $E_{2}:=\left(\mathbb{C}^{n},||_{1}\right), E_{3}:=E_{4}:=\ell_{\mathcal{I}}^{a, p}$ so that a vector $v=(x, y, z, \bar{z}) \in E$ can be expressed by its four components $v^{(j)} \in E_{j}, v^{(1)}:=x, v^{(2)}:=y, v^{(3)}:=z, v^{(4)}:=\bar{z}$, and the norm (2.2) is

$$
\|v\|_{E, s, r}:=\sum_{j=1}^{4} \frac{\left|v^{(j)}\right|_{E_{j}}}{\rho_{j}}, \quad \text { where } \rho_{1}=s, \quad \rho_{2}=r^{2}, \quad \rho_{3}=\rho_{4}=r .
$$

We are now ready to prove (2.65). By definition

$$
\begin{aligned}
\|d X(v)\|_{\mathcal{L}\left((E, s, r) ;\left(E, s^{\prime}, r^{\prime}\right) \xi\right.} & =\sup _{\|Y\|_{E, s, r} \leq 1}\|d X(v)[Y]\|_{E, s^{\prime}, r^{\prime}} \stackrel{(8.1)}{=} \sup _{\|Y\|_{E, s, r} \leq 1} \sum_{i=1}^{4} \frac{\left|d X^{(i)}(v)[Y]\right|_{E_{i}}}{\rho_{i}^{\prime}} \\
& =\sup _{\|Y\|_{E, s, r} \leq 1} \sum_{i=1}^{4} \frac{\left|\sum_{j=1}^{4} d_{v^{(j)}} X^{(i)}(v) Y^{(j)}\right|_{E_{i}}}{\rho_{i}^{\prime}} \\
& \leq \sup _{\|Y\|_{E, s, r} \leq 1} \sum_{i, j=1}^{4} \frac{\left|d_{v^{(j)}} X^{(i)}(v) Y^{(j)}\right|_{E_{i}}}{\rho_{i}^{\prime}} \\
& \leq \sup _{\|Y\|_{E, s, r} \leq 1} \sum_{i, j=1}^{4} \frac{1}{\rho_{i}^{\prime}}\left\|d_{v^{(j)}} X^{(i)}(v)\right\|_{\mathcal{L}\left(E_{j}, E_{i}\right)}\left|Y^{(j)}\right|_{E_{j}} \\
& \leq \sup _{\|Y\|_{E, s, r} \leq 1} \sup _{\tilde{v} \in D(s, r)} \sum_{i, j=1}^{4} \frac{1}{\rho_{i}^{\prime}} \frac{\left|X^{(i)}(\tilde{v})\right|_{E_{i}}}{\left(\rho_{j}-\rho_{j}^{\prime}\right)}\left|Y^{(j)}\right|_{E_{j}}
\end{aligned}
$$

by the Cauchy estimates in Banach spaces. Then

$$
\begin{aligned}
\|d X(v)\|_{\mathcal{L}\left((E, s, r) ;\left(E, s^{\prime}, r^{\prime}\right)\right)} & \leq \sup _{\tilde{v} \in D(s, r)} \sum_{i=1}^{4} \frac{\rho_{i}}{\rho_{i}^{\prime}} \frac{\left|X^{(i)}(\tilde{v})\right|_{E_{i}}}{\rho_{i}} \sup _{\|Y\|_{E, s, r} \leq 1} \sum_{j=1}^{4}\left(1-\frac{\rho_{j}^{\prime}}{\rho_{j}}\right)^{-1} \frac{\left|Y^{(j)}\right|_{E_{j}}}{\rho_{j}} \\
& \stackrel{(8.1)}{\leq} \max _{i=1, \ldots, 4} \frac{\rho_{i}}{\rho_{i}^{\prime}} \max _{j=1, \ldots, 4}\left(1-\frac{\rho_{j}^{\prime}}{\rho_{j}}\right)^{-1} \sup _{\tilde{v} \in D(s, r)}\|X(\tilde{v})\|_{E, s, r} \leq 4 \delta^{-1}|X|_{s, r}
\end{aligned}
$$

by (2.53), (2.66). This proves (2.65).

Proof of Lemma 7.2. - We first extend Lemma 4 of [28] proving that:

Lemma 8.1. - If $0 \leq i \leq j \leq k \leq l$ with $i \pm j \pm k \pm l=0$ for some combination of plus and minus signs and $(i, j, k, l) \neq(p, p, q, q)$ for $p, q \in \mathbb{N}$, then, there exists an absolute constant $c>0$, such that

$$
\left| \pm \lambda_{i}(\mathrm{~m}) \pm \lambda_{j}(\mathrm{~m}) \pm \lambda_{k}(\mathrm{~m}) \pm \lambda_{l}(\mathrm{~m})\right| \geq c \mathrm{~m}\left(i^{2}+\mathrm{m}\right)^{-3 / 2}
$$

for ALL possible combinations of plus and minus signs.

Proof. - When $i>0$ it is a reformulation of the statement of Lemma 4 of [28]. Let us prove it also for $i=0$. Then $j \pm k \pm l=0$ for some combination of plus and minus signs. Since $(i, j, k, l) \neq(0,0, q, q)$, the only possibility is $l=j+k$ with $j \geq 1$ (otherwise $i=j=0$ and $k=l$ ). We have to study

$$
\delta(\mathrm{m}):= \pm \lambda_{0}(\mathrm{~m}) \pm \lambda_{j}(\mathrm{~m}) \pm \lambda_{k}(\mathrm{~m}) \pm \lambda_{l}(\mathrm{~m})
$$


for all possible combinations of plus and minus signs. To this end, we distinguish them according to their number of plus and minus signs. To shorten notation we let, for example, $\delta_{++-+}=\lambda_{0}+\lambda_{j}-\lambda_{k}+\lambda_{l}$, similarly for the other combinations. The only interesting cases are when there are one or two minus signs. The case when there are no (or four) minus signs is trivial. When there are 3 minus signs we reduce to the case with one minus sign by a global sign change.

One minus sign. Since $\delta_{++-+}, \delta_{+-++}, \delta_{-+++} \geq \delta_{+++-}:=\delta$ we study only the last case. We have

$$
\delta(0)=j+k-l=0, \quad \delta^{\prime}(\mathrm{m})=\frac{1}{2}\left(\frac{1}{\lambda_{0}}+\frac{1}{\lambda_{j}}+\frac{1}{\lambda_{k}}-\frac{1}{\lambda_{l}}\right) \geq \frac{1}{2 \lambda_{0}}=\frac{1}{2 \sqrt{\mathrm{m}}} .
$$

Therefore $\delta(\mathrm{m}) \geq \sqrt{\mathrm{m}} \geq \mathrm{cm}(1+\mathrm{m})^{-3 / 2}$ for an absolute constant $c>0$.

Two minus signs. Now we have $\delta_{-+-+}, \delta_{--++} \geq \delta_{+--+}$and all other cases reduce to these ones by inverting signs. So we consider only $\delta=\delta_{+--+}$. Since the function $f(t):=\sqrt{t^{2}+\mathrm{m}}$ is monotone increasing and convex for $t \geq 0$, we have the estimate

$$
\lambda_{l}-\lambda_{k} \geq \lambda_{l-p}-\lambda_{k-p}, \quad \forall 0 \leq p \leq k .
$$

Hence $\lambda_{l}-\lambda_{k} \geq \lambda_{j+1}-\lambda_{1}$ and $\lambda_{j+1}-\lambda_{j} \geq \lambda_{2}-\lambda_{1}$ (using $j=l-k \geq 1$ ). Therefore

$$
\delta=\lambda_{0}-\lambda_{j}-\lambda_{k}+\lambda_{l} \geq \lambda_{0}-\lambda_{j}-\lambda_{1}+\lambda_{j+1} \geq \lambda_{2}-2 \lambda_{1}+\lambda_{0} \geq \mathrm{m}(4+\mathrm{m})^{-3 / 2} .
$$

The last inequality follows since $f^{\prime \prime}(t)=\mathrm{m}\left(t^{2}+\mathrm{m}\right)^{-3 / 2}$ is decreasing and $\lambda_{2}-2 \lambda_{1}+\lambda_{0}=$ $f(2)-2 f(1)+f(0)=f^{\prime \prime}(\xi) \geq f^{\prime \prime}(2)$ for some $\xi \in(0,2)$.

We complete the proof of Lemma 7.2. We first consider the trivial cases (7.12)-(7.14).

CASE (7.12). Since $\sum_{i} \sigma_{i} \neq 0$ is even, (7.16) follows by

$$
\left|\sigma \cdot \lambda_{\vec{\jmath}}\right|=\left|\sum_{i} \sigma_{i} \lambda_{\overrightarrow{0}}\right| \geq 2 \lambda_{\overrightarrow{0}}=2 \sqrt{\mathrm{m}} \geq \mathrm{m}(1+\mathrm{m})^{-3 / 2} .
$$

CASE (7.13). By $\vec{\sigma} \cdot \vec{\jmath}=\left(\sigma_{3}+\sigma_{4}\right) q=0, q \neq 0$, we deduce $\sigma_{3}=-\sigma_{4}$. Hence (7.16) follows by

$$
\left|\sigma \cdot \lambda_{\vec{\jmath}}\right|=\left|\left(\sigma_{1}+\sigma_{2}\right) \lambda_{0}\right|=2 \sqrt{\mathrm{m}} \geq \mathrm{m}(1+\mathrm{m})^{-3 / 2} .
$$

CASE (7.14). Since $\vec{\jmath}=(p, p,-p,-p)$ and $\sigma_{1}=\sigma_{2}$ then $\vec{\sigma} \cdot \vec{\jmath}=0$ implies $\sigma_{3}=\sigma_{4}=\sigma_{2}$ and

$$
\left|\sigma \cdot \lambda_{\vec{\jmath}}\right|=\left|4 \lambda_{p}\right|=4 \sqrt{p^{2}+\mathrm{m}} \geq \mathrm{m}\left(p^{2}+\mathrm{m}\right)^{-3 / 2} .
$$

CASE (7.15). Set $\left|j_{1}\right|=: i,\left|j_{2}\right|=: j,\left|j_{3}\right|=: k,\left|j_{4}\right|=: l$. After reordering we can assume $0 \leq i \leq j \leq k \leq l$. Since, by assumption, $\vec{\sigma} \cdot \vec{\jmath}=0$, the following combination of plus and minus signs gives $\mathbf{s}\left(j_{1}\right) \sigma_{1} i+\mathbf{s}\left(j_{2}\right) \sigma_{2} j+\mathbf{s}\left(j_{3}\right) \sigma_{3} k+\mathbf{s}\left(j_{4}\right) \sigma_{4} l=0$. Hence Lemma 8.1 implies (7.16) for every $\vec{\jmath}$ except when $\left|j_{1}\right|=\left|j_{2}\right|$ and $\left|j_{3}\right|=\left|j_{4}\right|$ (in this case $i=j$ and $k=l$ and Lemma 8.1 does not apply). We now prove that (7.16) holds also in these cases. We have that $\vec{\sigma} \cdot \lambda_{\vec{\jmath}}=\left(\sigma_{1}+\sigma_{2}\right) \lambda_{j_{1}}+\left(\sigma_{3}+\sigma_{4}\right) \lambda_{j_{3}}$ where $\sigma_{a}+\sigma_{b}=0, \pm 2$ so that (7.16) holds trivially unless $\sigma_{1}+\sigma_{2}=-\left(\sigma_{3}+\sigma_{4}\right)$. We consider this last case. If $\sigma_{1}+\sigma_{2}=-\left(\sigma_{3}+\sigma_{4}\right)=0$ then the equality $\vec{\sigma} \cdot \vec{\jmath}=\sigma_{1}\left(j_{1}-j_{2}\right)+\sigma_{3}\left(j_{3}-j_{4}\right)=0$ implies that $j_{1}, \ldots, j_{4}$ are pairwise equal, contrary to our hypothesis. If $\sigma_{1}+\sigma_{2}= \pm 2$ and $i:=\left|j_{1}\right|<k:=\left|j_{3}\right|$ then

$$
\left|\vec{\sigma} \cdot \lambda_{\vec{\jmath}}\right| \geq 2 \lambda_{j_{3}}-2 \lambda_{j_{1}}=2 \lambda_{k}-2 \lambda_{i} \stackrel{(8.3)}{\geq} 2 \lambda_{k-i}-2 \lambda_{0} \stackrel{(k>i)}{\geq} 2 \lambda_{1}-2 \lambda_{0} \geq 1 / \sqrt{1+\mathrm{m}}
$$


giving (7.16). If $\left|j_{1}\right|=\left|j_{2}\right|=\left|j_{3}\right|=\left|j_{4}\right|$ and $\sigma_{1}+\sigma_{2}=-\left(\sigma_{3}+\sigma_{4}\right)= \pm 2$ then the relation $\vec{\sigma} \cdot \vec{\jmath}=\sigma_{1}\left(j_{1}+j_{2}-j_{3}-j_{4}\right)=0$ implies that the $j_{1}, \ldots, j_{4}$ are pairwise equal, contrary to the hypothesis.

\subsection{Acknowledgments}

This research was supported by the European Research Council under FP7 "New Connections between dynamical systems and Hamiltonian PDEs with small divisors phenomena" and partially by the PRIN2009 grant "Critical Point Theory and Perturbative Methods for Nonlinear Differential Equations".

We thank Benoît Grébert for pointing out a technical mistake in the previous version.

\section{REFERENCES}

[1] D. BAmbusi, B. GRÉBERT, Birkhoff normal form for partial differential equations with tame modulus, Duke Math. J. 135 (2006), 507-567.

[2] M. Berti, L. Biasco, Branching of Cantor manifolds of elliptic tori and applications to PDEs, Comm. Math. Phys. 305 (2011), 741-796.

[3] M. Berti, P. Bolle, Sobolev quasi-periodic solutions of multidimensional wave equations with a multiplicative potential, Nonlinearity 25 (2012), 2579-2613.

[4] M. Berti, P. Bolle, Quasi-periodic solutions with Sobolev regularity of NLS on $T^{d}$ with a multiplicative potential, Eur. Jour. Math. 15 (2013), 229-286.

[5] J. Bourgain, Construction of quasi-periodic solutions for Hamiltonian perturbations of linear equations and applications to nonlinear PDE, Int. Math. Res. Not. 1994 (1994).

[6] J. Bourgain, Quasi-periodic solutions of Hamiltonian perturbations of 2D linear Schrödinger equations, Ann. of Math. 148 (1998), 363-439.

[7] J. Bourgain, Periodic solutions of nonlinear wave equations, in Harmonic analysis and partial differential equations (Chicago, IL, 1996), Chicago Lectures in Math., Univ. Chicago Press, 1999, 69-97.

[8] J. Bourgain, Green's function estimates for lattice Schrödinger operators and applications, Annals of Math. Studies 158, Princeton Univ. Press, 2005. 
[9] L. Chierchia, J. You, KAM tori for 1D nonlinear wave equations with periodic boundary conditions, Comm. Math. Phys. 211 (2000), 497-525.

[10] W. Craig, Problèmes de petits diviseurs dans les équations aux dérivées partielles, Panoramas et Synthèses 9, Soc. Math. France, 2000.

[11] W. Craig, C. E. Wayne, Newton's method and periodic solutions of nonlinear wave equations, Comm. Pure Appl. Math. 46 (1993), 1409-1498.

[12] J.-M. Delort, A quasi-linear Birkhoff normal forms method. Application to the quasi-linear Klein-Gordon equation on $\mathbb{S}^{1}$, Astérisque 341 (2012).

[13] J.-M. Delort, J. Szeftel, Long-time existence for small data nonlinear KleinGordon equations on tori and spheres, Int. Math. Res. Not. 2004 (2004), 1897-1966.

[14] L. H. Eliasson, S. B. Kuksin, KAM for the nonlinear Schrödinger equation, Ann. of Math. 172 (2010), 371-435.

[15] J. Geng, X. Xu, J. You, An infinite dimensional KAM theorem and its application to the two dimensional cubic Schrödinger equation, Adv. Math. 226 (2011), 5361-5402.

[16] J. Geng, J. You, A KAM theorem for Hamiltonian partial differential equations in higher dimensional spaces, Comm. Math. Phys. 262 (2006), 343-372.

[17] P. GÉrard, S. Grellier, Effective integrable dynamics for some nonlinear wave equation, preprint http://hal archives-ouvertes.fr/hal-00635686/fr/.

[18] B. Grébert, L. Thomann, KAM for the quantum harmonic oscillator, Comm. Math. Phys. 307 (2011), 383-427.

[19] T. Kappeler, J. Pöschel, KAM and KdV, Springer, 2003.

[20] S. B. Kuksin, Hamiltonian perturbations of infinite-dimensional linear systems with imaginary spectrum, Funktsional. Anal. i Prilozhen. 21 (1987), 22-37, 95.

[21] S. B. Kuksin, A KAM-theorem for equations of the Korteweg-de Vries type, Rev. Math. Math. Phys. 10 (1998), 1-64.

[22] S. B. Kuksin, Analysis of Hamiltonian PDEs, Oxford Lecture Series in Mathematics and its Applications 19, Oxford Univ. Press, 2000.

[23] S. Kuksin, J. Pöschel, Invariant Cantor manifolds of quasi-periodic oscillations for a nonlinear Schrödinger equation, Ann. of Math. 143 (1996), 149-179.

[24] J. LiU, X. YUAN, A KAM theorem for Hamiltonian partial differential equations with unbounded perturbations, Comm. Math. Phys. 307 (2011), 629-673.

[25] N. V. Nikolenko, The method of Poincaré normal forms in problems of integrability of equations of evolution type, Uspekhi Mat. Nauk 41 (1986), 109-152, 263.

[26] J. PösChel, On elliptic lower-dimensional tori in Hamiltonian systems, Math. Z. 202 (1989), 559-608.

[27] J. Pöschel, A KAM-theorem for some nonlinear partial differential equations, Ann. Scuola Norm. Sup. Pisa Cl. Sci. 23 (1996), 119-148.

[28] J. Pöschel, Quasi-periodic solutions for a nonlinear wave equation, Comment. Math. Helv. 71 (1996), 269-296.

[29] J. Pöschel, E. Trubowitz, Inverse spectral theory, Pure and Applied Mathematics 130, Academic Press Inc., 1987.

[30] M. Procesi, X. Xu, Quasi-Töplitz functions in KAM theorem, to appear in SIAM Journal Math. Anal.

$4^{\mathrm{e}}$ SÉRIE - TOME $46-2013-\mathrm{N}^{\circ} 2$ 
[31] W. M. WANG, Supercritical nonlinear Schrödinger equations I: quasi-periodic solutions, preprint arXiv:1007.0156.

[32] C. E. Wayne, Periodic and quasi-periodic solutions of nonlinear wave equations via KAM theory, Comm. Math. Phys. 127 (1990), 479-528.

\author{
Massimiliano BERTI \\ Dipartimento di Matematica e Applicazioni \\ "R. Caccioppoli" \\ Università degli Studi di Napoli Federico II \\ Via Cintia, Monte S. Angelo \\ I-80126 Napoli, Italy \\ E-mail: m.berti@unina.it \\ Luca BiAsCo \\ Dipartimento di Matematica \\ Università di Roma 3 \\ Largo San Leonardo Murialdo \\ I-00146 Roma, Italy \\ E-mail: biasco@mat.uniroma3.it \\ Michela Procesi \\ Dipartimento di Matematica e Applicazioni \\ "R. Caccioppoli" \\ Università degli Studi di Napoli Federico II \\ Via Cintia, Monte S. Angelo \\ I-80126 Napoli, Italy \\ E-mail: michela.procesi@unina.it
}

